COMPUTED TOMOGRAPHIC MEASUREMENT

OF TONGUE VOLUME RELATIVE TO ITS

SURROUNDING SPACE.

\author{
A THESIS \\ SUBMITTED TO THE FACULTY OF GRADUATE STUDIES \\ IN PARTIAL FULFIIIMENT OF THE REQUIREMENTS FOR THE \\ DEGREE OF MASTER OF SCIENCE
DEPARTMENT OF PREVENTIVE DENTAL SCIENCE WINNIPEG, MANITOBA

BY

ELUI G. ROEHM

JULY 1981 


\section{COMPUTED TOMOGRAPHIC MEASUREMENT \\ OF TONGUE VOLUME RELATIVE TO ITS \\ SURROUNDING SPACE}

BY

ELLI G. ROEHM

A thesis submitted to the Faculty of Graduate Studies of the University of Manitoba in partial fulfillment of the requirements of the degree of

MASTER OF SCIENCE

(c) 1981

Permission has been granted to the LIBRARY OF THE UNIVERSITY OF MANITOBA to lend or sell copies of this thesis, to the NATIONAL LIBRARY OF CANADA to microfilm this $\therefore \quad$ thesis and to lend or sell copies of the film, and UNIVERSITY MICROFILMS to publish an abstract of this thesis.

The author reserves other publication rights, and neither the thesis nor extensive extracts from it may be printed or otherwise reproduced without the author's written permission. 


\section{Abstract}

The relationship of tongue size to the space available for it within the oral cavity and oropharynx and how this relationship affects occlusion and facial form are of current clinical interest. This investigation has utilized computed tomography (CT) to assess the three-dimensional relationship of the tongue to its surrounding space. CT is a radiographic technique which produces a series of sequential cross-sectional radiographic images by scanning each tissue slice from multiple angles with a narrow x-ray beam. A series of sequential transverse plane scans or slices, of 5.0 millimeter thickness, were obtained for 32 subjects, commencing at the level of the anterior nasal spine and stopping at the level of the hyoid bone. This ensured that the entire tongue and surrounding space were included in each tongue scan series. An Independent Viewing System (IVS) was used to measure tomographic image areas of the tongue and its surrounding oral space. Tongue and oral volumes were calculated for each subject, using an appropriate mathematical formula, and a ratio tongue volume oral volume was obtained. CT provides the best method at present, in this researcher's opinion, for evaluating accurately the three-dimensional relationship of the complete tongue and its surrounding space.

As the base of the tongue forms the anterior wall of the pharyngeal airway, its postural position is determined principally by factors regulating airway maintenance. The tongue, in its forward 
position, has been considered to be involved in the etiology of anterior open-bite malocclusion. It has been demonstrated that openbite subjects (5) have a mean ratio $\frac{\text { tongue volume }}{\text { oral volume }}(90.91 \pm 0.53)$ which is significantly larger $(p<0.01)$ than that for nonopen-bite subjects $(27)(86.03 \pm 0.69)$. This indicates that the proportion of tongue volume relative to oral volume is greater for open-bite subjects. To accomodate the tongue and not impinge on the airway, it is postulated that the mandible rotates open and the tongue postures forward creating an anterior open-bite.

Cephalometric variables were assessed in this study and the cephalometric results for open-bite versus nonopen-bite subjects were found to be in general agreement with other investigators. 
Dedication

To the birth of my first child, Anita Marie July 1, 1981 


\section{Acknowledgements}

First and foremost, I express my appreciation and thanks to my research supervisor, Dr. A.T. Storey, Professor and Head, Department of Preventive Dental Science, University of Manitoba, for his guidance and support which has seen this project through to completion.

My sincere appreciation to Dr. C.G. Baker, Professor and Chairman, Department of Stomatology, University of Alberta, for originally proposing and initiating this project and arranging for this investigator to analyze part of the data at the Royal Alexandra Hospital, Edmonton.

My special thanks to Dr. D.E. Tubman, Director of CT Scanning, Health Sciences Centre, Winnipeg, for his suggestions in carrying out this research project and for allowing me to utilize freely the necessary facilities.

Thanks to Dr. D.W. MacEwan, Professor and Head, Department of Radiology, Health Sciences Centre, Winnipeg, for approving this research project.

I also express my thanks to Dr. D. Gelskey, Consultant to the Laboratory and X-ray Division of M.H.S.C. (Manitoba Health Services Commission) for his sound guidance and advice in the preparation of this thesis.

My appreciation to Cathy Erickson, Radiological Technologist, CT Area Supervisor, for her careful supervision in conducting CT scans. To Mr. J. Enns, Director of Radiology and Brian Sampson, CT 
Supervisor and Technician, both of the Royal Alexandra Hospital, Edmonton, I express my thanks for the use of the hospital equipment and their kind assistance. Also thanks to Art Young, Senior Technical Representative, Omnimedical, for his part in making it possible to analyze part of the data at the Royal Alexandra Hospital.

My thanks to the Department of Anatomy, University of Manitoba, for supplying the cadaver heads used in this study and to Dr. L. Gibson, Associate Professor of Anatomy, for his assistance with carrying out anatomical dissections and advice on anatomical landmarks.

Also thank you to Dr. K.R. McLachlan, Professor of Civil Engineering, Adjunct Professor to Preventive Dental Science, University of Manitoba, for his assistance in divising an instrument with which to measure palatal vault height.

My appreciation to Dr. F.S. Chebib, Biostatistician, University of Manitoba, for his assistance with statistical analysis of the data and to Nancy Kan for carrying out computer programs.

For preparation of artwork and photography, I thank Mr. A. Domokos, Jerry Kostur and Sharon Stoltz.

I extend my appreciation to Joanne Gilbert for the time, effort, care and patience required in the typing of this manuscript.

To my classmates, Drs. Philip W. Carter and Samuel K. Chiang, heartfelt thanks for their friendship, support and encouragement during this undertaking and throughout the course.

Finally, to my husband Robert, thank you for your support, patience, encouragement and most of all reassurance throughout this 
project.

This study was supported by a grant from the Children's Hospital of Winnipeg Research Foundation. 
Table of contents

Chapter

Page

Abstract

ii

Dedication

iv

Acknowledgements

$\mathrm{V}$

List of Tables $x$

List of Figures xii

List of Appendices xiv

I Introduction 1

II Literature Review 3

III Materials and Methods 22

A) The Experimental Sample 22

B) The Scanning Unit 23

C) Computed Tomograms 33

D) Independent Viewing System 36

E) Reading the EMI Scan 36

F) Area Measurement 45

G) Defining Anatomical Boundaries of the Tongue 53

H) Volume Calculation 61

I) Cadaver Study $\quad 62$

J) Study Model Variables 64

K) Cephalametric Variables 68

L) Measurement Error $\quad 75$

1. Instrument Error $\quad 75$

2. Investigator Error $\quad 75$

i) Pilot Study $1 \quad 75$

ii) Pilot Study 2

iii)Pilot Study $3 \quad 79$

M) Statistical Analysis 83

$\begin{array}{lll}\text { IV Results } & 84\end{array}$

A) Instrument Error $\quad 84$

1. CT Numbers 84

2. Area Measurement 86

3. Cadaver Measurement 90

B) Investigator Error 92

C) Results of Statistical Analysis 93

1. Results of Student's " $t$ " test 94

i. Tongue Volume 94 
ii. Cephalometric Variables

a) Intermaxillary Ht., Lth. and Area, Tongue and Hyoid

b) Cranial Base

c) Maxilla to Cranium

d) Mandible to Cranium

95

e) Mandible to Maxilla

95

f) Dentoalveolar

96

g) Vertical Proportions 97

iii. Model Variables

97

2. Results of Correlations 98

V Discussion

99

$\begin{array}{ll}\text { Tongue Measurement } & 100\end{array}$

Tongue Boundaries 103

Accuracy of Volume Measurement with CT 107

Methods of Measuring Areas on CT scans 109

Tensing or Contraction of the Tongue During Scans 110

Motion Artifact, High - Attenuation Differential

Artifact, Positional Changes of Tongue and Head Between Scans

111

Varying Intersubject Head Positions $\quad 114$

Scan Increments $\quad 115$

$\frac{\text { Tongue Volume }}{\text { Oral Volume }}$ - O. B. Versus N. O. B. 115

Cephalometric Variables - O. B. Versus N. O. B. 117

Model Variables - O. B. Versus N. O. B. 119

Clinical and Research Implications of CT. 120

VI Summary and Conclusions 123

$\begin{array}{ll}\text { Bibliography } & 126\end{array}$

$\begin{array}{ll}\text { Appendices } & 138\end{array}$ 
List of Tables

Table

Page

I CT numbers and linear attenuation coefficients for various body tissues with a magnifying constant of 500 ( $120 \mathrm{kVp}$ - effective $70 \mathrm{keV}$ ).

2 Mean CT number for muscle for each subject as reported by the Independent Viewing System (IVS) .

3 List of Linear and Angular Cephalometric Measurements.

4 Standard deviation of the error, 95 \% maximm error and 99 \% maximum error committed during tongue boundary identification and area measurement on the Independent Viewing System (IVS) in Winnipeg and Edmonton.

$599 \%$ maximm error for volumes calculated from contiguous slices where $n=$ number of slices.

6 Standard deviation of the error, $95 \%$ maximm error and 99 \% maximm error associated with study model variables.

7 Standard deviation of the error, $95 \%$ maximm error and 99 \% maximum error associated with selected angular and linear cephalometric variables.

$8 \quad$ Results of Mixed Analysis of Variance of CT numbers for muscle as reported by the Independent Viewing System (IVS) in Winnipeg and Edmonton for each slice of the same 5 subjects.

9 Means and standard errors obtained for Independent Viewing System (IVS) measured phantom areas and actual phantom areas and the difference.

10 Means and standard errors for calculated cadaver tongue volumes and actual cadaver tongue volumes and the difference.

11 Means, standard errors and level of significance between Group 1 - Overbite $\geq 0$ ( N. O. B. Grp. ) and Group 2 Overbite < 0 ( 0 . B. Grp.) for the variable $\frac{\text { tongue volume }}{\text { oral volume }}$.

12 Means, standard errors and levels of significance between Group I - Overbite $\geq 0$ ( N. O. B. Grp.) and Group 2 overbite $<0$ ( 0 . B. Grp. ) for cephalometric variables. 
13 Means, standard errors and levels of significance between Group 1 - Overbite $\geq 0$ ( N. O. B. Grp. ) and Group 2 Overbite < 0 ( 0 . B. Grp. ) for model variables.

14 Tongue Volume, Oral Volume and Tongue Volume X 100\% Oral Volume

for Group 1 - overbite $\geq 0$ ( N. O. B. Grp. ) and Group 2Overbite $<0$ ( O. B. Grp. ). 
List of Figures

Figure

Page

1 Basic Components of the EMI CT5005 General Diagnostic Scanner.

2 Basic Components of the EMI CT5005 General Diagnostic Scanner.

3 Basic Components of the EMI CT5005 General Diagnostic Scanner.

4 Diagram demonstrates linear translatory movement of the entire frame within the gantry a short distance along a 180 degree arc. The fan shaped $x$-ray beam sweeps through a selected cross-section of the subject's body and the transmitted radiation is counted by the detectors.

5 After the first linear sweep, the entire frame within the gantry rotates 10 degrees and at this new angle commences to translate linearly another short distance along the 180 degree arc. The entire scanning sequence for one scan or slice consists of 18 sweeps, spanning a 180 degree arc.

6 An overall diagranmatic illustration of the EMI CT5005 Scanner.

7 Diagram illustrates a series of transverse plane scans beginning at approximately the level of the anterior nasal spine and continuing inferiorly to the level of the hyoid bone, each scan taken at 5 millimeter intervals. 35

8 Illustration of EMI gray scale with adjustable window width.

9 Standard Phantom Scan. $\quad 48$

10 High Resolution Phantam Scan. 4.8

11 Illustration of Multiplanar Transformations ( MPT ). 51

12 Illustration of a typical tongue scan series for one subject. Slices 01 to 09 are presented with overlay tracings outlining the tongue and surrounding anatomy as indicated by the following legend.

13 Illustration of the instrument devised to measure palatal vault height. 
14 Illustration of positioning on the study model of the instrument used to measure palatal vault height and dial calipers held vertically at the midpalatine raphe in a seat especially prepared for it in the cross-bar. Measurement obtained was reoorded as "total height".

15 Illustration of measurement of the height of the instrument itself on a table top, which was then subtracted from "total height" (as obtained in Figure 14) to obtain "palatal vault height".

16 Illustration of the thirty three cephalometric landmarks used in this study. Their description is found in Appendix V.

17 Illustration of the major cephalometric planes used in this study. Their description is found in Appendix V. 


\section{List of Appendices}

Appendix

I Parental Consent Form

138

II Correlation of CT Number of Homogeneous Materials with Optical Density

III Formula for the Calculation of Volumes 142

IV Definitions of Study Model Measurements 143

V Glossary of Cephalometric Landmarks and Planes 145

VI Description of Linear and Angular Cephalometric Measurements

VII Results of Student's " $t$ " test between Group 1 - Overbite $\geq 0$ ( N. O. B. Grp. ) and Group 2 - Overbite < 0 ( O. B. Grp. ) for the variables of tongue volume, cephalometric oral volume

measurements and model measurements.

VIII Tongue Volume, Oral Volume and Tongue Volume X $100 \%$ for Oral Volume

Group 1 - Overbite $\geq 0$ ( N. O. B. Grp. ) and Group 2 Overbite < 0 ( O. B. Grp. ).

IX Discussion of Cephalometric Variables Between Group 1 Overbite $\geq 0$ ( N. O. B. Grp. ) and Group 2 - Overbite $<0$ ( O. B. Grp. ). 


\section{Introduction}

Forces on the teeth derive from many sources in the dental environment and the stability of the teeth depends on the state of equilibrium of all the forces acting on the teeth i.e. occlusal, eruptive, buccal and lingual forces. It is general knowledge that the surrounding soft tissues are implicated as major contributors to the control of tooth position, of which the tongue has generally been held to play a dominant role. The tongue is a complex and active muscle which fills most of the space within the oral cavity and oropharynx. Abnormal tongue function, for example anterior tongue thrust, has been thought to lead to dental malocclusions. Now, however, more thought has been given to abnormal posturing of the tongue, be it high, low, forward or backward in position, or a combination of these, as an etiologic factor in malocclusion. In addition, it is not known whether aberrations in tongue size relative to oropharyngeal space are present, except in obvious clinical examples, as we have no way of assessing accurately the size of the tongue relative to the space available for the tongue.

Attempts have been made at measuring tongue area (Vig and Cohen, 1974; Cohen and Vig, 1976) and tongue volume (Bandy, 1966; Bandy and Hunter, 1969; Takuda et al., 1980), but none of the techniques used are adequate in this researcher's opinion. The area measurements exclude the third dimension and the volume measurements did not extend far enough posteriorly to include the dorsum of the tongue. 
With the advent of computed tomography (CT), it appears possible to obtain more accurate volume measurements of the tongue than the previous cited studies. CT produces cross sectional radiographic images by scanning sequential tissue slices from multiple angles with a narrow $x$-ray beam. Montgomery et al. (1979) investigated the accuracy of CT for deriving volume measurements of the nasal airway and found it to be possible.

The objectives of this study are:

1) a) to obtain sequential radiographic slices of the tongue of suitable subjects using $\mathrm{CT}, \mathrm{b})$ to use the Independent Viewing System (IVS) to measure tongue areas of each tissue slice and subsequently apply the appropriate mathematical formula to calculate tongue volume, c) to assess the space available for the tongue within the oral cavity and oropharynx, i.e. oral volume, by the same method.

2) a) to execute these tongue scans on a sample of 32 children drawn from the orthodontic patients of the Faculty of Dentistry, University of Manitoba, Winnipeg, Manitoba, b) to establish ratios for tongue size relative to tongue space for open-bite subjects versus nonopenbite subjects, c) to determine correlations of this ratio with dental and craniofacial dimensions. 


\section{Review of the Literature}

There is general agreement that the position of the dentoalveolar structures in an individual is the result usually of the interaction between both inherited skeletal and neuromuscular characteristics and enviromental effects. This concept is based on clinical observations and early contributors were Tomes (1873), Brodie (1952, 1957), Rix (1953), Hovell (1955, 1962), Gwynne-Evans and Tulley (1956) and Ballard (1957). Those who concluded that soft tissue is responsible primarily for dental arch form relative to dental base feel that when the teeth erupt, their crowns are guided into position by the lips, cheeks and tongue around them. At birth the tongue fills the mouth, flowing out between the gum pads to contact the lip and cheek muscles. With the eruption of the teeth, this contact is broken and the muscles can now exert their forces on the teeth, this influence continuing throughout life. Thus, the teeth represent a point of equilibrium although the term equilibrium in no way implies normality.

However other researchers have not been in agreement as to the influence of the oro-facial musculature on the dentoalveolar structures (Brash, 1956; Dixon, 1960; Scott, 1961). These investigators concur that little direct evidence exists as to the effect of normal functional activity of the lips, cheeks and tongue on arch form or the effect of abnormal function in causing such conditions as contracted palates, open-bites and crossbites. More likely, tongue form accommo- 
dates to the dental arches enclosing it, rather than the tongue determining arch form.

There is no simple explanation for the equilibrium that exists, i.e. it cannot be explained by tongue versus lip forces alone. Early investigators assumed that a balance existed between tongue, lip and cheek forces. These observations were largely clinical as there was little evidence quantifying pressures exerted on the teeth. During the 1950's and early $1960^{\prime}$ 's, specially designed strain guage transducers were developed in order to measure muscular forces against the teeth. Major contributors of this information have been Alderisio and Lahr (1953), Alderisio (1954), Winders (1956, 1958), Kydd (1957), Gould and Picton (1962, 1964, 1968), Lear and co-workers (1964, 1965a) and Proffit and co-workers (1964, 1965, 1969, 1970, 1975b). While swallowing and speaking, tongue pressures have always been found to be greater than lip pressures (Proffit and Norton, 1970). These functions act only for a short period of time. It might be more appropriate to consider a longer time period to determine if these forces balance out. Lear and Moorrees (1969) have estimated muscle forces acting on the dental arches over a 24 hour time period as a result of speech, deglutition, mastication and posture. While in two instances there was an overall counterbalance between tongue and cheek activity, on the whole, lingual forces exceeded cheek forces, in 5 of 7 subjects, by a factor of $11 / 2$ to almost 4 . Therefore, other factors have to be considered to explain the equilibrium establishing tooth position. 
Orthodontists feel that the tongue and facial musculature play an important role in alveolar arch form and dental occlusion. Circumstantial evidence for the hypothesis that the teeth lie in a state of equilibrium comes from difficulties encountered in orthodontic treatment, where teeth are moved against muscular forces and subsequently relapse. Certainly other factors are involved apart from oro-facial muscle function. Reitan (1959) has demonstrated that tooth movement results in stretching of the collagenous fibers in both the periodontal ligament (PDL) and gingival tissues and upon contraction of these fibers, relapse occurs. Considering the viscoelastic properties of tissue, Edwards (1968, 1970), Boese (1969), Brain (1969) and Campbell (1975) have suggested that oxytalan fibers contribute to the relapse of orthodontically rotated teeth, reopening of closed extraction spaces and reopening of corrected midline diastemas.

The tongue in particular has been implicated in the etiology of dental malocclusion. Obvious clinical examples are aglossia and macroglossia, i.e. aberrations in the size of the tongue, where collapse or expansion of the dental arches is found. Congenital aglossia is a rare condition and to date 16 cases have been reviewed by Fulford et al. (1956) and Gardiner (1960), to which case reports have been added by Shear (1956), Petterson (1961), Briggs (1965), Magnusson (1972) and Kuroda and Ohyama (1981). Congenital aglossia is associated commonly with other anomalies, often malformation of the digits (Aglossia-Adactylia Syndrome), but there have been exceptions where no anomalies, other than the tongue, were present (Eskew and Shepard, 1949; Magnus- 
son, 1972; Kuroda and Ohyama, 1981). Features described generally include mention of absence of the tongue or only a small lingual elevation present, hypertrophic sublingual ridges, receding mandible with a narrow chin and missing and irregularly positioned teeth. More detail of oral manifestations can be found in descriptions by Eskew and Shepard (1949), Gardiner (1960), Briggs (1965) and Magnusson (1972). All four reports show complete absence of tongue action with contraction of both maxillary and mandibular teeth and bony bases (especially the mandibular) and crowding of teeth. Lingual cusps of upper posterior teeth and buccal cusps of lower posterior teeth show marked attrition, overbite is increased and Frankfort-mandibular plane angle is higher than average. These cases deomonstrate the adaptability of the body tissues and especially the influence of the tongue upon dental arches.

Regarding microglossia, a milder situation than in the case of aglossia would be expected. Small tongues are generally associated with small arches. Harvold (1968) looked at the effect of a small tongue by removing a wedge 2 centimeters in length from the midsection of the tongue of monkeys. After 4 to 6 months, the results were crowding of the teeth and a deeper bite, presumably as a result of contraction of the dental arches.

Macroglossia is another condition orthodontists have seen clinically at one time or another. A striking example may be seen in a case reported by Walker (1962) where lymphangioma of the tongue produced a marked spacing of the anterior teeth. Brodie (1952, 1971), 
Graber (1966) and Hovell (1955, 1962) described the clinical effects of macroglossia which were proclination of the incisors, with spacing and increased arch width. Brodie (1971) states that macroglossia is rarely due to muscle fiber hypertrophy, although he has observed one case which resolved spontaneously (Abelson et al., 1941). More commonly the cause of macroglossia is due to increased lymphoid tissue of the tongue, i.e. lymphangioma. Brodie describes macroglossia as a relative disparity in size of the tongue to the space available for the tongue, that is, situations where the tongue is advanced in development and protrudes between the jaws. This often disappears as Waldeyer's lymphoid ring - lingual, faucial and pharyngeal tonsils atrophies in late childhood and space increases for the tongue or with pubertal growth as jaw growth catches up and increases space available for the tongue.

Different parts and tissues of the body show different growth curves and this has been aptly represented in Scammon's famous 1930 illustration (see Boyd, 1980), where he demonstrates the growth curves of four chief types of tissue (lymphoid, brain and head, general body and reproductive). Proffit and Mason (1975a) have added the growth curves of the tongue and mandible to this illustration. The tongue is assigned a growth curve similar to that of neural tissue whereby most of tongue growth is complete by the age of about 8 years with only a small percentage of growth occurring beyond the age of 8 years. Mandibular growth closely approximates general body growth. The mandible grows more slowly than the tongue and reaches a growth plateau between 
the ages of 8 and 12 years. Remaining growth of the mandible then occurs with the pubertal growth spurt and in postpubertal growth, which may continue up to 20 years and beyond in males (Popovich and Thompson, 1977). Due to this differential between tongue and mandibular growth, the tongue will be positioned relatively high and forward in the oral cavity during the early years and may lead to a diagnosis of macroglossia.

Clinically, several accommodations may occur to a large tongue, whether the tongue is large in a relative or actual sense. If the tongue is large, yet the space for the tongue is adequate or nearly adequate, there may be simply a lowering of the floor of the mouth to accommodate it within the dental arches. On the other hand, a large tongue may rest between the teeth in the buccal segments and impede their eruption, leading to posterior open-bites or resulting in overclosure of the mandible with the impression that the anterior teeth have overerupted. Graber (1963) has suggested that the tongue interferes with eruption of the posterior teeth in Class II Division 2 malocclusions by occupying interocclusal distance and thereby resulting in an increased curve of spee. Indeed, increased interocclusal distance, deep bite and overclosure of the mandible are often seen in Class II Division 2 malocclusions. A large tongue may rest also over the entire occlusal surfaces of the teeth and impede eruption of all the teeth. This will be apparent in intercuspal position, where the mandible will be overclosed or in postural position, where the freeway space will be excessive. 
In other instances, the lower jaw may be protracted or the head elevated to increase space for the tongue; both these compensatory mechanisms tend to increase space within the oropharynx, that is, increase the airway space. An additional adaptation to a large tongue may be that the jaw rotates open and the tongue protracts in order to accommodate the airway, leading to an anterior open-bite (Lowe et al., 1977). In the past, because of the high correlations between anterior open-bite and tongue thrusting, many investigators have considered tongue thrust to be the etiologic factor in the causation and perpetuation of this malocclusion.

Kydd et al. (1963) found that the mean tongue pressure in a horizontal direction against the left maxillary central incisor, in anterior open-bites was at least twice that of the controls. On the other hand,Proffit et al. (1969) found that 4 of 6 children, who had tongue thrust swallows and open-bites, exerted little or no pressure against the anterior transducer while the other 2 subjects showed high anterior pressure. Wallen (1974) monitored vertical pressures during swallowing in a group of normal occlusions and a group of open-bite malocclusions. In the open-bite group, vertical forces opposing eruption during swallowing were less, while in the horizontal plane they were similar to the group with normal occlusion. The work by Lear et al. (1965b), which gives the best data on swallowing frequency, indicates that in a twenty-four hour period, the mean deglutition frequency was about 600 swallows per day. If one considers that one swallow takes one second, then ten minutes a day would account for 
total swallowing activity. In orthodontics, one would never expect tooth movement with a force acting only ten minutes per day. Recently, however, the tonic forward posturing of the tongue has been considered to be involved in the etiology of anterior open-bite (Proffit and Norton, 1970; Proffit and Mason, 1975a; Lowe and Johnston, 1979) and tongue thrust may simply be an adaptation in order to obtain an anterior oral seal during swallowing, i.e. it may be an associative factor instead of a causative one.

The postural positioning of the tongue can be comprehended by describing the anatomical relationships of the area. The tongue is a highly mobile, muscular organ which plays a vital role not only in the digestive functions of suckling, mastication and swallowing, but also in speech. The tongue is suspended by three bilateral attachments: the mandible, the styloid processes of the temporal bone, and the hyoid bone. It is from these bony attachments that the three principle extrinsic muscles of the tongue attach - genioglossus, styloglossus and hyoglossus - and project into the tongue. The intrinsic muscles of the tongue contribute also to its muscular structure. The tip, sides and dorsum of the tongue and part of its inferior surface are free, but its root is connected with the palate by the palatoglossus muscles, with the pharynx through the attachments of the superior constrictor muscles and with the epiglottis by the epiglottic folds. This accounts for the intimate relationship of the tongue with the motor functions of feeding and speaking.

Two other related functions have greater significance perhaps in 
the development of this area. They are 1) accomplishing a patent pharyngeal airway and 2) participating in postural function of the head and neck (Bosma, 1963b). The stabilization of the pharynx in order to maintain the airway precedes stabilization of the head and neck in an upright position and these dual functions influence the position of the structures in the oral cavity, i.e. principally the tongue. The position of the dorsum of the tongue anteroposteriorly will be determined primarily by airway requirements. The position of the mandible itself is important also in the determination of hyoid and tongue position relative to the pharyngeal airway.

This interrelationship can be seen most dramatically in the Pierre-Robin Syndrome, which consists of micrognathia, glossoptosis and cleft palate (Kelly et al., 1978). The respiratory problem is mechanical, being caused by a blocked airway. The tongue is held forward normally by the genioglossus muscle which attaches to the mental spines at the symphysis of the mandible. The genioglossus muscle maintains the tongue under postural control, normally protruding the tongue, overcoming the downward and backward pull of the hyoglossus muscles and the upward and backward pull of the styloglossus muscles. Since the mandible is severely retrognathic in this syndrome, the genioglossus muscle cannot oppose sufficiently these muscles, i.e. there is failure of the genioglossus reflex which normally protrudes the tongue, and thus the tongue falls back into the hypopharynx and blocks the airway. If the infant does survive the first few months of life, this situation will improve with growth of the mandible. 
Lowe and co-workers have substantiated the positive relationship between mandibular rotation, tongue and airway in both man (Lowe et al., 1977) and animals (Lowe, 1978). An increase in genioglossus muscle activity was seen with the inspiration phase of breathing, which Lowe et al. (1977) suggest may be related to the maintenance of a patent airway. Also, on passive opening of the jaw, there was an increase in genioglossus activity of all subjects. This low threshold activity lasted as long as the jaw was held open and increased with increasing amounts of jaw rotation. Again, the increased activity of the genioglossus muscle in response to jaw opening may be related to protection of the airway. As the mandible rotates open, the pharynx decreases in size and unless the base of the tongue is drawn vertically to increase the airway, genioglossus muscle activity will increase and place the base of the tongue in a more anterior position and increase the pharyngeal airway. As has already been indicated, the postural activity of the tongue is considered as a possible etiologic factor in anterior open-bite.

The genioglossus reflex in response to mandibular opening may play a role in the etiology of anterior open-bite. Lowe and Johnston (1979) examined the difference in genioglossus muscle activity in response to mandibular rotations in a group of human subjects consisting of 20 normal occlusions, i.e. no open-bite present, and 9 openbite malocclusions. Base line level of muscle activity was recorded in maximum intercuspation and jaw opening was recorded in millimeters with maximum intercuspation as the starting point. Voluntary jaw 
opening of all subjects resulted in genioglossus muscle activity, however the thresholds differed for the two groups. Seventeen subjects of the control group had jaw openings of greater than 10.0 millimeters before increases in genioglossus muscle activity were observed. The other 3 subjects showed threshold separations of 4.7 , 8.3 and 8.9 millimeters. In contrast, 7 subjects of the anterior open-bite sample presented increased genioglossus muscle activity at less than 3.0 millimeters of jaw separation, the other 2 subjects at 4.2 and 8.7 millimeters of opening. Therefore, minor amounts of jaw opening are able to produce significant increases in genioglossus muscle activity in open-bite subjects. In these same subjects, tongue protrusion could be observed visually with small amounts of jaw opening. Since very light forces acting over a long period of time are able to effect tooth movement (Weinstein, 1967), possibly postural tongue activity could do the same.

The genioglossus reflex produced in man in response to mandibular rotations in experimental situations (Lowe et al.,1977; Lowe and Johnston, 1979), may be related to protection of the airway. This same reflex may be caused by natural causes such as a large tongue, small maxillary and mandibular arches, enlarged or inflamed tonsils and placement of orthodontic appliances such as an acrylic expansion screw appliance. The effect of a large tongue was observed in Harvold's primate experiments (1968), when he placed a piece of plastic in the palatal vault of monkeys. The animals reflexly lowered the mandible and protracted the tongue creating an anterior open-bite and spacing 
of teeth after several months. The tongue was not actually larger, but by decreasing the space available for the tongue, the genioglossus reflex was initiated, as the tongue was now larger in relative terms and had to be accommodated.

The tongue provides an internal form for the dental arch and any aberration in its posture is reflected in the form of the dental arch. If the tongue lies high or low within the oral cavity, the affected arch is overexpanded in relation to the opposing arch, which in turn is narrower due to deficient support. Various forms of buccolingual crossbites are the result of this. Brodie (1950, 1952, 1957) and Graber (1963) observed that in Class III malocclusions, the tongue appears to be at a lower level in the floor of the mouth. The mandibular arch is overexpanded, while the maxillary arch, which does not have the balancing effect of the tongue, is narrow with a high vault. A low position of the tongue may be associated also with mouth breathing as suggested by Baker (1954). This may allow the buccal musculature, especially the buccinator, to act unopposed against the maxilla and may result in bilateral crossbite. Tomes (1873), the first to mention the "V-shaped" contracted upper jaw as a result of enlarged tonsils, found breathing occurred with the mandible open or lowered and the tongue lowered in position also. Ballard (1953) made also an association between low resting position of the tongue, contracted maxilla, long face and high Frankfort-mandibular plane angle, presumably as a result of mouth breathing.

Ricketts (1968) speculated that respiration requirements was one 
factor predisposing to malocclusion through the influence of tongue posturing and possibly even mandibular posturing. He found in a previous study (1954), in patients where the anteroposterior distance of the bony nasopharynx was shallow, the soft palate dropped at a more acute angle from posterior nasal spine (PNS). The tongue thus acquired a forward and downward position in order not to displace the palate up and back and close the nasopharynx. Where the nasopharynx was filled with adenoid tissue, the forward and downward position of the tongue was even more marked. In 1958 (a) Ricketts presented a case where a unilateral crossbite existed in the presence of enlarged tonsils and adenoids. Eight weeks following their removal, the crossbite corrected spontaneously and the tongue became elevated and retracted in position. In another paper (1958b) Ricketts found in a sample of 30 eight year old children, who underwent adenoidectomy, that decreases occurred in the distance from the tongue to the cervical vertebrae, to the base of the skull and to the hard palate. The tongue had retracted in an upward and backward direction several millimeters.

By following a number of patients before and after surgical removal of tonsils and adenoids, Ricketts found several associated characteristics in the presence of large tonsils and adenoids (1968). These include crossbite (functional crossbite and bilateral crossbite with no deflection), open-bite, tongue thrust and mouth breathing. He termed this condition the "respiratory obstruction syndrome". After surgical removal of tonsils and adenoids, the tongue elevated and the 
head rotated downward on the cervical axis. Linder-Aronson's study (1970) supports also the hypothesis that adenoids affect the mode of breathing, which in turn influences the individual dentition and facial skeleton. Children with adenoids and obstructed nose breathing were found to have low tongue posture, a narrow upper arch, crossbite or a tendency to crossbite, retroclination of upper and lower incisors, increased anterior facial height and small sagittal depth of the nasopharynx. A small overbite was found in children with a crossbite or a tendency to crossbite. This brings to mind the so-called "adenoid facies". The initial factor leading to anterior open-bite malocclusion may be related to maintenance of the airway, with subsequent jaw opening and tongue protrusion as demonstrated by Lowe et al. (1977), followed by changes in the dentition and facial skeleton.

Lymphoid tissue size increases rapidly in children preceding puberty and then undergoes spontaneous regression according to Scammon's curve (see Boyd, 1980) for thymus, lymph nodes and intestinal lymphoid masses. Worms et al. (1971) found in their cross-sectional study of 1,408 Navajo children that between the age groups of 7 to 9 years old and 10 to 12 years old, there was an 80 percent spontaneous correction of simple open-bite (i.e. open from canine to canine). Reasons for correction were not given, but one could speculate that involution of tonsils and adenoids may have been a contributing factor in some of the corrections with subsequent repositioning of tongue, mandible and head.

In experiments, where the mode of respiration was switched from 
nasal to oral, Harvold et al. (1973) showed that lower face height increased more in mouth breathing monkeys than control monkeys after 9 and 15 months of oral respiration. This indicates that lowering of the mandible is associated with mouth breathing. The response of the tongue was forward protrusion and pointing which eventually caused morphological changes in the tongue itself. Narrowing of the maxillary arch also occurred. After nasal respiration resumed, the tongue returned to a higher position and a widening of the maxillary dental arch occurred (Harvold and Tomer, in preparation). Linder-Aronson (1975) has found also a significant increase in maxillary arch width within the first year after adenoidectomy, due to elevated tongue position. Recently, Vargervik (1979) noted that during activator treatment, where there was a change from oral to nasal breathing, the mandible as well as the tongue changed to a higher resting position.

However, the tongue may assume a high resting posture, with an overexpanded maxillary arch and a flat palate (Brodie 1952, 1957, 1971; Ballard 1953). Brodie (1952, 1957, 1971) gives an example of a case where the mandibular arch was completely enclosed by the maxillary arch on closure, i.e. Brodie crossbite.

The tongue has a great ability to adapt to its environment. Subtelny and Sakuda (1966) observed cases clinically and with cineradiographs to study lip and tongue function before and after treatment. They found that in most cases studied, the lips and tongue adapted to the new enviroment established by orthodontic treatment. In fact, where tongue thrust was present before correction, it did not exist 
after orthodontic treatment.

Cleall (1965) demonstrated also tongue adaptation in patients who exhibited a tongue thrust pattern during swallowing, where a palatal tongue crib was placed to prevent forward movement of the tongue. Cineradiographs were used to monitor swallowing before, during and after (immediately and 2 months) use of the palatal crib. With the palatal crib in place, the tongue was found to function at a higher and more posterior position. After removal of the crib, the tongue began readapting to its original environment. However, in a few instances during crib wear, there had been a change in incisor position; the tongue adapted to this new environment rather than to the original environment.

This phenomenon does not mean that the tongue will adapt to all changes in environment. Subtelny and Sakuda (1966) observed that the proportionate size of the tongue is a factor which has been overlooked often in diagnosis. Extraction of teeth, in both the upper and lower jaw, may reduce the environment too drastically and the tongue may not be able to accommodate. Protrusive tongue posture may be the result of such treatment. Also, in such cases of misdiagnosis, mechanotherapy may prove difficult, for example, when closing spaces against a tongue which is proportionately large for its environment.

Changes in tongue environment become more important in cases where surgical procedures alter the size of the oral cavity. Takagi et al. (1967) and Wickwire et al. (1972) have shown that after mandibular osteotomies of the ramus there are reflex compensations to pro- 
tect the airway. The hyoid bone is positioned downward and backward, carrying the root of the tongue downward and decreasing the volume of the tongue within the oral cavity. Wickwire et al. (1972) conclude that surgical reduction of tongue size is not necessary, according to the 22 cases of mandibular osteotomies which they followed. Again it is important to consider the proportionate size of the tongue. In some cases it may not be possible for the tongue to adapt as adequately as in the above cases (Wickwire et al., 1972). This may be especially so in the surgical correction of open-bite cases, where forward tongue posture is considered a factor in the etiology of open-bite. Moreover, some authors (Egyedi, 1965; Köle, 1965; Bjuggren et al., 1968) have performed partial glossectomies in open-bite malocclusion with some success, particularly as surgical closure of open-bites have a higher rate of relapse than a mandibular set-back for instance.

Considerable attention has been focused on the role of the tongue and its relation to the etiology of malocclusion. However, attempts to measure the tongue and quantitate its size have been few.

Several investigators (Vig and Cohen, 1974; Cohen and Vig, 1976) using lateral cephalographs, attempted to measure tongue area relative to intermaxillary space. This is only a two dimensional study and a measure of tongue volume would be desirable. One study attempted to measure tongue volume (Bandy, 1966; Bandy and Hunter, 1969) in human subjects, using a system of fluid displacement, similar to a plethysmograph. This study was limited by the extent to which the tongue 
could be inserted into the recording device. Takuda et al. (1980) attempted also to measure tongue volume using an impression technique which they have developed. However they have limited their measurements anterior to the mesial aspect of the first molars. This negates the usefulness of their study, since it is the posterior aspect of the tongue which is more important as a determinant of pharyngeal airway. Hopkin (1967) measured postmortem neonatal tongues and adult tongues for comparison purposes, but did not look at them in relation to the space within which they were contained (i.e. oral volume).

Computed tomography (CT) is a radiographic technique for producing a series of cross sectional images by scanning each slice of tissue from multiple angles with a narrow $x$-ray beam. The transmitted radiation is measured by scintillation detectors and fed into a computer for analysis by a mathematical algorithm. Subsequently, relative linear attenuation coefficients for the various tissue elements in the section are calculated. Finally the reconstruction is displayed as a gray scale tomographic image on a television monitor. The image demonstrates radiographic differences in soft tissues of varying densities, e.g. blood clots, tumors, white matter, etc.

Computed tomography is the end product of years of work by numerous investigators. The mathematical concept was conceived, years before the first commercial CT scanner was built, by Radon (1917), who proved that a two or three dimensional object could be reproduced from an infinite set of all its projections. Oldendorf (1961) and Cormack (1963) understood the concept of CT and built laboratory models but 
neither developed a commercial scanner. Kuhl and Edwards (1968) built a successful scanner for nuclear imaging but not for diagnostic radiology. Hounsfield (1973) was able finally to develop a CT scanner that possessed diagnostic capabilities.

For brief and easily comprehended reviews of the underlying theory of CT, see Brooks and DiChiro (1975) and Marshall (1976). However more extensive technical reviews of the mathematical basis of CT are available in the literature.

Naidich et al. (1978) used computed tomography to visualize marked atrophy of the left half of the tongue due to left hypoglossal nerve palsy. Montgomery et al. (1979) investigated the accuracy of CT scanning for deriving volume measurements of the nasal airway and have found it to be possible. It appears that no one has used CT to measure tongue volume. With the aid of $\mathrm{CT}$ scanning, relatively accurate measurement of tongue volume may be a distinct possibility. 


\section{Materials and Methods}

\section{A) The Experimental Sample:}

The sample population consisted of 32 subjects of both sexes ( 12 males and 20 females), ranging in age from 11 years 3 months to 20 years 3 months, with a mean age of 14 years 4 months and was selected from patients of the Graduate Orthodontic Clinic of the University of Manitoba. Selection was based on (1) willingness of both the parents and the child to participate in the study, after being fully informed as to the purpose of the study, and signing the necessary consent form (Appendix I) and (2) the availability of recent orthodontic records. Records, necessary to this study, included study models, trimmed in centric occlusion and a lateral cephalogram. All records had been taken within three months of the tongue scan study except for the following three subjects - B.Bo., 7.5 months; J.Ki., 4.5 months; and D.Th., 4.5 months. All subjects had no comprehensive orthodontic treatment prior to the study and no subjects were assessed as having obvious tongue anomalies. The distribution of the sample according to Angle's classification of malocclusion was: 14 Class I malocclusions; 13 Class II Division 1 malocclusions; 2 Class II Division 2 malocclusions; and 3 Class III malocclusions. Overjet ranged from -2.91 millimeters to 13.51 millimeters with a mean of 5.45 millimeters. Twenty seven subjects had an overbite ranging from 0.0 millimeters to 7.73 millimeters with a mean of 4.13 millimeters, while five subjects had an open-bite ranging from 0.33 millimeters to 3.69 millimeters with a 
mean of 1.86 millimeters.

\section{B) The Scanning Unit:}

The scanner used in this study was the EMI CT5005 General Diagnostic Scanner located in the Radiology Department at the Health Sciences Centre, Winnipeg, Manitoba. The computed tomography (CT) system includes a patient examination table, scanning gantry containing the $\mathrm{x}$-ray source and detectors, $\mathrm{x}$-ray generator, computer and the operator/viewing console (Figures 1,2 and 3). Some scanners may have other features, but these basic components are essential.

Prior to a scan, the subject is positioned on the examination table and both subject and table are shifted through the aperture of the scanning gantry to the appropriate anatomical level or cross-section where the scan takes place (e.g. head, chest, abdomen). The scanning gantry supports the $\mathrm{x}$-ray tube, the radiation detectors and ancilliary components and contains the mechanical system which provides the motions required for a particular scanning pattern. The movable frame within the gantry of the CT5005 houses an array of thirty sensitive $\mathrm{x}$-ray detectors in alignment with the $\mathrm{x}$-ray tube. The $x$-ray beam is collimated tightly into a fan shape that matches the configuration of the detectors. The subject is placed between the $\mathrm{x}$-ray tube and the detectors such that the $\mathrm{x}$-ray beam passes through a particular cross-section of the subject. Once the scan begins, the $x$-ray tube with its emitting beam and the detectors move in synchrony in a "translate and rotate" fashion about the subject until a 180 
Figure 1. Basic components of the EMI CT5005 General Diagnostic Scanner.

$$
\begin{aligned}
& \text { T - Patient Examination Table } \\
& \text { G - Scanning Gantry ( containing x-ray } \\
& \text { source and detectors) } \\
& \text { a - aperture of the scanning gantry }
\end{aligned}
$$

Figure 2. Basic components of the EMI CT5005 General Diagnostic Scanner.

$$
\begin{aligned}
& \text { C - Computer } \\
& \text { O - Operator/Viewing Console } \\
& \text { M - Magnetic Tape Unit }
\end{aligned}
$$



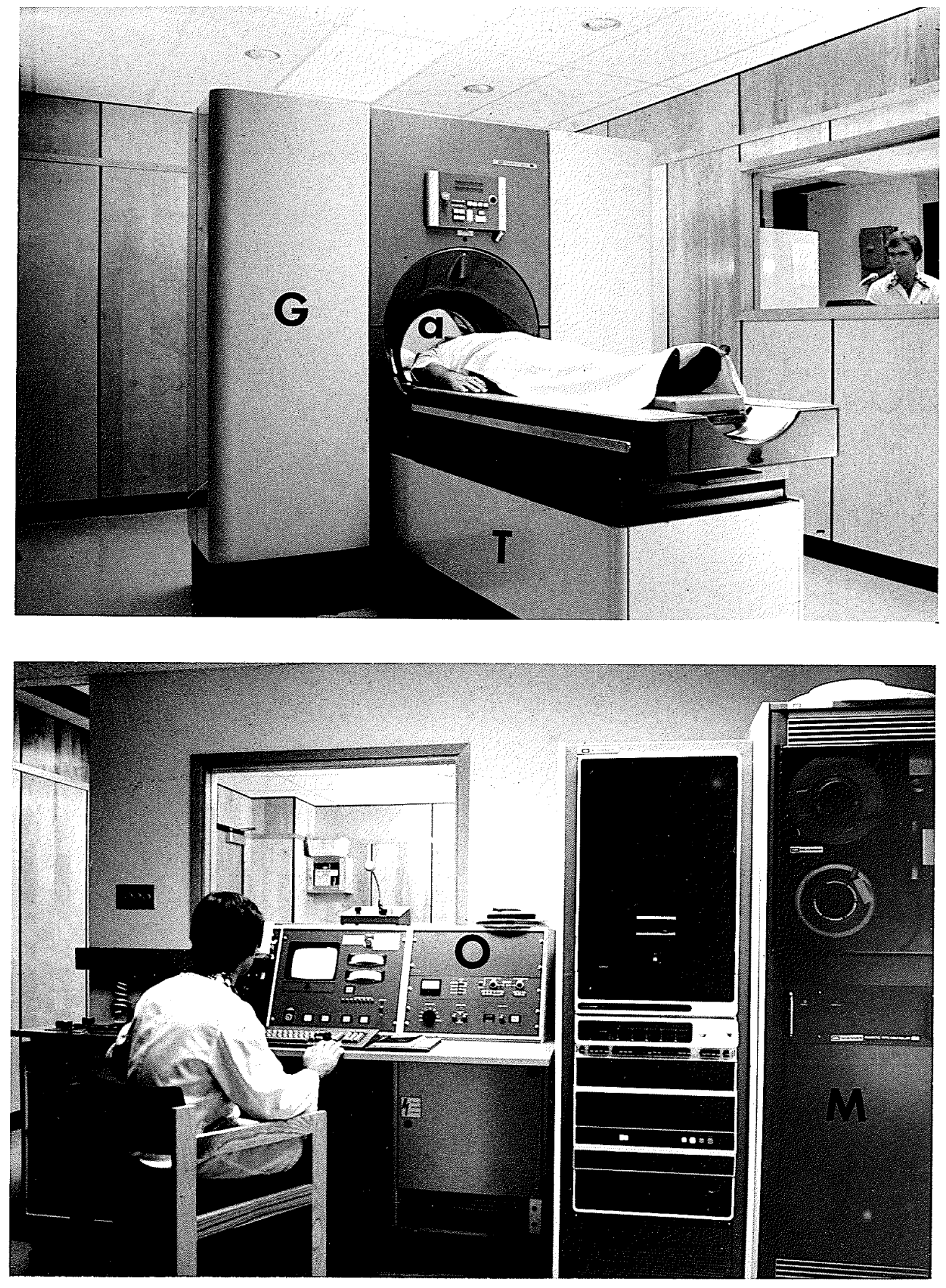


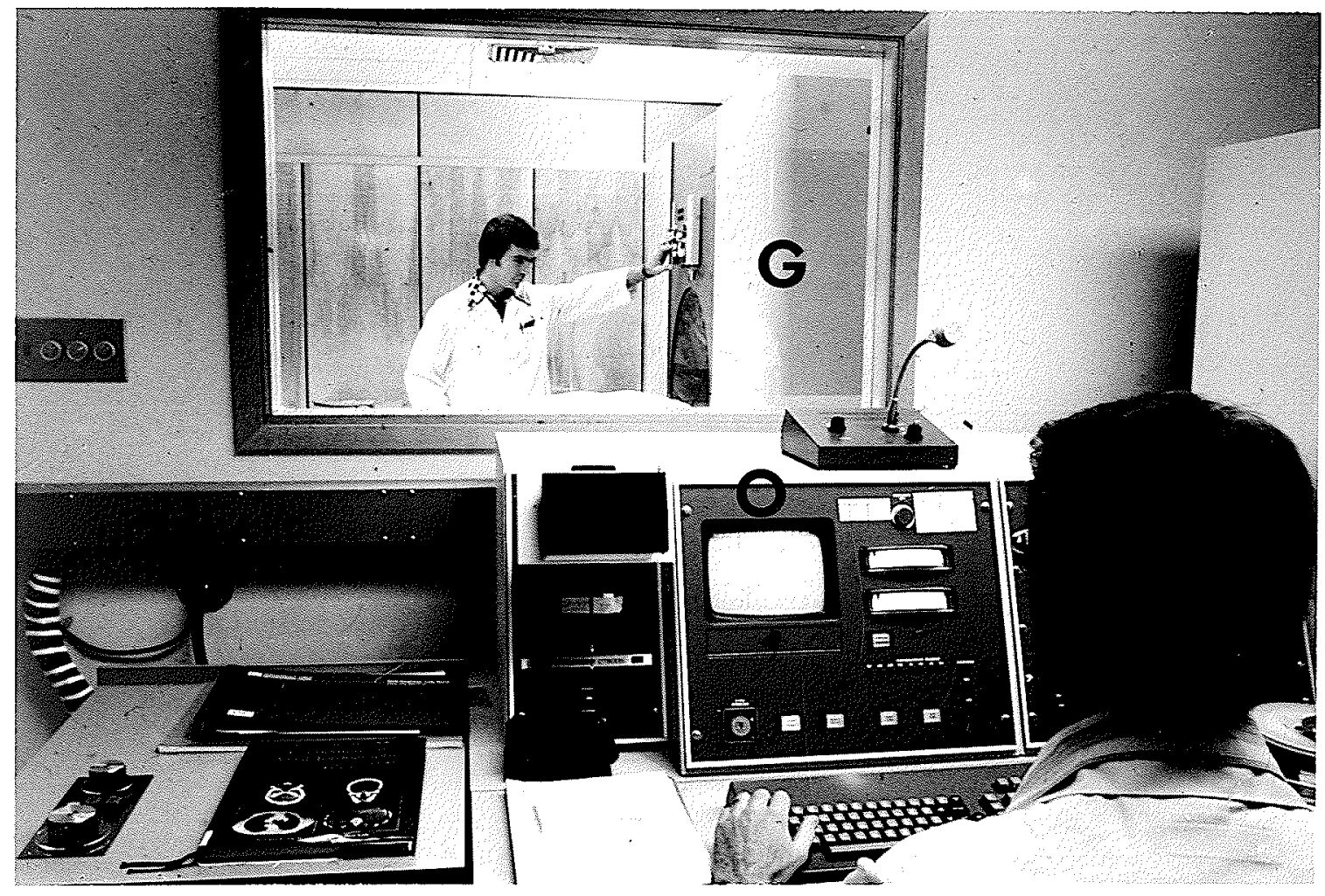

Figure 3. Basic components of the EMI CT5005 General Diagnostic Scanner.

$$
\begin{aligned}
& \text { o - Operator/Viewing Console } \\
& \text { G - Scanning Gantry }
\end{aligned}
$$


degree arc has been completed. This completes one scan.

Therefore, at the beginning of the scan, the frame within the gantry translates linearly a short distance along the 180 degree are and the fan shaped beam sweeps through a selected cross-section of the subject's body (Figure 4). As the x-ray beam penetrates anatomical structures, the intensity of the ray is altered by tissue absorption in the beam's path. The transmitted radiation ( $x$-ray photons) is counted by the detectors. The detectors are made up of scintillation crystals of activated sodium iodide coupled to photomultiplier tubes. When a scintillation crystal absorbs an $\mathrm{x}$-ray photon, the interaction produces light (a scintillation), which is proportional to the energy of the $x$-ray photon. Light from the scintillation crystal strikes the photoemissive surface of the cathode within the photomultiplier tube and causes the surface to emit electrons. The electrons, in numbers proportional to the intensity of the light, are multiplied in the photomultiplier tube in a step-wise fashion to produce an amplified electrical signal. These electrical signals are received and stored by the computer during each linear sweep of the $\mathrm{x}$-ray beam. Therefore the detector does not form the image but measures the number and energy of the transmitted photons. More than 18,000 readings are obtained and stored by the computer each time the $\mathrm{x}$-ray beam makes $\mathrm{a}$ single linear sweep. The $x$-ray beam intensity is monitored continuously by a reference detector. At the end of each linear sweep, calibration readings are recorded and used to compensate for any drift that may have occurred in detector response during the sweep. When 


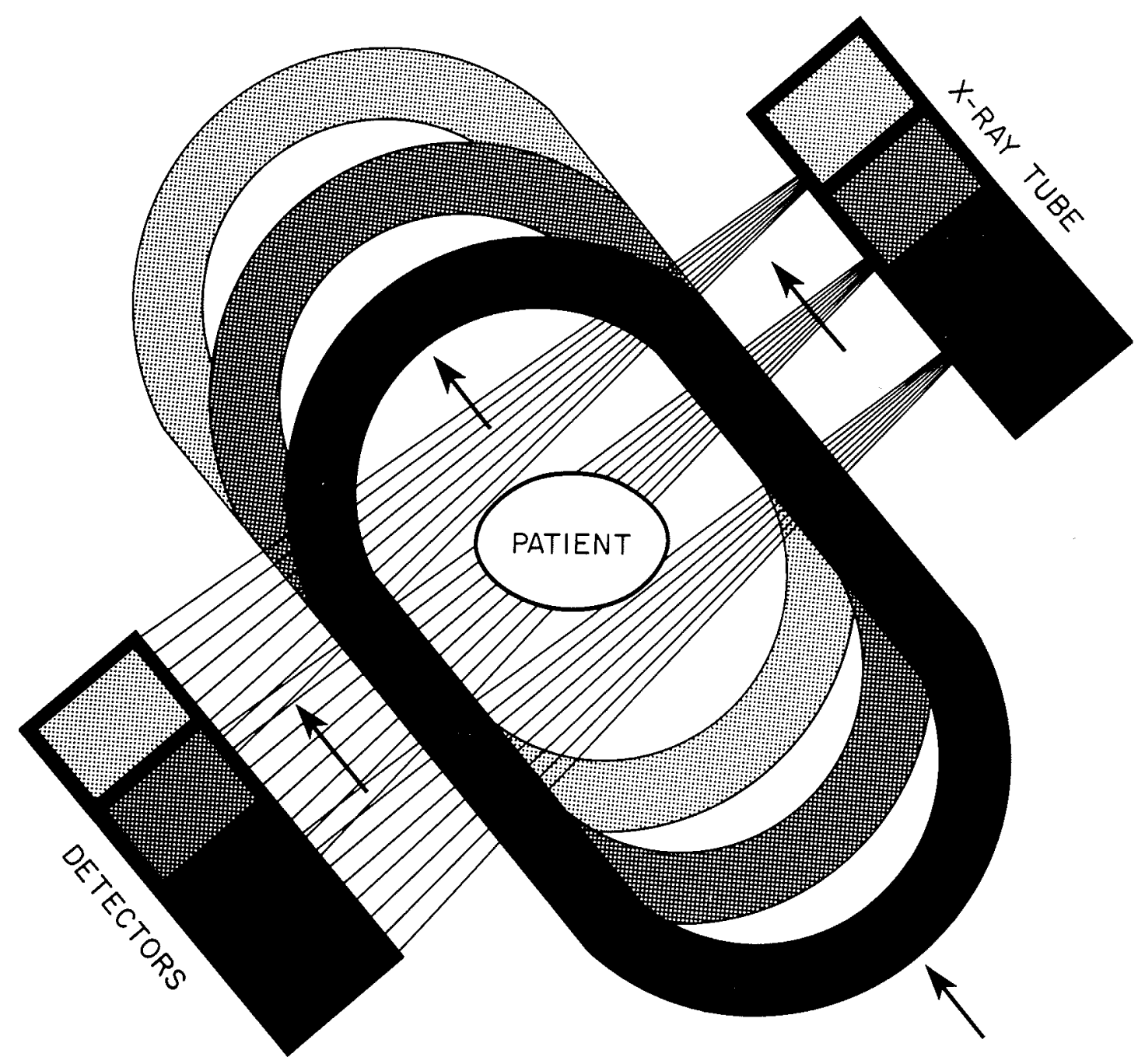

Figure 4. Diagram demonstrates linear translatory movement of the entire frame within the gantry a short distance along a 180 degree arc. The fan shaped $\mathrm{x}$-ray beam sweeps through a selected cross-section of the subject's body and the transmitted radiation is counted by the detectors. 
the first linear sweep is completed, the entire frame within the gantry rotates 10 degrees (Figure 5). At this new angle, it will again translate linearly a short distance along the 180 degree arc. As the $x$-ray beam passes through the same cross-section of the subject's body during this second linear sweep, another 18,000 readings are collected and stored by the computer at the new angle.

Linear translation, detection, information storage, recalibration and rotation continue until the scanning frame has rotated 180 degrees about the subject. One scan, also called one slice, through a specific cross-section of the subject's body, is then complete. The scanning time is the amount of time required to complete one 180 degree cycle. After one scan has been completed, the examination table shifts a selected interval (e.g. 5 millimeters) and moves either into or out of the aperture of the gantry; the next scan begins at this new cross-section of the subject's body.

To produce a CT image, the computer processes over 300,000 discrete bits of information, representing absorption values taken from eighteen different angles. A series of computer calculations estimates the $x$-ray absorption value (linear attenuation coefficient) for each specific pixel point within the scanned section. In computed tomography, a cross-sectional layer of the body is divided hypothetically into thousands of tiny blocks called voxels (volume elements). If the cross-sectional layer is 5 millimeters thick, then the voxel will be a 5 millimeter column. Each voxel is represented by a flat square surface called a pixel (picture element) in the CT image. 
Figure 5. After the first linear sweep, the entire frame within the gantry rotates 10 degrees and at this new angle commences to translate linearly another short distance along the 180 arc. The entire scanning sequence for one scan or slice consists of 18 sweeps, spanning a 180 degree arc. 


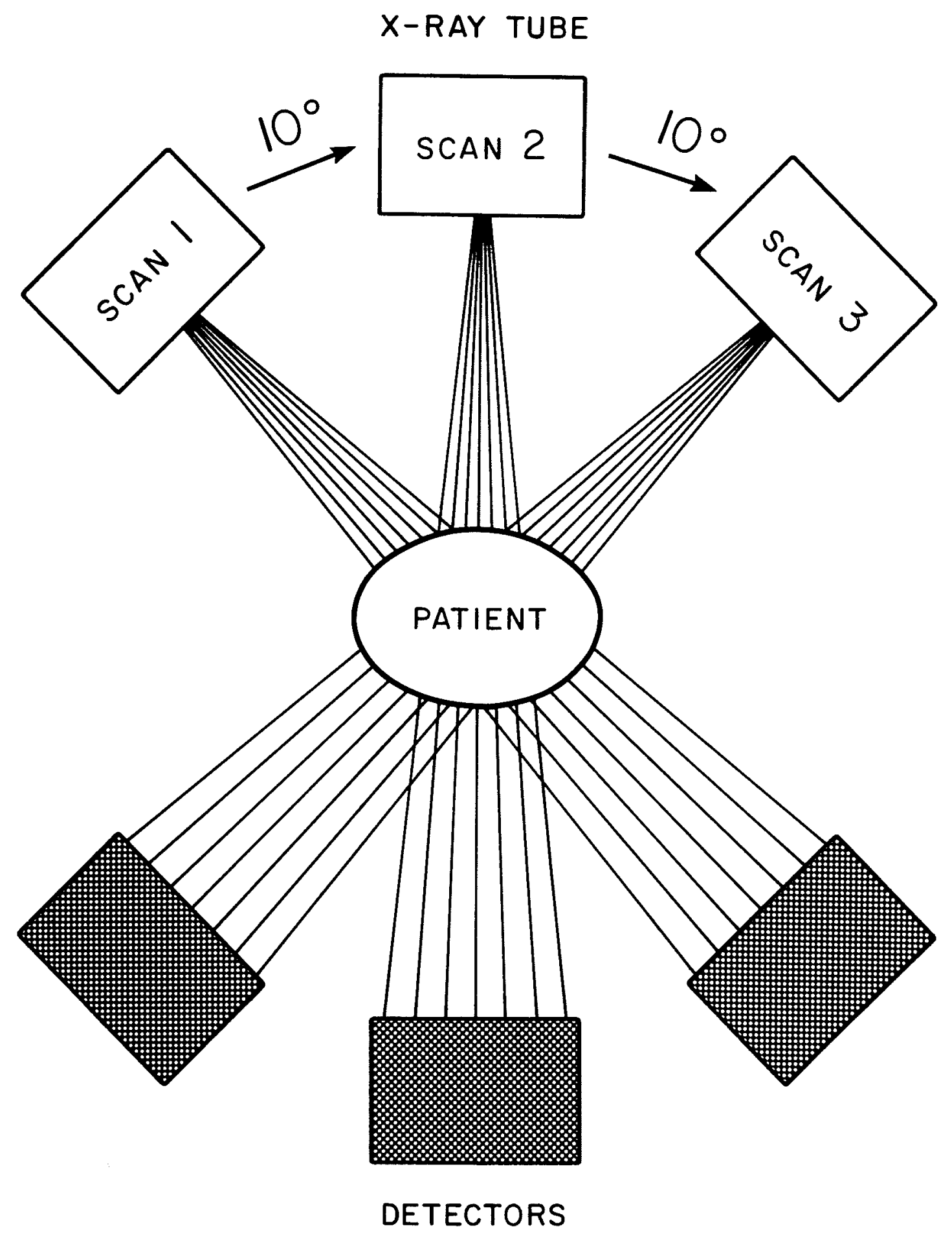


Therefore, the image is made up of thousands of tiny pixels, each representing a specific tiny cross-sectional volume of the subject. Each pixel is assigned by the computer a linear attenuation coefficient which is the average of the linear attenuation coefficients for all the tissue elements within that pixel. The matrix size refers to the number of pixel points in a picture matrix, for example; 25,600 in a $160 \times 160$ matrix or 102,400 in a $320 \times 320$ matrix.

The linear attenuation coefficients for each pixel within the scanned section are stored digitally on a magnetic disc in the computer and represent a mathematical digital "picture" of the subject's anatomy. The data is available for video display as an image analog to the digital distribution. This data may be inspected visually on the viewing console or as a hardcopy printout of digital values. Images displayed on the video screen may also be photographed using photographic film. Computer discs are not used for long term information storage. This function is delegated to magnetic tape or "floppy" discs which can store a few images on each. Figure 6 illustrates diagrammatically the components of the EMI CT5005 Scanner.

In essence, computed tomography (CT) produces a visual reconstruction, called a CT image, tomographic image or tomogram, by computer of a tomographic plane of a subject. In medical applications, this plane represents approximately a one centimeter slice through some cross-section of human anatomy, but the thickness can be varied. Conventional radiographic tomography, as compared to computed tomography (CT), is a special $x$-ray technique which blurs out the shadow of 


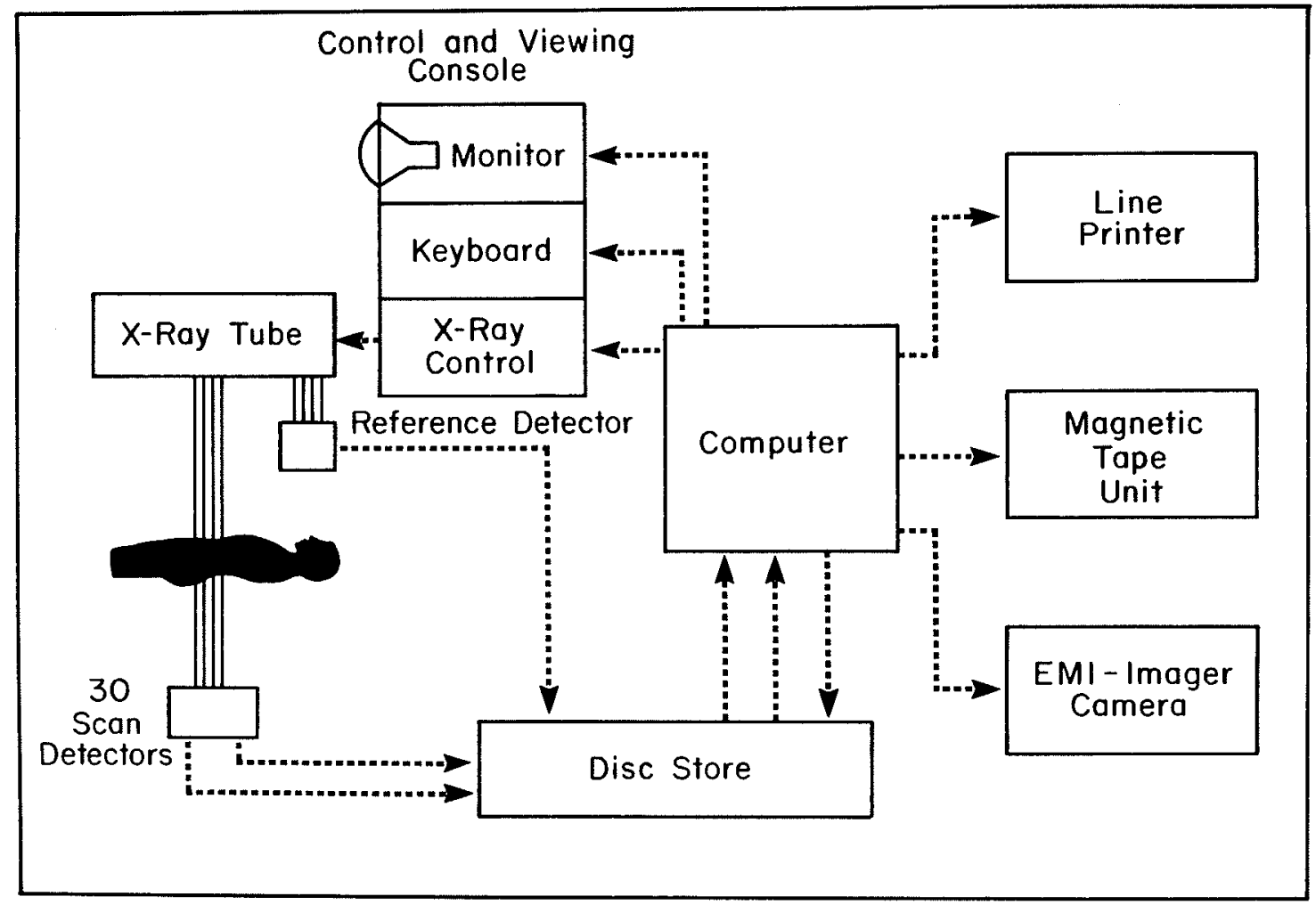

Figure 6. An overall diagrammatic illustration of the EMI CT5005 Scanner. 
superimposed structures from layers of the body either above or below the plane of interest. This method demonstrates the structures being examined in the selected plane of interest without superimposed densities. The $x$-ray tube and $x$-ray film holder are joined by a rigid rod which oscillates creating a fixed fulcrum. When the tube moves in one direction, the film moves simultaneously in the opposite direction, while the fulcrum is the only point in the system that remains stationary. The plane of interest is located at the level of the fulcrum, called the focal trough, and structures in this plane remain in focus. Structures located above and below the focal plane are blurred. Therefore, in conventional radiographic tomography a large portion of the available information is lost due to superimposed images from above and below the plane of interest. Hypocycloidal and transpiral tomography have enhanced the clarity of the slice. In order for any one structure to be seen in the selected plane, it must stand out clearly from the structures above and below it. Conventional tomography provides relatively low contrast images with relatively little quantitative information about the $\mathrm{x}$-ray absorption properties of the structures imaged. In contrast, $\mathrm{CT}$ confines the radiation to the plane of interest, minimizes blurring, and permits visualization of small variations in tissue density, not possible by other radiographic techniques. It is also in no way limited by the requirements of $x-$ ray film. 


\section{C) Computed Tomograms:}

The subjects were instructed to lie supine on the examination table and were provided with a lead apron to protect the thyroid gland and gonads. The head was positioned in the head support by this investigator such that the Frankfort Plane was approximately perpendicular to the floor. The head support is shaped in a semi-circular fashion to accept the back of the head, while the vertex of the head is positioned firmly against the top of the head holder. The examination table was then shifted into the circular aperture of the gantry to the appropriate level of the head where the first scan was to begin. A series of slow, high resolution scans or slices (matrix size $320 \times 320^{*}$, field size 12 centimeters**) were performed for each individual, which last 73 seconds for each scan or slice. According to recent work by McCullough and Payne (1978), patient dosage with the EMI CT5005, at $140 \mathrm{Kvp,} 28 \mathrm{~mA}$ and scanning time of 73 seconds is 1.9-9.6 rads (radiation absorbed dose) per scan. The EMI CT5005 at the Health Sciences Centre incorporates a dose reduction collimator which decreases the dosage by 45 percent, further reducing the dosage range to $1 \cdot 0-5.3$ rads per scan. The exposure to the centre of the head increases 2.4 times in going from a one to a five slice study

* Matrix size is the number of pixel points in a picture matrix e. 9. , $160 \times 160$ matrix $(25,600$ pixels), $320 \times 320$ matrix $(102,400$ pixels).

** Field size refers to the outside dimensions of the CT slice and dictates the maximum size of the anatomic part that can be examined. 
using an EMI CT5000 Scanner set at 70 seconds (MoCullough and Payne, 1976). This amount of radiation can be compared to a dental full mouth survey, consisting of sixteen periapical films and four bitewing films. According to measurements done in the Department of Radiology at the Faculty of Dentistry, University of Manitoba, skin entrance dose for a full mouth survey taken at $75 \mathrm{Kvp}$ and $15 \mathrm{~mA}$ is $4-6$ rads*.

A series of transverse plane scans of the tongue were taken at intervals of 5.0 millimeters, beginning at the level of the anterior nasal spine and stopping at the level of the hyoid bone, as viewed on the video screen monitor (Figure 7 ). The total number of sequential scans or slices obtained for each subject will be referred to as the "tongue scan series" or "scan series" for that subject.

Prior to the study, each subject was instructed to keep his or her teeth together in intercuspal position (centric occlusion), to continue regular breathing during each scan but not to swallow or move the tongue tip. To reinforce this, several minutes were spent with each subject prior to the study, maintaining the tongue still for 73 seconds and holding it in a stable position. Subjects were allowed to swallow between scans and when they heard the next scan begin, to again maintain the tongue in a stable position for 73 seconds. Most subjects learned to accomplish this relatively easily.

* As measured on Electronics Instruments Limited Model 370 x-ray dosimeter. 


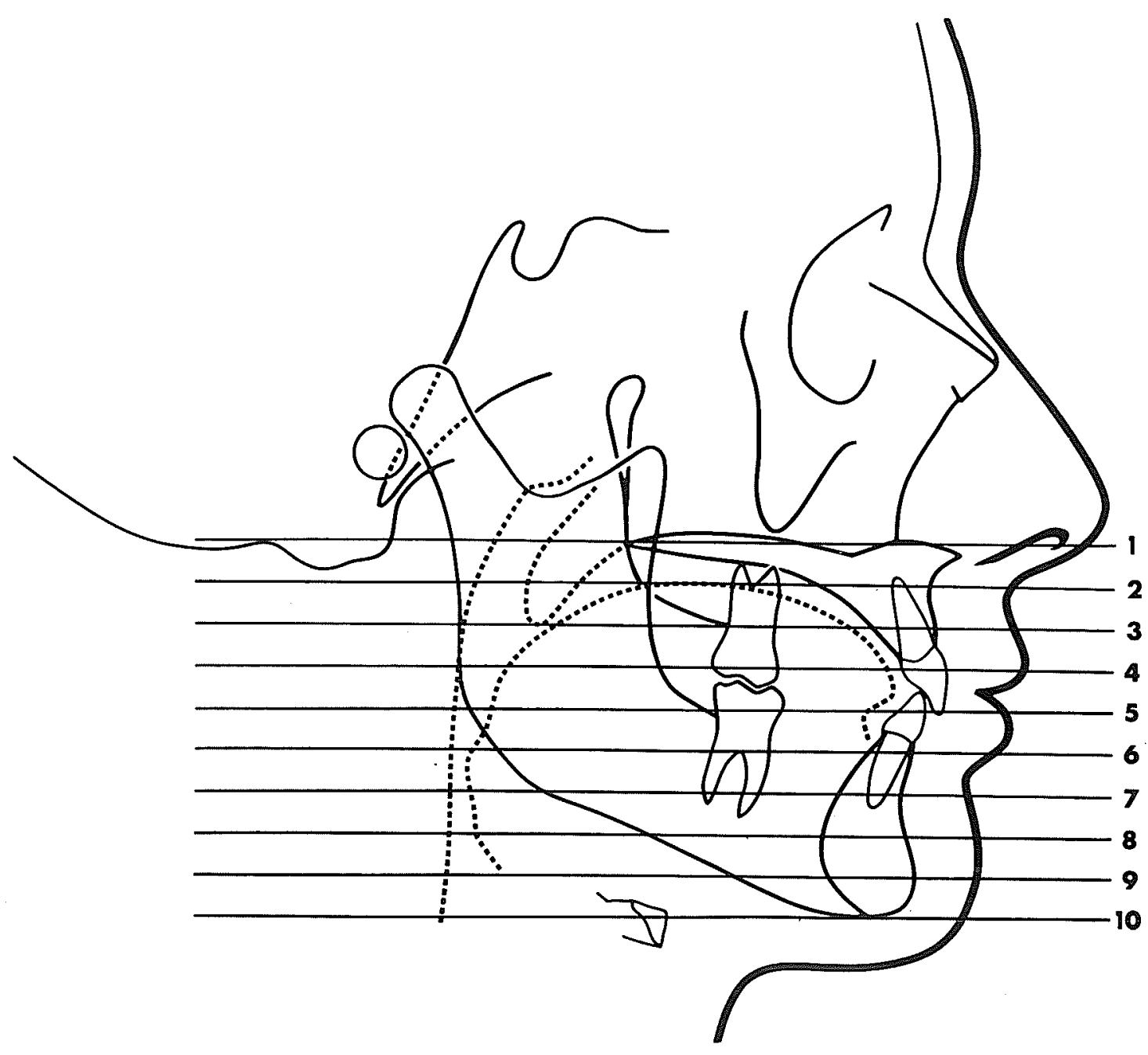

Figure 7. Diagram illustrates a series of transverse plane scans beginning at approximately the level of the anterior nasal spine and continuing inferiorly to the level of the hyoid bone, each scan taken at 5 millimeter intervals. 


\section{D) Independent Viewing System:}

The Independent Viewing System (IVS) is used independently of the main EMI scanner. It consists of a viewing console and keyboard (similar to that of the EMI scanner), a computer and magnetic tape unit. Some Independent Viewing Systems, rather than having a magnetic tape unit, have a floppy disc unit. A series of software programs extend diagnostic capabilities and permit manipulation of the EMI scan data to obtain diagnostic information concerning a select image area. The capabilities of the IVS of interest to this study are: CT image viewing and manipulation; calculating areas of a particular region of interest (ROI); identifying CT numbers of a particular region of interest; and reconstructing transverse images in both a coronal and sagittal plane. These capabilities will be discussed in more detail later.

E) Reading the EMI Scan:

CT imaging is based on the fact that different anatomical tissues absorb radiation to varying degrees. The absorption characteristics are detected by the scanner and analyzed by the computer which calculates a relative linear attenuation coefficient (u) for each picture element (pixel). This coefficient is normalized to a reference material (water), magnified to a larger whole number by a scaling factor and reported as a new number called a CT number. 


$$
\mathrm{CT} \text { number }=\mathrm{K} \mu_{\underline{p}}-\mu_{\mathrm{W}}
$$

where $\mathrm{K}$ = scaling factor

$$
\begin{aligned}
& \mu_{p}=\text { linear attenuation coefficient of pixel in question } \\
& \mu_{W}=\text { linear attenuation coefficient of water. }
\end{aligned}
$$

Water, for most scanners has a fixed CT number equal to zero and the scaling factor for the EMI CT5005 is 500. Dense bone has a CT number of +500 and air has -500 . All other tissues are intermediate in value as shown in Table 1 (Christensen et al., 1978).

Although CT images can be displayed as a hardcopy computer printout* of CT numbers, in this study, each slice from each tongue scan series was viewed as a gray scale image on a cathode ray tube monitor. At full scale, dense bone with a CT number of +500 is assigned a gray scale level of white and air, at -500 , the level of black. Most tissues of interest to radiologists are contained in the range of -10 to +50 EMI units (McCullough, 1977). Console controls on both the EMI CT5005 and the Independent Viewing System (IVS) allow one to adjust parameters to achieve the most useful display of absorption values. This is done usually by controlling the window level and the window width.

The window level is adjusted according to the material being viewed. For instance the window level may be set at a CT number of +15 for viewing white matter (Christensen et al., 1978). The tongue, since it is made up largely of muscle, should be viewed at a window level set for muscle. Only one article to date has reported an

* The computer printout is time consuming and is used primarily to evaluate scanner performance and not as an imaging method. 
Table 1. CT numbers and linear attenuation coefficients for various body tissues with a magnifying constant of 500 (120 kVp effective $70 \mathrm{keV})$.

\begin{tabular}{lcc}
\hline Absorber & CT Number & Linear Atten. Coef. $\left(\mathrm{cm}^{-1}\right)^{*}$ \\
\hline Bone (dense) & +500 & 0.380 \\
Intracranial & +25 & 0.200 \\
$\quad$ soft & to & to \\
$\quad$ tissues & +5 & 0.192 \\
Water & 0 & 0.190 \\
Fat & -50 & 0.171 \\
Air & -500 & 0 \\
\end{tabular}

* These linear attenuation coefficients were calculated from the CT numbers, except for water, which was taken to be 0.190. (Taken from Christensen, Curry and Dowdey, 1978). 
average CT number for muscle of 33.5 which was established in monkeys (Phelps et al., 1975). Since the CT number will represent an average attenuation coefficient for all the components of a tissue, the CT number for muscle could vary from subject to subject depending on the relative content of muscle, fat and connective tissue. Therefore, an average window level was established for muscle for each particular subject. The IVS was used to accomplish this. In at least five slices of each subject's tongue scan series, a homogeneous mass of muscle (usually the tongue, medial pterygoid or masseter), relatively free from artifact due to amalgam restorations or teeth, was selected. A two to three square centimeter area was outlined and a mean CT number and standard deviation assigned to that area by the IVS. The five slices which showed the smallest standard deviation were used to establish an overall mean CT number for muscle for each subject and the window level set accordingly. These mean $\mathrm{CT}$ numbers are reported in Table 2 .

Once the interpreter selected the window level, the window width was also adjusted. The window width can be adjusted in various increments from full scale to a single unit. The size and number of increments are determined by the manufacturer and on both the EMI CT5005 and IVS used in this study, increments of window width available are $400,200,100,75,50,40,30$, and 20,400 being full scale or maximum and 20 the minimum window width. The viewer can, in effect, create a window that reveals the critical information and excludes all else. For instance, if the window level is set at 0 and the window width at 
Table 2. Mean CT number for muscle for each subject as reported by the Independent Viewing System (IVS).

\begin{tabular}{lrlc}
\hline Subject & CI Number & Subject & CT Number \\
\hline C. Ad. & 22.1 & & \\
B. Al. & 21.7 & K. Mat. & 13.7 \\
B. Bo. & 7.9 & D. May. & 10.8 \\
C. Ca. & 37.6 & N. Maz. & 3.7 \\
D. Ch. & 7.5 & T. Mo. & 28.2 \\
M. De. & 18.9 & C. Pi. & 27.9 \\
D. Fu. & 18.7 & C. Po. & 27.2 \\
D. Ge. & 34.4 & T. Qu. & 37.2 \\
S. Gr. & 28.5 & R. Ri. & 37.1 \\
R. Had. & 27.6 & B. Ro. & 24.6 \\
M. Han. & 25.8 & D. Sc. & 24.8 \\
Y. He. & 24.8 & C. Ter. & 39.5 \\
W. Jac. & 45.5 & C. Tes. & 3.6 \\
L. Jan. & 39.5 & D. Th. & 41.3 \\
J. Ki. & 48.9 & M. To. & 12.6 \\
P. Li. & 40.7 & D. Wo. & 34.9 \\
& & L. Yo. & 47.4 \\
\hline
\end{tabular}


100, all CT numbers between -50 and +50 will appear as shades of gray, while all numbers less than -50 will be displayed as black, all those more than +50 as white (Figure 8 ). The range of CT numbers encompassed by one gray level increases as the window is made wider and decreases as it is made narrower. For diagnostic purposes it is desirable that the full range of gray shades in the image be made to represent a narrow range of absorption values. This reveals very subtle changes in tissue structure. Use of full scale would obscure subtle changes. Although adjusting window level and window width in no way alters the basic information stored in the display memory, it does change the appearance of the image by changing the edges of a particular structure. For this study, once the window level was determined and set, the window width was set at full scale (400) for area computations to be described later. It was felt this would allow maximum visualization and maximum area computation of the tongue and oral spaces and would not change the appearance of the boundaries of the tongue, thereby possibly excluding some tongue. However, while determining boundaries of the tongue for a particular slice, prior to area measurement, the window width was decreased in order to increase the contrast and aid in defining the boundaries. Then all area measurements were made while viewing the image at full scale.

Occasionally, boundaries for the tongue or boundaries of the space within which the tongue lies, show a transitional zone of image density; for example, between a less dense area (air) and a more dense area (soft tissue). That is, a soft tissue boundary does not always 
Figure 8. Illustration of EMI gray scale with adjustable window width.

On the left-hand side, window level is set at 0 and window width at full scale ( 400 on the EMI CT5005) which encompasses the full range of CT numbers from -500 to +500 . In the center, window width has been decreased to 100 with the window level still set at 0 . The full range of the gray scale now represents a narrower range of CT numbers allowing subtle distinctions between tissues to appear, as exemplified by those listed on the right-hand side.

( Adapted from Christensen, Curry, III and Dowdey, 1978 ) 

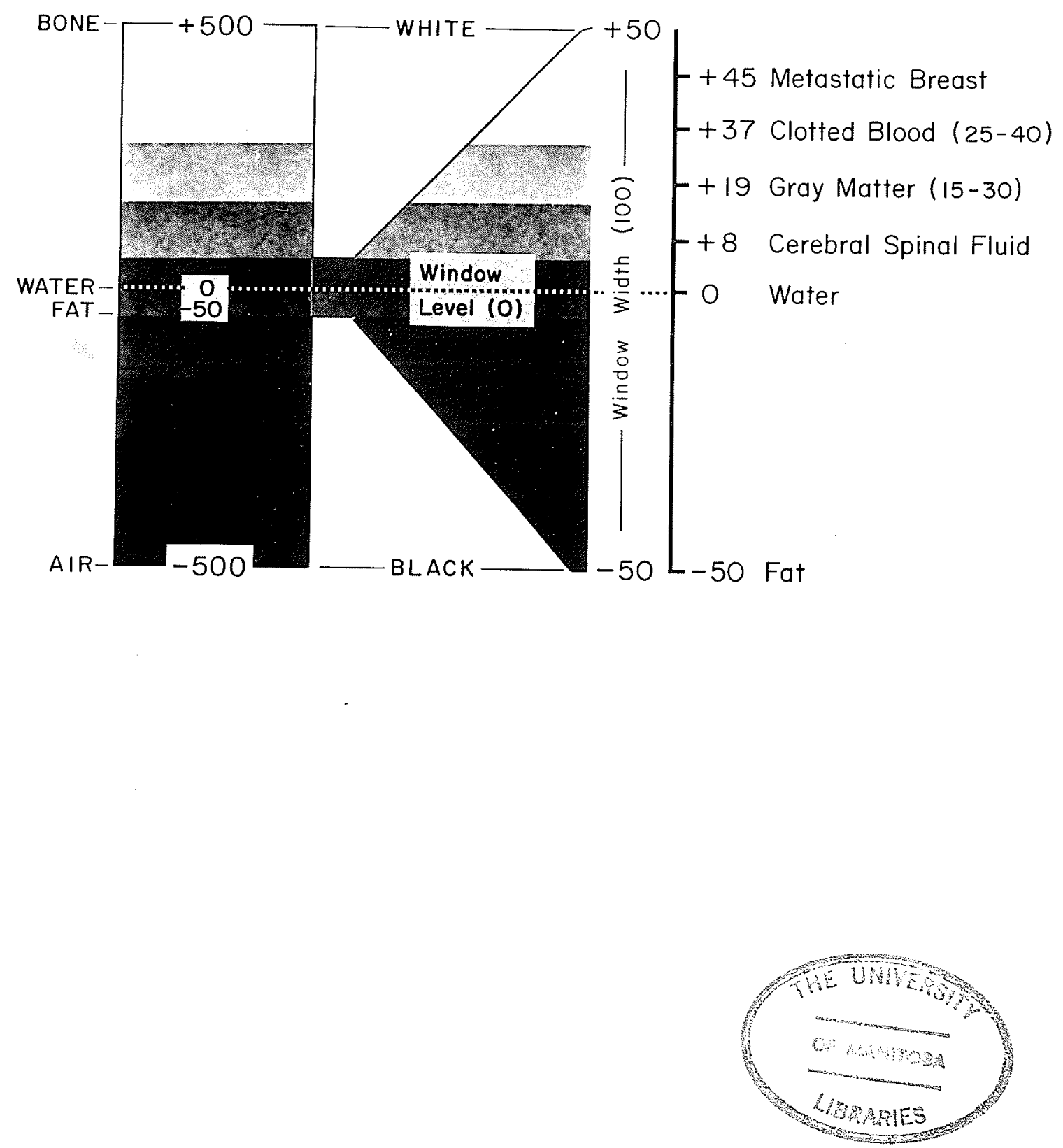
end abruptly next to a region of air and instead, a region of gradual transition will be seen from soft tissue to air. Therefore, boundaries were outlined in the middle of such transition zones in order to take into account "partial volume averaging". The calculated linear attenuation coefficient for a pixel is a weighted average of all the anatomical elements in a pixel. For those pixels in transition zones between areas of different densities (soft tissue - air), the average will be weighted towards the substance with the higher linear attenuation coefficient. This effect is called "partial volume averaging".

The EMI CT5005 Scanner is subject to scan to scan variation or drift, which may compromise the use of CT numbers for differential diagnosis, if it is excessive. Such variations can be monitored by checking the drift in the CT number of water with time. Variations from scan to scan on a day to day basis are part of the quality assurance (consistent performance) aspect of CT scanning. As part of a quality control program, the CT scanner used in this study is monitored to ensure consistency of the mean CT value for a given water bath for a phantom scan. That is, each morning, prior to the day's scanning, a phantom scan is generated and the mean and standard deviation for the water bath checked and logged. These mean CT values for water were recorded, when available, for each day that subject and cadaver scans were performed, and for two days before and after the same scans. There was a period of two weeks in June 1980, when $x-r a y$ technicians were on strike and quality assurance was not done. However, this invesigator was allowed to carry out some subject scans for 
research purposes and the scanner was used for this and any emergency scans during that period. Therefore quality control mean values were available only for the days before and after this particular period. This day to day variation for the CT value of water will be discussed further in the "Results" section (page 84). A check to establish the sensitivity of the CT system (i.e. CT number) in detecting differences in inherent constrast (chemical composition) was carried out using phantom scans and is described in Appendix II.

Since the IVS was used to calculate slice areas of subject tongue scan series, it was desirable to know that the CT number of water of the CT image as viewed on the IVS video screen was not different from the CT number of water as computed and stored in the original image by the EMI CT5005. In order to check this, five phantom scans, taken at different times, were selected and the region of interest (ROI) capability of the IVS utilized to obtain the mean CT number for a water bath. This was compared to the mean CT number for water obtained from quality control readings and will be discussed further under "Results" (page 85 ).

The above was checked on phantom scans using the IVS in Winnipeg only. In order to check that the IVS in Edmonton reported CT numbers that were not different from those reported in Winnipeg, the CT numbers of muscle for 5 subjects were compared. Approximately the same area of the same muscle of the same five slices of the tongue scan series were compared for these 5 subjects. As discussed previously, the CT number will vary from slice to slice within the same subject 
since muscle is not a homogeneous tissue and the CT number is a result of the average attenuation coefficients of all the components of that tissue. Also there is variability between subjects since muscle varies in composition from subject to subject. A mixed analysis of variance was employed in order to measure the variability in CT number of muscle due to each of the following:

1) variation among subjects

2) variation among slices within subjects

3) variation according to location (Winnipeg versus Edmonton).

These findings will be discussed in the section "Results" (page 85 ).

F) Area Measurement:

The tongue scan series data for each subject were stored on both magnetic tape and floppy dise for further study. While photographs of each scan series were taken for each subject, they were not used for area measurement, but only to study the anatomy, to check for any obvious position changes of the head or tongue and to check for any repeated slices. The Independent Viewing System (IVS) and its series of software programs were used to study the data on the tapes. One tape containing tongue scan series for just over one-half of the subjects (17 subjects) proved to be unreadable due to read errors and artifacts present on the scans. This was due to technical problems with the magnetic tape unit at the time some of the scans were performed. Fortunately, the data for these 17 subjects were also stored on floppy discs and not lost. Since the IVS in Winnipeg is connected 
to a magnetic tape unit rather than a floppy disc unit, the floppies could not be studied in Winnipeg. Therefore it was arranged that part of the subject data be analyzed at the Royal Alexandra Hospital, Edmonton, Alberta by this investigator, where the IVS was connected to a floppy disc unit.

Therefore the IVS accepts either magnetic tape or floppy disc, depending on location, transfers CT images off the tape or floppy into the region of interest (ROI) software program and allows area measurement of the EMI scan data. Areas for each slice of tongue scan series were measured directly from the image on the video screen. A tracker ball was used to outline the appropriate area, in a manner similar to a planimeter. The area was calculated by the computer and then displayed on the screen. In Winnipeg, two slices were selected at random from each tongue scan series for 15 subjects and tongue areas measured twice. This was done in order to determine the error involved in locating tongue boundaries. Areas were reported to one decimal place. In Edmonton, tongue areas for each slice of 5 subjects' tongue scan series, which were also measured in Winnipeg, were repeated. This was done in order to establish if there was any systematic error or bias between area measurements in Winnipeg versus Edmonton. Using a paired "t" test, statistical comparison was performed between 1) area measurements computed in Winnipeg, using its IVS, for each slice of the tongue scan series for these 5 subjects and 2) area measurements computed in Edmonton, using its IVS, for the same slices of the tongue scan series for these same 5 subjects and will be discussed 
under "Results" (page 89). In addition, as in Winnipeg, repetitions of area measurements were done in order to determine investigator error involved in the location of tongue boundaries. Since in Edmonton, areas were reported as rounded off whole numbers rather than to one decimal place, more repetitions were required to establish measurement error due to the loss of one significant figure. Therefore, for 12 subjects, two slices, selected at random, were measured twice, while for 10 subjects (including the above 5 subjects repeated in Winnipeg and Edmonton), two slices, selected at random, were measured four times.

In order to check that the IVS in Winnipeg did indeed report the true area as outlined, on a one to one basis, a phantom study was carried out. Six objects on the actual phantom, four circles consisting of different materials, the $E$ and of the word EMI, were measured using Mitutoyo* dial calipers (Figure 9). Diameters of the circles and the height and width of the back of the $E(I E, E)$ and the height and width of the ( $\mid \overrightarrow{\mid}, \overrightarrow{\mid}$ ) were measured five times each. The actual areas were calculated and the means and standard errors established. Five standard scans of the phantom taken at different times, were used to measure the areas of these same objects using the IVS ROI software program. Again, means and standard errors were established and are reported under "Results" (page 86). These objects were not measured on phantom scans using the IVS in Edmonton. It was felt that tongue areas measured on each slice of 5 subjects'

* Mitutoyo (Japan) No. $505-646$ 
Figure 9. Standard Phantom Scan.

Circles 1, 2, 3 and 4 and Water Bath (WB).

Figure 10. High Resolution Phantom Scan. 

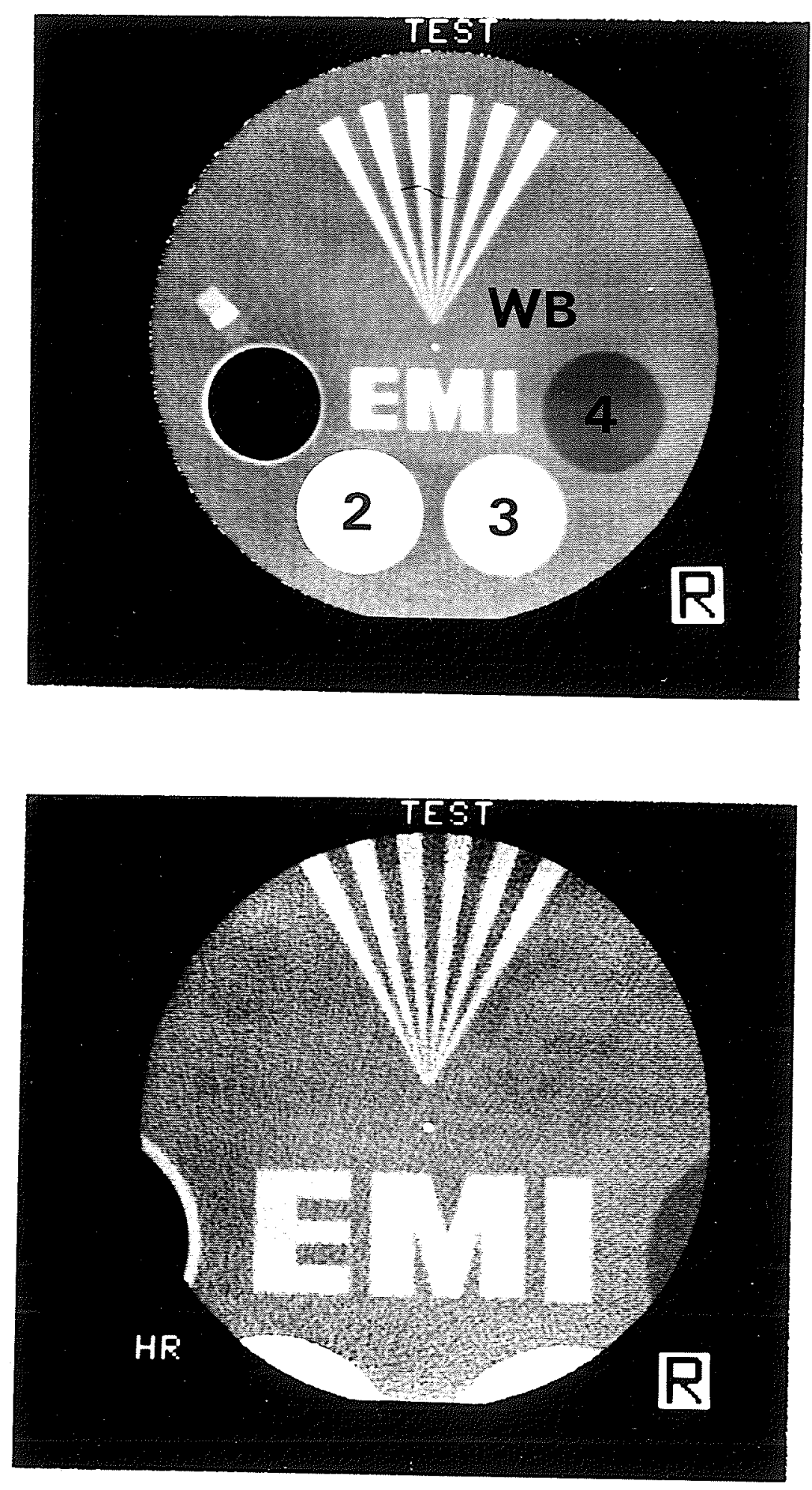
tongue scan series in both Winnipeg and Edmonton, as explained previously in this section, would establish adequately if the Edmonton IVS measured areas as the actual area.

The tongue scan series are all high resolution scans, which have a field size of 12 centimeters, as compared to the standard scan where the field size is 24 centimeters. Although the field size is decreased for high resolution scans, when viewed on the video screen of either the scanner or the IVS, they take up the same space as the standard scan and therefore the anatomical parts pictured are magnified. The IVS ROI software program in Winnipeg does not take into account that the tongue scan series are high resolution scans, rather than the more usual standard scans, and reports areas as if measuring a standard scan. Therefore, areas reported are larger than they should be. A phantom study was carried out to determine by what factor areas were magnified on high resolution scans when measured on the IVS in Winnipeg. Two high resolution phantom scans taken at different times were measured using the IVS. Only the $E$ and were measured, as described previously, because as the field size is decreased, parts of the circles are excluded (Figure 10). The $E$ and were measured five times each on the two high resolution phantom scans, these measured areas divided by the actual areas, as found previously, and a mean magnification factor and standard error established. The mean magnification factor was determined to be 4.0 with a standard error of \pm 0.05 . Therefore, all areas measured on high resolution scans using the IVS in Winnipeg were divided by 4.0 to account for magnification. 
This was not necessary for areas measured using the IVS in Edmonton, since the ROI software program compensated for magnification between a high resolution scan or a standard scan.

One difficulty encountered when measuring tongue areas is defining accurately the boundaries of the tongue. A knowledge of the anatomy of the area is essential and can be gained by studying various anatomical texts. An atlas of cross-sectional anatomy correlating images obtained by CT scanning to diagrams of human specimens proved very useful (Carter et al., 1977) when studying subject tongue scan series. In addition, dissection of cadaver tongues, to be discussed later, was very helpful in studying the anatomy and verifying what was actually seen on the tongue scan series of these cadavers. A typical tongue scan series for one subject and correlative anatomy are illustrated in Figure 12 .

Besides the above, the IVS Multiplanar Transformation (MPT)* software package was used to aid in tongue boundary identification. MPT software is designed to combine closely spaced series of transverse CT slices to present sagittal and coronal reconstructed planes. The slices necessary for each tongue scan series are loaded onto the disc from the magnetic tape in a special format and displayed sequentially by the MPTl loading program. Transformations are achieved by the MPT2 calculation program which outputs a transverse slice, the selected coordinate overlay, the selected sagittal section and the selected coronal section in $A, B, C$, and $D$ buffers respectively (Figure 11).

* MPT - Developed by T.Peters at the Montreal Neurological Institute in collaboration with EMI Medical Inc. 


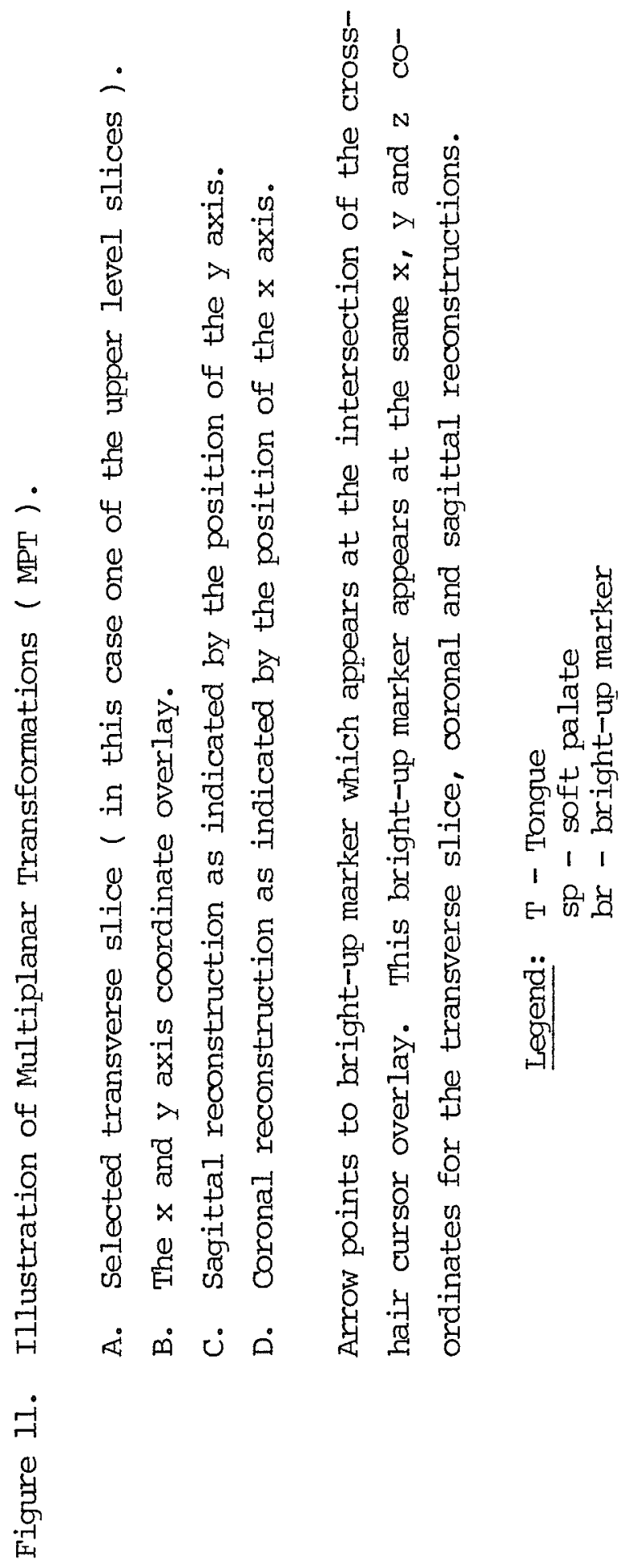



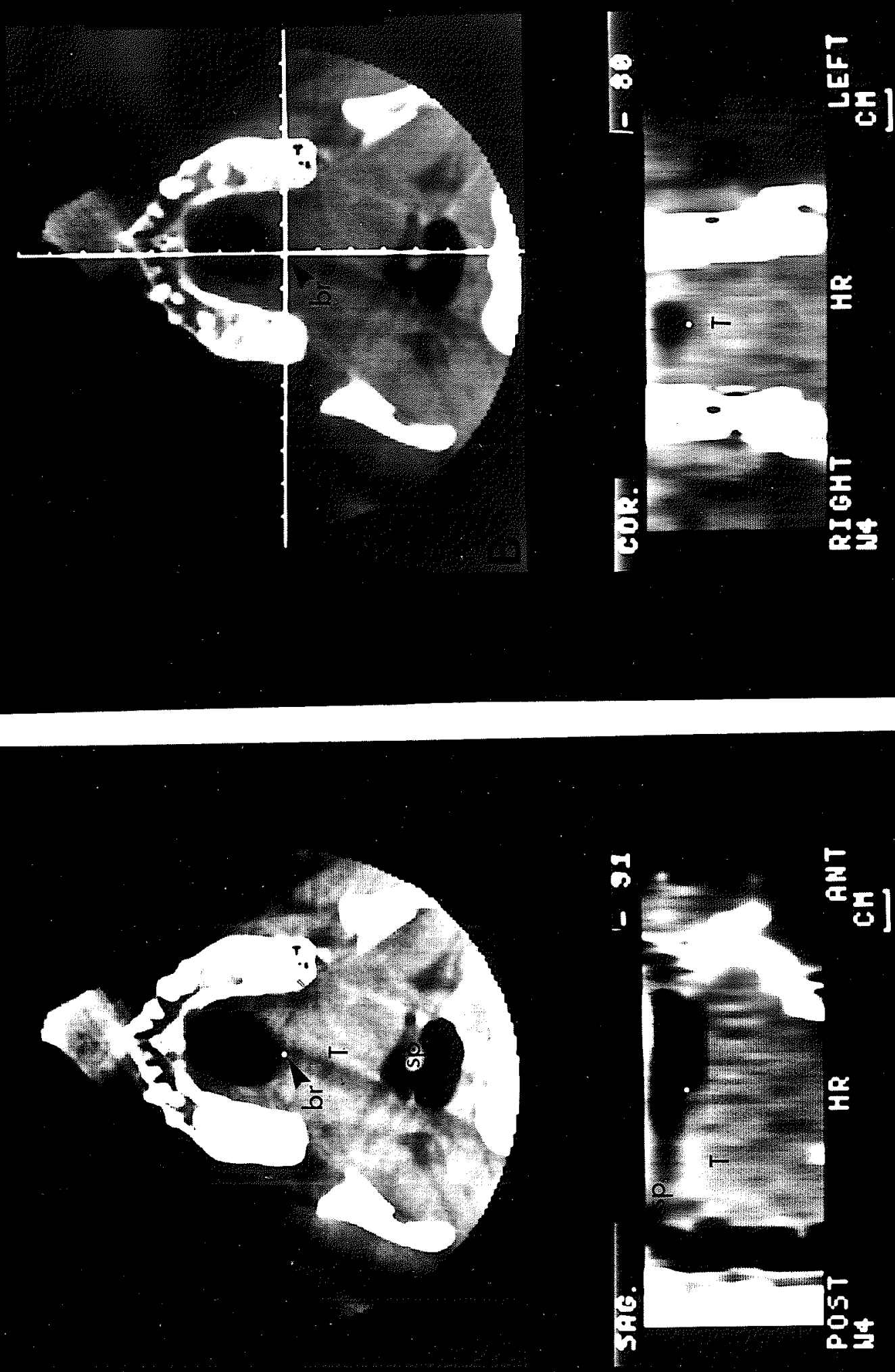
Buffer in this case refers to that part of the computer disc which holds the transverse slice, sagittal or coronal reconstruction. That is, buffer A will display the first uppermost transverse slice or any selected transverse slice on the screen monitor. Switching to buffer B produces cross-hair cursor lines ( $x$ and $y$ coordinate lines) over the image, which cross at the 80,80 midpoints of a $160 \times 160$ matrix array. Gradations on the cross-hair cursor are at 10 pixel intervals. Buffer C presents the sagittal reconstruction of all the slices of a subject's tongue scan series, as indicated by the position of the $y$ axis, as viewed from the left. Buffer $D$ presents the coronal reconstruction of all the slices of a subject's tongue scan series, as indicated by the position of the $x$ axis, as if viewed from the front of the subject. The $\mathrm{x}$ and $\mathrm{y}$ axes can be moved to any coordinate (1-160) and various sagittal and coronal planes displayed in the $C$ and $D$ buffers. A spatial marker is helpful in tongue boundary identification. While viewing transverse scans with the cross-hair cursor overlying the image, typing "P" on the keyboard beneath the video screen will cause a bright spot or bright-up marker to appear on the current transverse image, located at the intersection of the cross-hairs. This bright-up marker appears at the same $\mathrm{x}, \mathrm{y}$ and $\mathrm{z}$ coordinates when viewing sagittal or coronal reconstructions in their appropriate buffers (Figure 11 ). Therefore, using the spatial marker one can switch from transverse to sagittal or coronal modes and localize a particular boundary. Multiplanar reconstructions were obtained for each subject and once satisfactory tongue boundaries were established, ROI functions, that is, 
area measurements were performed.

The best multiplanar reconstructions can be obtained if contiguous slices of the patient are close together. High resolution scans require that 5 millimeter or smaller slice increments be used to extract the full benefit of the inherent resolution in the transformed pictures. Patient cooperation in not moving during scans is important, since there is no method currently of compensating for bad slices in the reconstruction of the series.

In addition to measuring tongue areas, the area of the space around the tongue (oral cavity, oropharynx) was also measured for every slice in which the tongue appeared. Identification of boundaries of the space area was less difficult than identifying tongue boundaries, since the space of the oral cavity or pharynx appears black and is more clearly delineated than the tongue itself. It was decided unnecessary to conduct a measurement error study when measuring space areas, since this was done adequately for tongue areas and measuring space areas was conducted exactly in the same manner as for tongue areas.

Once tongue areas and space areas were measured, the addition of the tongue pius space area gave the total oral area in which the tongue was contained in a particular slice.

G) Defining Anatomical Boundaries of the Tongue:

This can be explained most easily in conjunction with Figure 12, representing a typical tongue scan series for one subject. The over- 


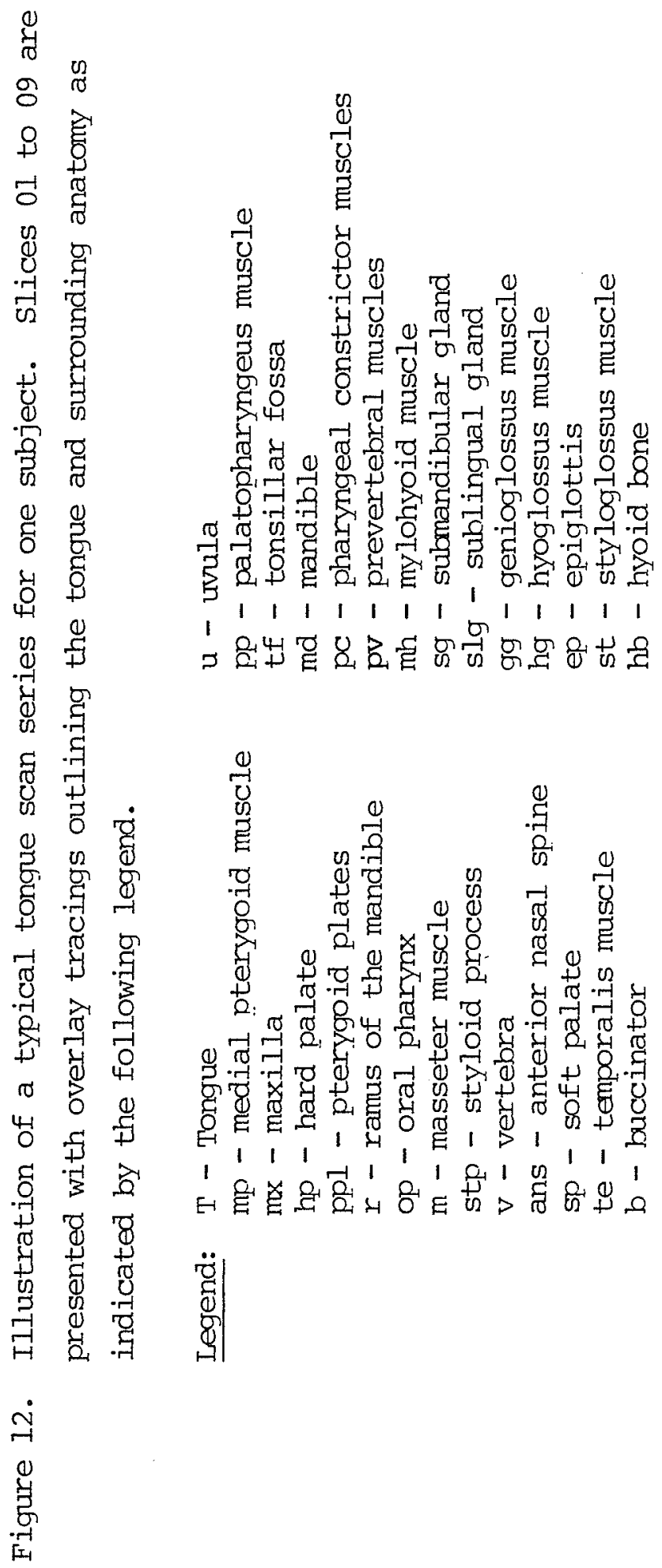



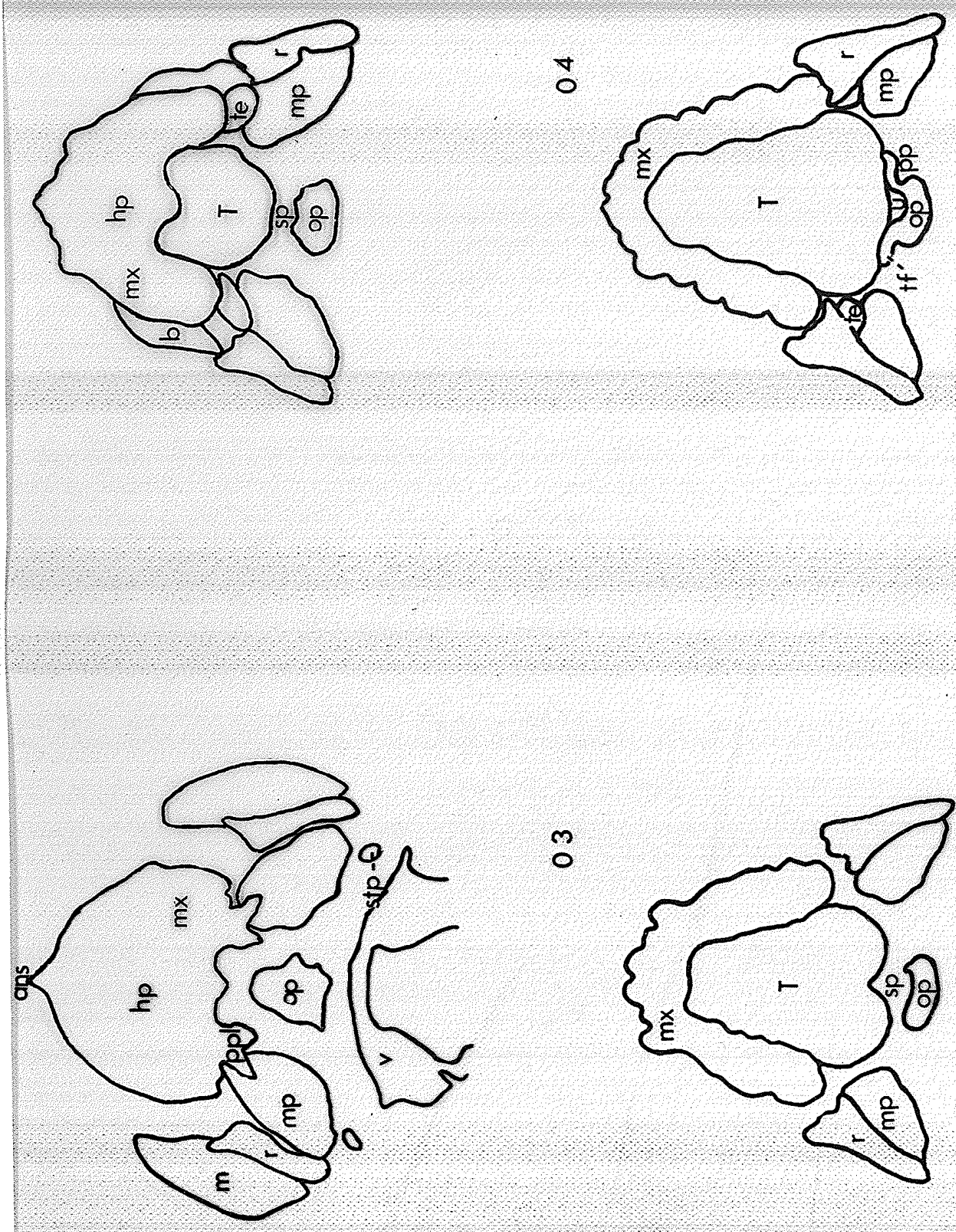

0

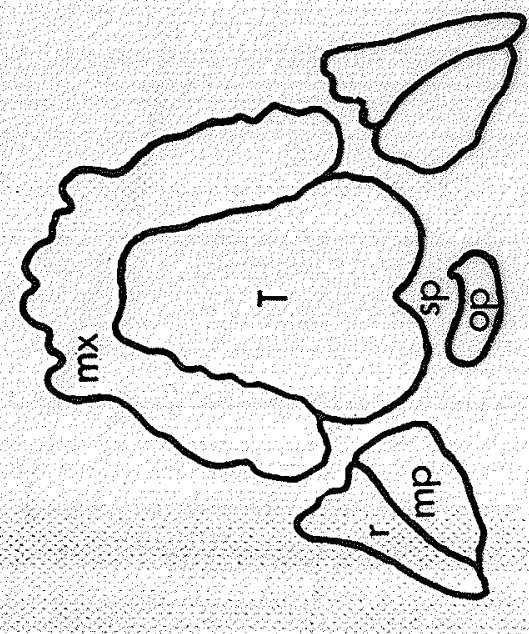


54
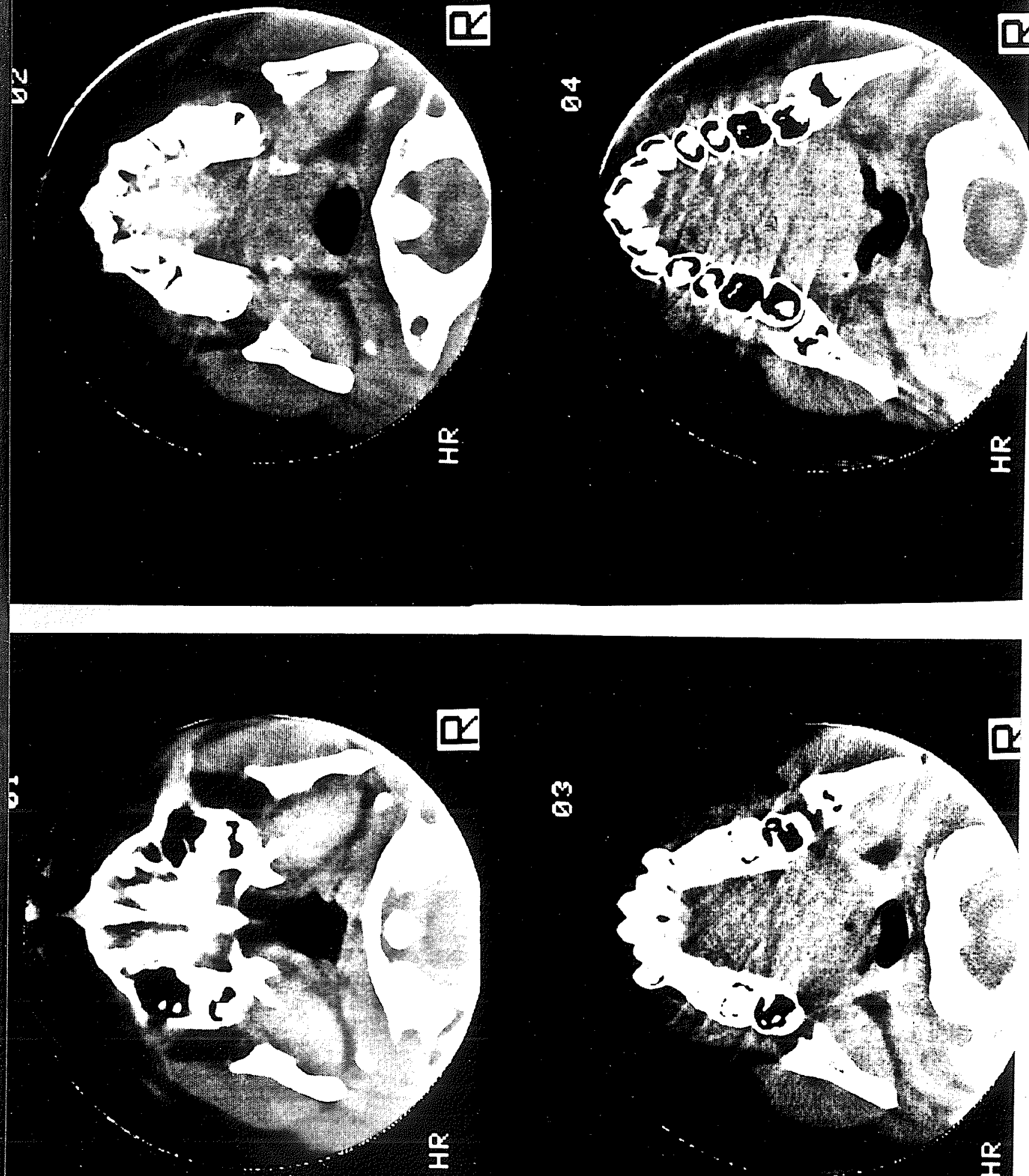

$m$

4

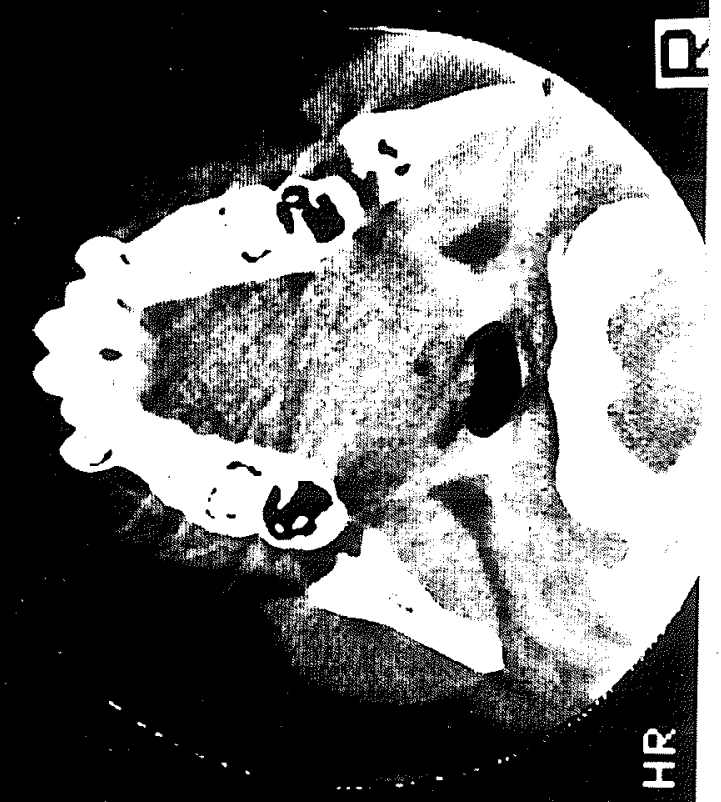


54

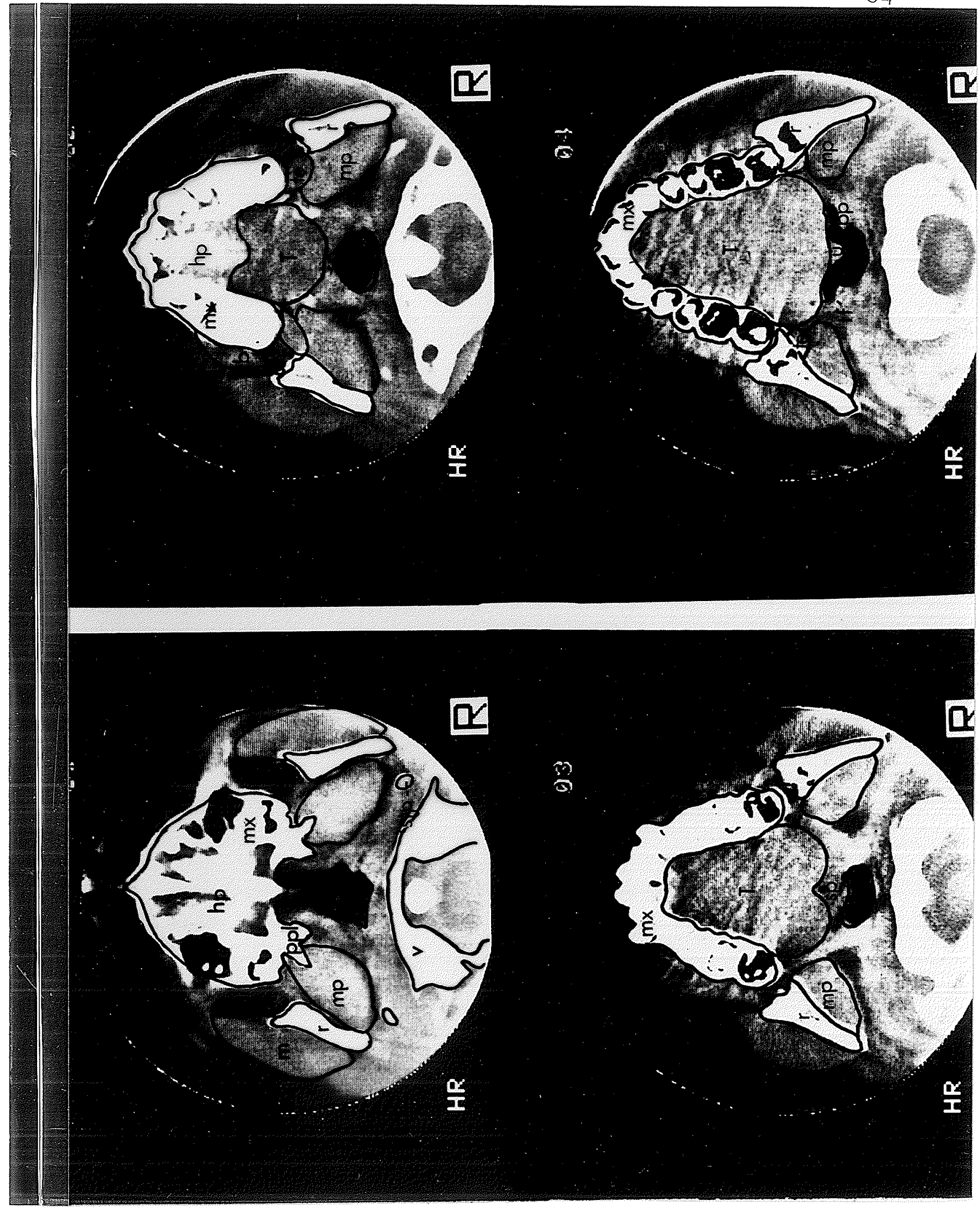



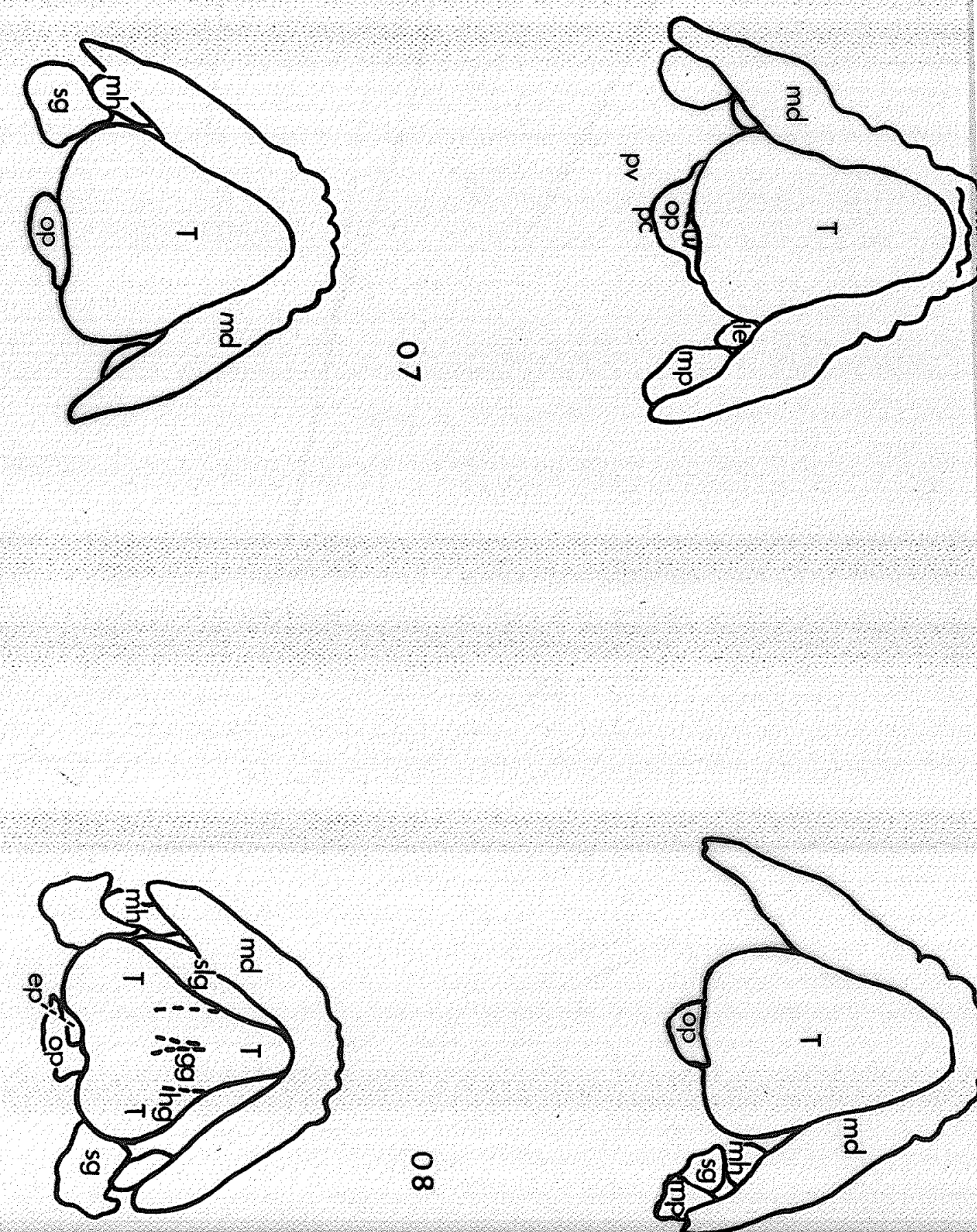

$\infty$

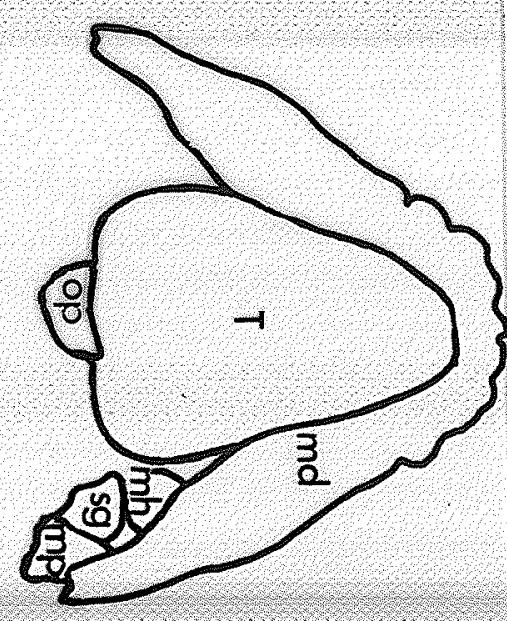



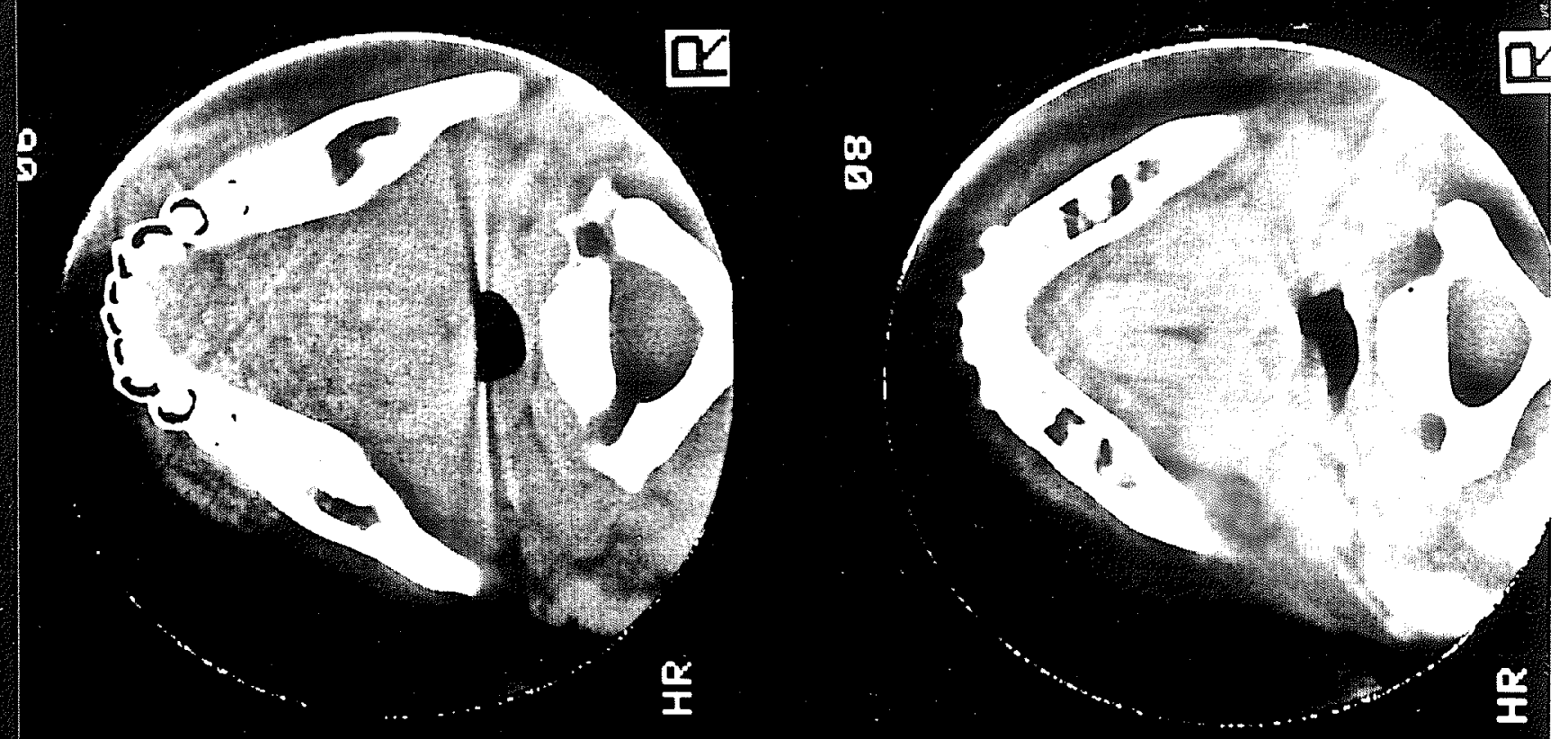

1

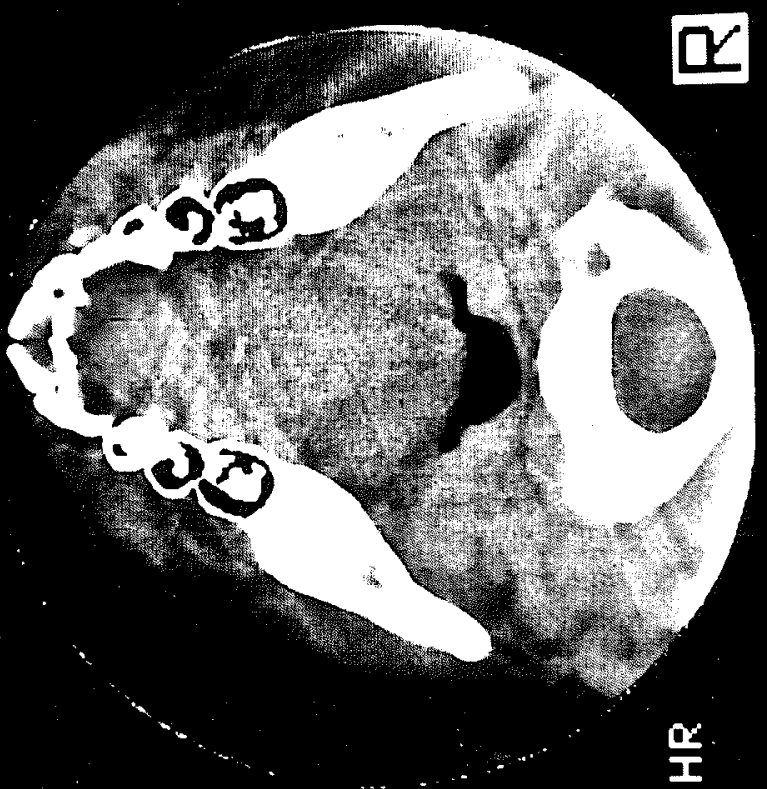

n

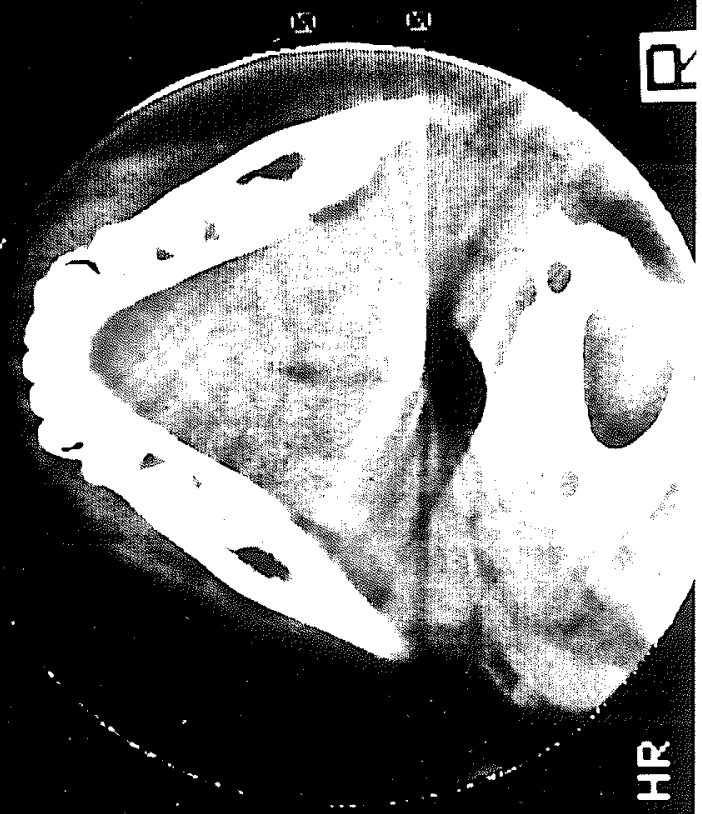


55

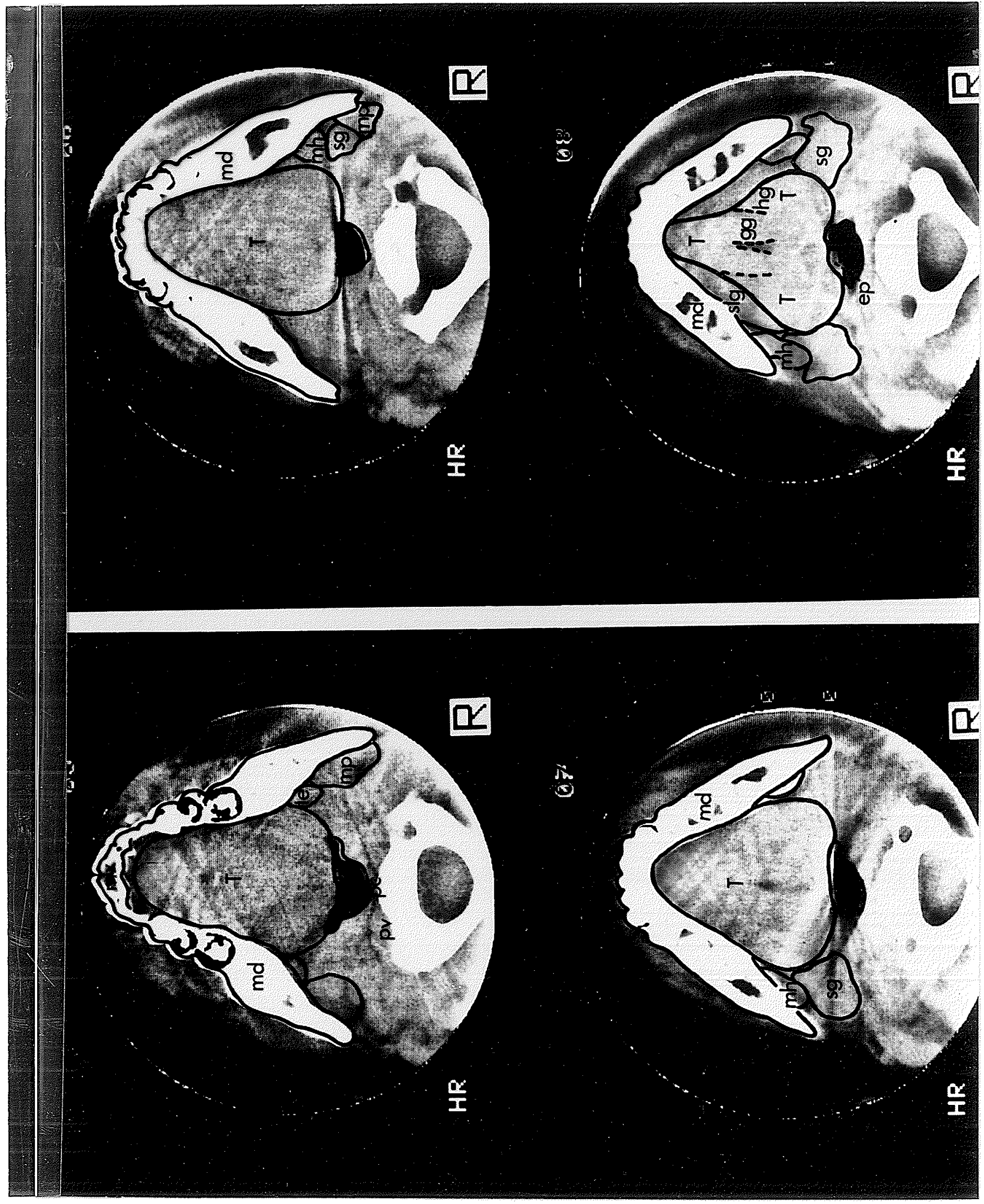



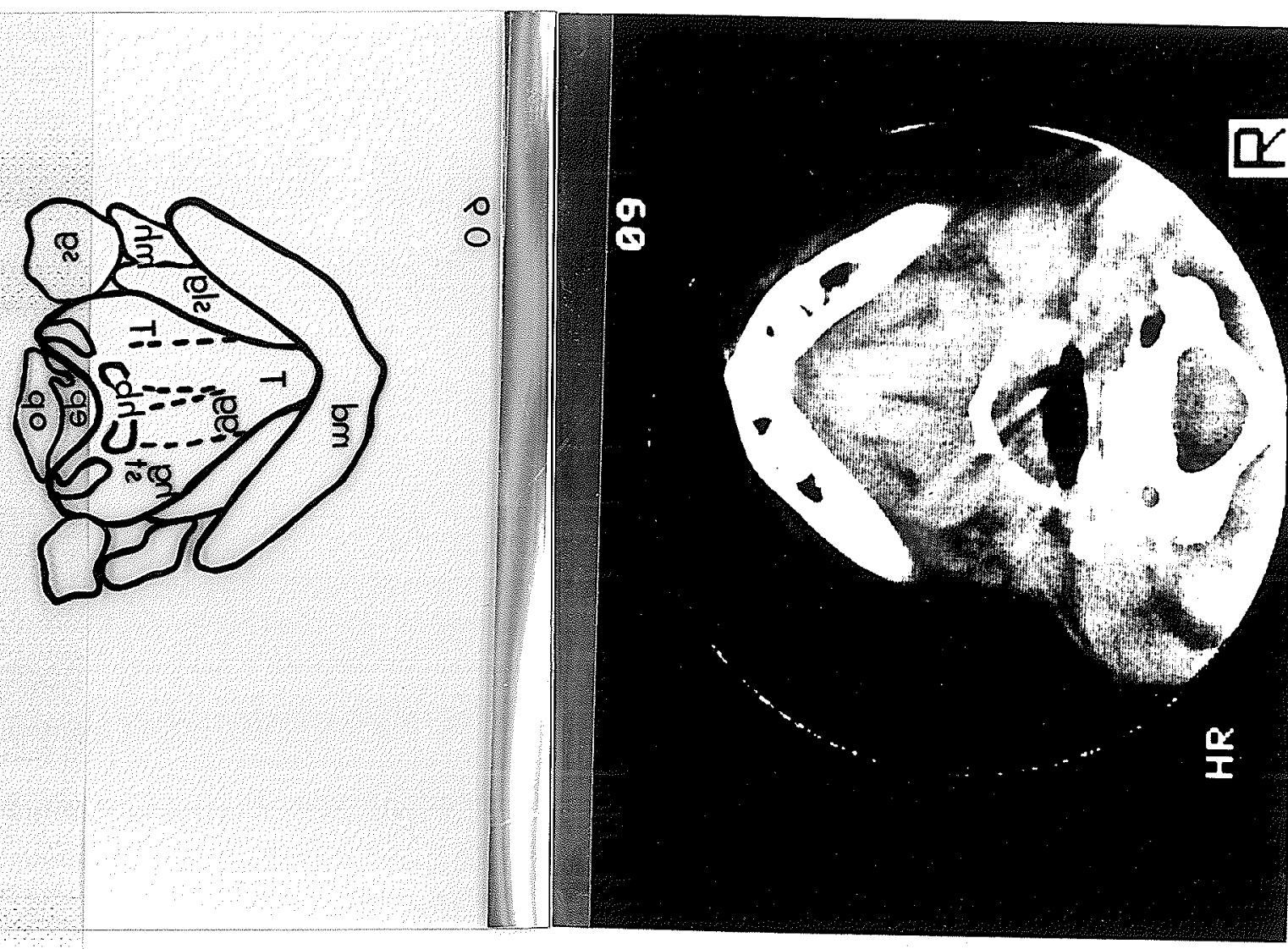


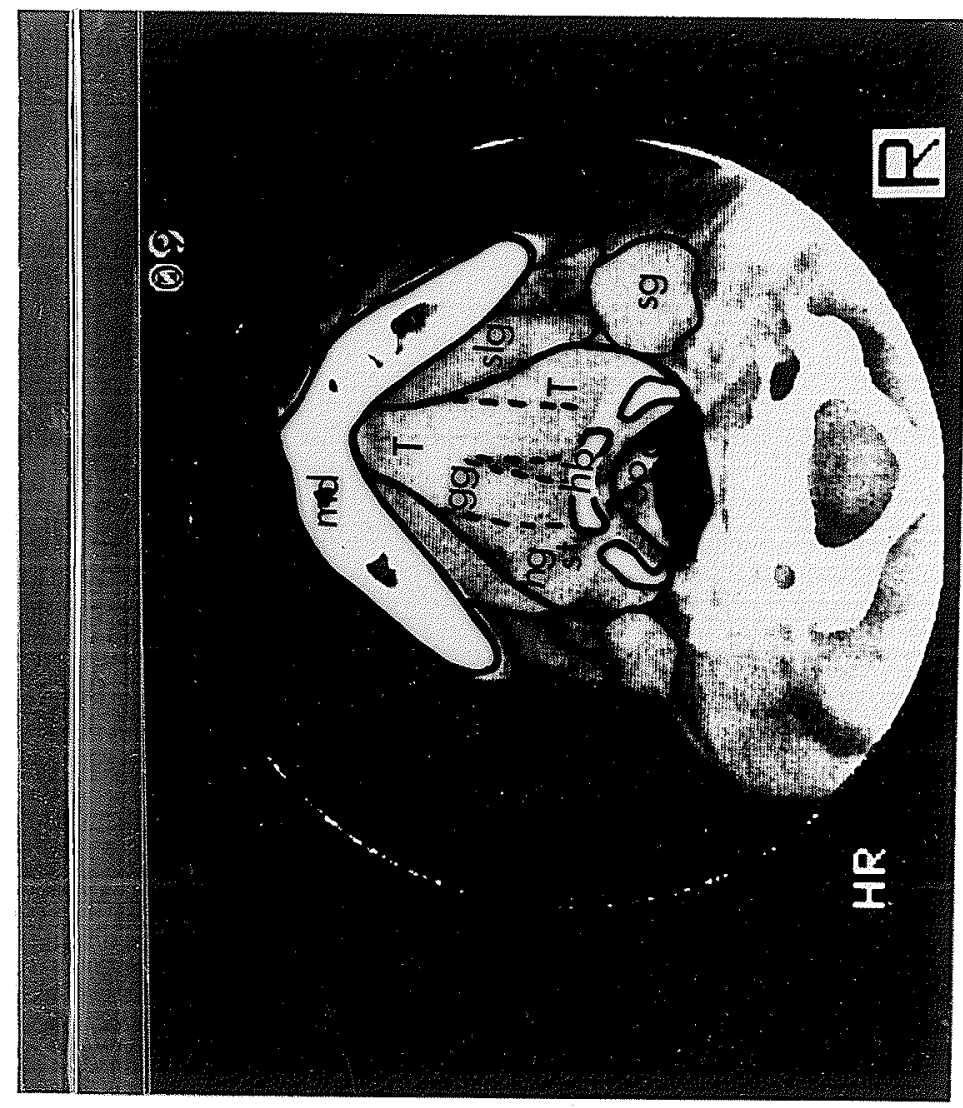


lay tracings were made using the original films of a tongue scan series. The photographs in Figure 12 are reproductions of the original $x$-ray film. For this reason, there is some loss of clarity of the photographs and there are some dimensional discrepancies between the photographs and overlay tracings. Landmarks will be described in terms of anterior, lateral, posterior and posterior lateral boundaries. Slice number 01 illustrates the maxilla, hard palate, pterygoid plates, ramus of the mandible, medial pterygoid and masseter muscles very clearly. The posterior border of the horizontal lamina of the palatine bone can also be seen and posterior to this the soft palate just begins to appear. There is no tongue apparent in this slice and this was confirmed by viewing sagittal and coronal reconstructions of the tongue scan series for this subject.

Slice number 02 shows much the same anatomy as the previous slice, except the pterygoid plates have almost disappeared and only the anterior one-half of the hard palate is visible. The fact that there is tongue in this cross-sectional slice was confirmed by using sagittal reconstructions and the bright-up marker as explained previously. The anterior and posterior outline of the tongue, defining where the hard palate began anteriorly and where the soft palate began posteriorly, was confirmed using sagittal reconstructions. Laterally the tongue boundary is the bony maxilla. A space, which appears darker relative to muscle, can be seen between the posterior lateral edge of the tongue and the medial pterygoid muscle and defines clearly this boundary. This darker appearing zone is due also to connective 
tissue and fat around the medial pterygoid muscle.

Slice number 03 contains a greater proportion of tongue than 02 . Anteriorly and laterally the boundary is the alveolar process of the maxilla while posteriorly it is the soft palate. A faint darker area can be seen along most of the boundary between the tongue and soft palate. This represents a slight air space between these structures, air appearing darker in a scan relative to soft tissue. Again, this posterior boundary was confirmed using sagittal reconstructions. The lateral posterior boundary of the tongue was placed in the darker zone between the muscles of the tongue and soft palate. In both previous slices, if the tongue had been positioned such that it was held away from the maxillary alveolus anteriorly or if it touched the hard palate only at the sides and not the center, areas where the tongue was not touching would be air spaces. These spaces would appear black with transition zones at the edges of the tongue.

Slice number 04 shows the alveolar process of the maxilla as the anterior and lateral boundary of the tongue. The tongue is not outlined following the indentations between the teeth, rather as a straighter line just skimming the alveolus. Posteriorly, the soft palate has almost disappeared with only the uvula present. Coronal and sagittal reconstruction confirm that this is uvula. Part of the posterior boundary next to the oropharynx is very clear. The lateral posterior boundary of the tongue can be seen in the darker zone separating the temporalis, medial ptyergoid and pharyngeal muscles from the tongue. 
The posterior and posterior lateral boundary of the tongue in slice number 05 is similar to 04 with only the tip of the uvula present. The mandible is now seen entirely, surrounding the rest of the tongue, with the maxillary teeth present anteriorly only.

In slice number 06, the uvula is no longer present. The posterior lateral boundary is more difficult to see in this particular slice than previous slices. Moving laterally from the oropharynx, the posterior boundary of the tongue usually sweeps straight out or slightly backward in a smooth line. Sometimes the separation of the tongue from pharyngeal muscles is clearer in other subjects than in this subject's slice. The posterior lateral boundary of the tongue occurs usually at the buttressed portion of the mandible formed by the oblique line on its external surface and the mylohyoid line on its internal surface. The medial pterygoid muscle can be seen and careful inspection reveals the temporalis muscle, where it attaches along the anterior border of the ramus of the mandible almost to the last molar tooth, or the mylohyoid muscle, depending on the plane of the slice. The darker zone between the temporalis or mylohyoid muscle and the tongue is an indicator of the posterior lateral boundary of the tongue. Therefore, from the posterior lateral edge to the posterior edge next to the oropharynx, the tongue can be outlined as a smooth curvature. This boundary was difficult to define even with the aid of sagittal and coronal reconstructions. The cadaver dissections were most helpful in verifying these boundaries on cadaver scans and subsequently relating them to subject scans. 
Slice number 07 shows the two bellies of the genioglossus muscle just beginning to appear as they extend into the main body of the tongue centrally. The posterior and posterior lateral boundary are bounded by the oropharynx, mylohyoid muscle, submandibular gland and pharyngeal muscles.

In slice number 08 the tongue appears to have a pear shape. The two bellies of the genioglossus muscle are clear with the hyoglossus and styloglossus muscles lateral to it, although these latter two muscles cannot be seen separately. The sublingual gland, mylohyoid muscle and submandibular gland can be seen laterally and posteriorly to the tongue. A portion of the epiglottis is also visible.

Slice number 09 illustrates the same features as 08 but more clearly. The tongue outline appears pear shaped and the hyoid bone and epiglottis are completely visible now. In some subjects, where the angle of the slice was lower anteriorly, the geniohyoid muscle appears at the same time as the hyoid bone. The geniohyoid muscle can be differentiated from the genioglossus muscle by its wider muscle bellies. Also, the hyoglossus and styloglossus muscles are not clear at this point and generally less mandible is present. In this particular subject, scans were taken at an angle such that when the hyoid bone was seen, the genioglossus muscle was still present. This was indicated by no change in size of the muscle bellies from the previous slice.

Generally, for all tongue scan series, a hint of the genioglossus was first seen in one slice at the lower end of the series and then 
more clearly visible for two or three slices beyond that. After these slices the geniohyoid muscle was usually seen. On the whole, tongue boundaries were most difficult to define for slices similar to 06 and 07 of this series and usually at the posterior and posterior lateral aspect. The lowermost boundary of the tongue was defined by the last slice in which the genioglossus muscle appeared.

H) Volume Calculation:

After area measurements were completed, each subject had a series of tongue areas and oral areas (area within which the tongue is contained for a particular slice). Knowing tongue areas and oral areas for a series of contiguous slices and knowing the thickness of each slice, it was possible to calculate the necessary volumes using the following mathematical formula:

$$
V=\frac{D}{2}\left[2 \sum_{2}^{n} A_{j}+A_{l}+A_{n}\right]
$$

where $\mathrm{V}=$ Volume

$$
A=\text { Area per slice }
$$

$D=$ Distance between each slice

$$
V=D \cdot \frac{A_{1}+2\left(A_{2}+A_{3}+\ldots A_{9}\right)+A_{10}}{2}
$$

For derivation of this formula, see Appendix III. Therefore, for each subject, a tongue volume and an oral volume 
(volume within which the tongue is contained) was calculated. A ratio

was calculated for each subject: $\frac{\text { tongue volume }}{\text { oral volume }} \times 100 \%$ to obtain a value representing the proportion of tongue volume relative to oral volume. Correlations of this ratio were made with model and cephalometric variables representing various craniofacial dimensions. These will be described later.

I) Cadaver Study:

Although precautions were taken to keep head position standardized from subject to subject for the tongue scan series, it was expected that variations would occur from subject to subject. It was not possible to conduct more than one tongue scan series for each subject, since this increases the amount of radiation exposure to the subject. Instead, three cadaver heads were obtained from the Anatomy Department of the University of Manitoba in order to conduct repeated tongue scan series on each cadaver head. Four tongue scan series were generated for one cadaver head and three tongue scan series for two cadaver heads. Between each series, the cadaver head was removed from the scanning unit and then replaced and repositioned in the head holder at the same orientation for the next series. This checked the effect that different head positions had on measurement of tongue volume. Subsequent area measurements and volume calculations, as described previously, were carried out for each tongue scan series for the three cadaver heads. A mean and standard error of tongue volume was obtained for each cadaver tongue and are reported under "Results" 
(page 90$).$

Each cadaver tongue was then carefully dissected, according to the anatomical boundaries outlined previously for tongue scans (Section G, page 53). Each tongue was dissected posteriorly down to the level of the epiglottis but excluding the epiglottis. The superior, anterior and lateral aspects were defined easily above the floor of the mouth. The inferior aspect was dissected and included the genioglossus, styloglosus and hyoglossus muscles. The geniohyoid muscle was excluded and the styloglossus muscle was severed at its exit from the main tongue mass. The mylohyoid muscle, sublingual and submandibular glands were also excluded. At its inferior aspect, the tongue took on the pear shape as described for the latter slices of a tongue scan series. The actual volume for each cadaver tongue was then obtained by the following method.

A 100 milliliter volumetric flask was weighed five times, in grams, using a Harvard Trip Balance* accurate to one decimal place. The volumetric flask plus 100 milliliters of distilled water was weighed five times and the weight per milliliter of water obtained. A 150 milliliter Pyrex beaker was weighed five times. An overflow can, especially designed to measure fluid displacement, was filled with distilled water to the point of overflowing from the spout. The cadaver tongue was then placed into the overflow can, while the 150 milliliter beaker caught the displaced water as it flowed out of the spout. Both beaker plus displaced water were then weighed. This was * Harvard Trip Balance, Florham Park, N. J., U. S. A. 
repeated five times for each cadaver tongue. The weight of the displaced water was calculated and by knowing the weight of a milliliter of water, a mean and standard error of the actual volume of each cadaver tongue was established. The above procedure is outlined by the following calculations:

$$
\begin{gathered}
\left(W t \cdot 100 \mathrm{ml} \cdot \text { Flask }+100 \mathrm{ml} \cdot \mathrm{H}_{2} \mathrm{O}\right)-(\text { Wt. } 100 \mathrm{ml} \cdot \text { Flask })=W t \cdot \text { per } 100 \mathrm{ml} \cdot \mathrm{H}_{2} \mathrm{O} \\
\text { Wt. per } 100 \mathrm{ml} \cdot \mathrm{H}_{2} \mathrm{O} / 100=\text { Wt. per ml. } \mathrm{H}_{2} \mathrm{O}
\end{gathered}
$$

(Wt.150ml. Beaker + Tong.Displ. $\left.\mathrm{H}_{2} \mathrm{O}\right)-($ Wt. 150ml. Beaker $)=$ Wt. Tong.

$$
\text { Displ. } \mathrm{H}_{2} \mathrm{O}
$$

(Wt.Tong. Displ. $\mathrm{H}_{2} \mathrm{O} \div$ Wt. per $\mathrm{ml} \cdot \mathrm{H}_{2} \mathrm{O}=$ Vol.Tong. (ml.)

These actual volumes were then compared to the volumes obtained by measurement of repeated CT scans of each cadaver tongue and are reported in the "Results" (page 90).

\section{J) Study Model Variables:}

A total of 12 measurements were made on study models for each subject, oriented and trimmed in centric occlusion (intercuspal position) with the aid of a wax bite. Linear measurements were made with Mitutoyo dial calipers to the nearest 0.01 millimeter and are listed as follows:

1. Overjet.

2. Overbite.

3. Open-bite.

4. Arch length - upper and lower. 
5. Intercanine width - upper and lower.

6. Intermolar width - upper and lower.

7. Arch circumference - upper and lower.

8. Palatal vault height.

A detailed description of these linear measurements can be found in Appendix IV. Each measurement was carried out twice for 32 subjects and in addition palatal vault height was measured four times for 17 subjects to test the accuracy of the instrument used to measure vault height.

An instrument for measuring palatal vault height was devised. One hole was machined into each end of a large Boley guage, through which pointed screws were placed in a fixed position. This holds a metal cross-bar from one leg to the other of the Boley guage. The metal cross-bar was slotted at each end, where it attached to each leg of the guage, to allow sliding from side to side as the legs were opened or closed (Figure 13). This assembly was positioned on the study models with the pointed screws in the central fossae of the permanent first maxillary molars and the legs of the guage supported parallel to the floor. The slotted metal cross-bar was positioned such that the dial calipers could be held vertically at the midpalatine raphe, in a seat especially prepared for it in the cross-bar (Figure 14). This height was recorded as total height. The height of the assembly itself was measured by placing it on a table top and measuring it in the same manner (Figure 15). The height of the assembly was then subtracted from the total height measurement obtained on the 


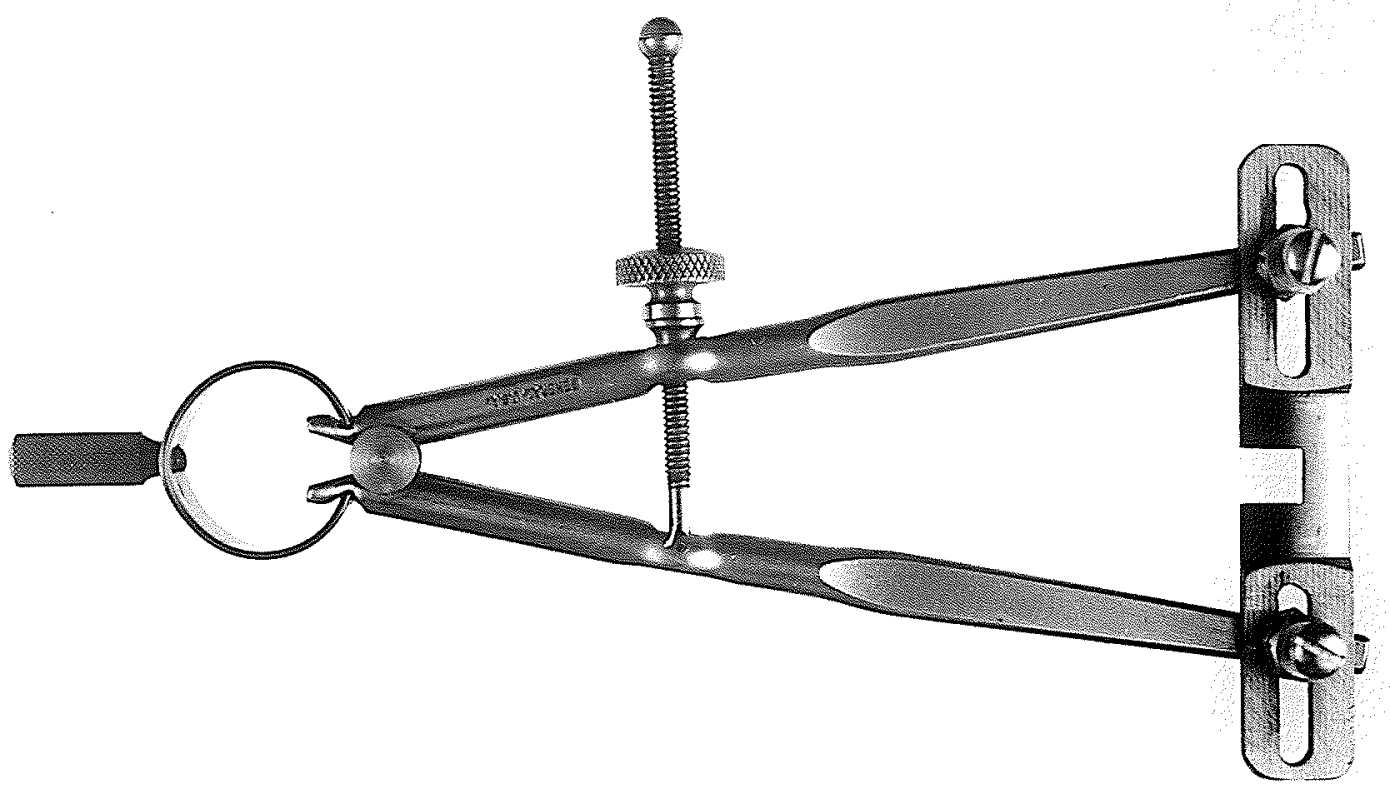

Figure 13. Illustration of the instrument devised to measure palatal vault height. 

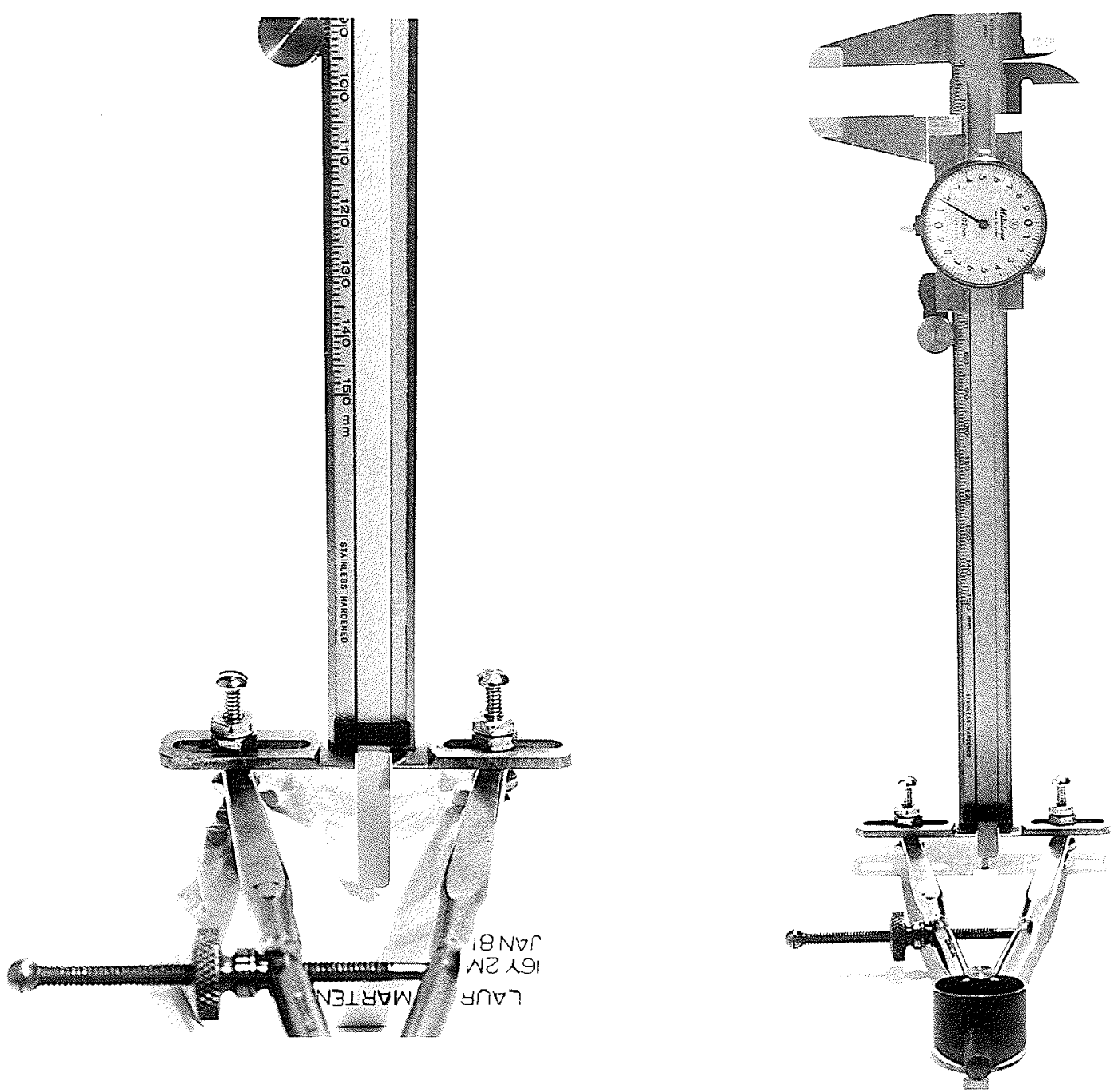

Figure 14. Illustration of positioning on the study

Figure 15. Illustration of measurement of the height of model of the instrument used to measure palatal the instrument itself on a table top, which vault height and dial calipers held verticalwas then subtracted from Iy at the midpalatine raphe in a seat especially prepared for it "total height" (as obtained in Figure 14) to obtain "palatal vault height".

in the cross-bar. Measurement obtained was recorded as "total height". 
study models in order to establish palatal vault height.

\section{K) Cephalometric Variables:}

Lateral cephalometric films were taken for each subject with the teeth in intercuspal position. The cephalometer consisted of a Picker* $x$-ray tube and Taylor* cephalostat, with a focal film distance of 152.4 centimeters (60 inches). Exposure were taken at 100 $\mathrm{mA}, 85 \mathrm{Kvp}$, for $7 / 10$ seconds. This cephalometer has been set up with exactly the same image geometry as the Moss cephalometrix*** cephalometer at the Faculty of Dentistry, University of Manitoba (Baker, 1981a), for which the magnification factor has been determined by Moir (1978) to be nine percent. Appropriate corrections for this magnification were performed during the process of analysis (Chebib et al., 1976).

Thirty three landmarks were used in this study as illustrated in Figure 16. Their description is found in Appendix V. The major planes used in this study are illustrated in Figure 17 and also described in Appendix V. All bilateral images of paired landmarks were midplaned and when a landmark could not be identified, it was omitted for the subject e.g. landmark 19 - tongue tip. A Ruscom**** logistics strip chart digitizer connected to a computer terminal was used to enter the "x" and " $y$ " coordinates for each cephalometric film

* Picker Canada Ltd., Serial No. N - 14056

** N. Taylor ( Engineering) Ltd., Parkstone - Dorset, England

$* * *$ Moss Corporation, Chicago, Illinois, U. S. A.

**** Ruscom Logics Ltd., Rexdale, Ontario, Canada 


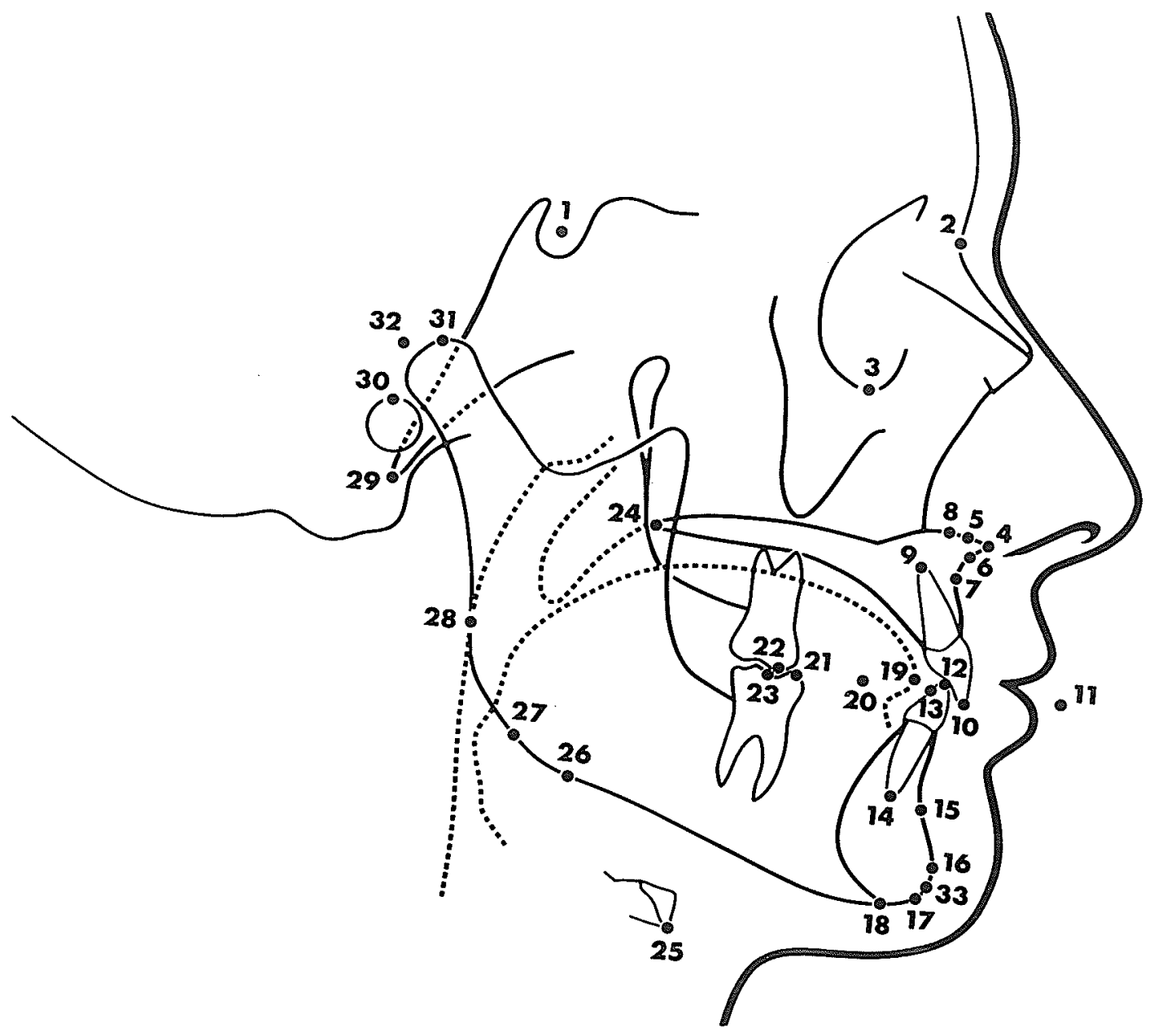

Figure 16. Illustration of the thirty three cephalometric landmarks used in this study. Their description is found in Appendix V. 


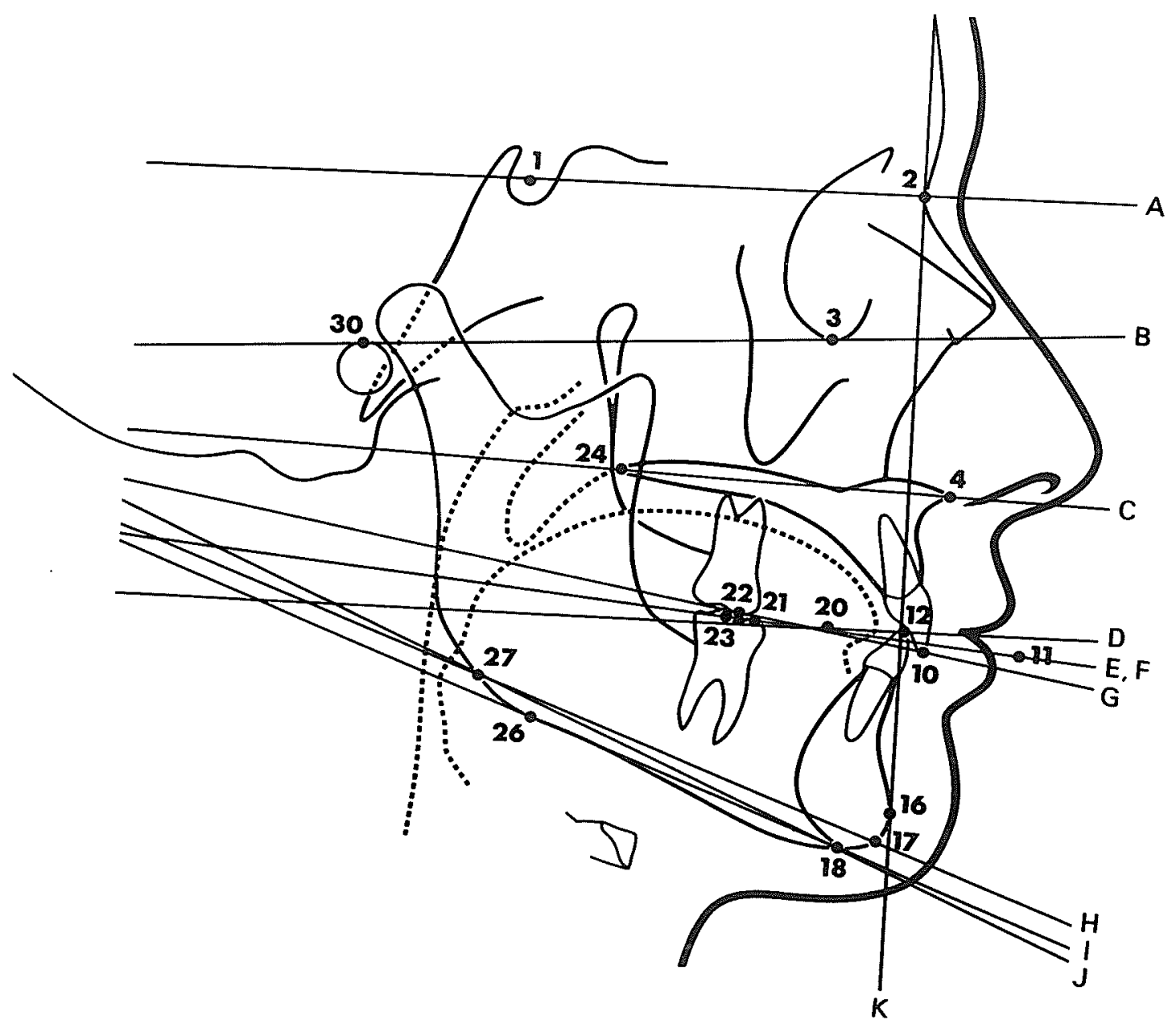

Figure 17. Illustration of the major cephalometric planes used in this study. Their description is found in Appendix V. 
landmark in a preselected sequence (Figure 16) into the University of Manitoba Computer (AmdahI/V7). The coordinates were transferred to the computer dise files using a specially written coordinate entry program via the TSO (time sharing option) system. Each cephalometric film for 32 subjects was digitized twice. Despite precautions, cephalometric films may have been taken at varying head extensions. Therefore, they were transformed to a standard orientation using the technique of Cleall and Chebib (1971). Based upon these standardized coordinates, linear and angular measurements used in this study were computed and stored for analysis in the University of Manitoba computer. All linear measurements were recorded in millimeters and all angular measurements in degrees.

Conventional cephalometric measurements were selected, in addition to measurements used in other studies dealing with the tongue and hyoid bone (Cleall, 1965), pharyngeal space (Vig and Cohen, 1974; Cohen and Vig, 1976), skeletal open-bite (Subtelny and Sakuda, 1964; Isaacson et al., 1971; Nahoum, 1971, 1975; Nahoum et al., 1972; Lowe, 1980), maxillary and mandibular length (Harvold, 1968; Harvold and Vargervik, 1971) and functional occlusal plane (Vasiga, 1972). These measurements are listed in Table 3 and described further in Appendix VI. 
Table 3. List of Linear and Angular Cephalometric Measurements. (Their description is found in Appendix VI) .

I Intermaxillary Ht., Lth. and Area, Tongue and Hyoid

Ant. I. M. Ht.

Post. I. M. Ht.

I. M. Lth.

I. M. Area

Vert. pos. of hyoid

Hor. pos. of tongue

II Cranial Base

SNBa

SN (mm.)

$\mathrm{SBa}$ (mm.)

III Maxilla to Cranium

$\mathrm{SN}-\mathrm{FH}$

SNA

$\mathrm{NA}-\mathrm{FH}$

Angle of Convexity (NAPg)

$\mathrm{SN}-\mathrm{OP}$

SN-UOP

SN-FOP

$\mathrm{SN}-\mathrm{PP}$

ANS-PNS (mm.)

MxUL (mm.)

IV Mandible to Cranium

Facial Angle

$\mathrm{SNB}$

SN-IOP

$Y$ axis

SN-GoGn

SN-GOMe

SN-IBMe

Gonial Angle (sup. condyle-GoGn)

Corpus Ith. (GoGn, mm.)

MaUI (m.) 


$$
\begin{aligned}
& \text { ANB } \\
& \text { OP-GOGn } \\
& \text { OP-GOMe } \\
& \text { OP-LBMe } \\
& \text { UOP-GOGn } \\
& \text { UOP-GOMe } \\
& \text { UOP-IBMe } \\
& \text { LOP-GOGn } \\
& \text { LOP-GOMe } \\
& \text { LOP-LBMe } \\
& \text { FOP-GOGn } \\
& \text { FOP-GOMe } \\
& \text { FOP-IBMe } \\
& \text { PP-GOGn } \\
& \text { PP-GOMe } \\
& \text { PP-LBMe } \\
& \text { PP-OP } \\
& \text { PP-UOP } \\
& \text { PP-IOP } \\
& \text { PP-FOP } \\
& \text { ULD (mm.) }
\end{aligned}
$$

VI Dentoalveolar
U. I-SN
U. 1-NA
U.I to SN (mm.)
U.I to PP (mm.)
U.I to NA (mm.)
$\mathrm{U} .1$ to $\mathrm{APg}$ (mm.)
ADH (mm.)
U. 6 to SN (mm.)
U. 6 to PP (mm.)
$\mathrm{U} .6$ to $\mathrm{PP} \perp \mathrm{OP}$ (mm.)
U. 6 to PP $\perp$ FOP (mm.)
U. 6 to PP $\perp$ UOP (nm.)
L. 1-GoGn
L. 1-GOMe
L. I-IBMe
L. 1-NB
L. 1 to GoGn (nm.)
L. 1 to GoMe (mm.)
I. 1 to IBMe (m.)
L. 1 to NB (m.)
L. 1 to APg (mm.)
L. 6 to GoGn (mm.)
L. 6 to GoMe (mm.)
I. 6 to IBMe (mm.)
U.I to L.I 


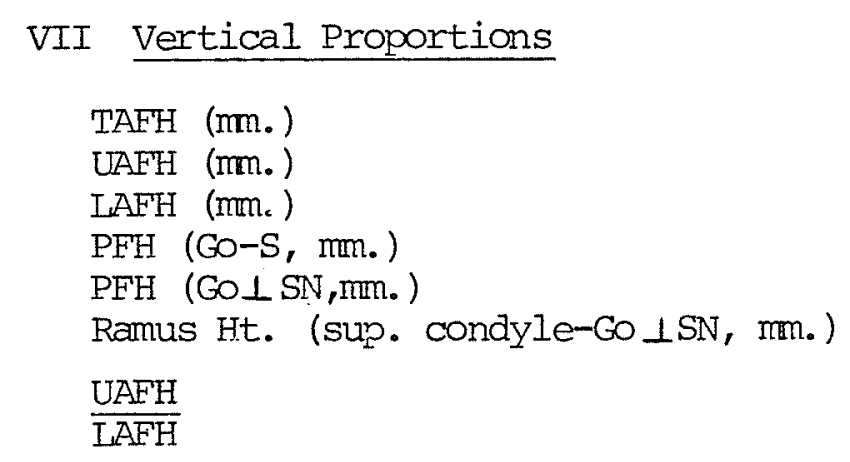




\section{L) Measurement of Error:}

1. Instrument Error:

Since development of a technique with which to measure tongue volume relative to the space surrounding it was the main intent of this study, the reliability of the instruments (CT5005, IVS) used to accomplish this task was important to establish. The error studies conducted to investigate this have already been outlined in "Materials and Methods" (sections E, F, I) and the findings will be discussed under "Results" (pages 84 to 92 ).

\section{Investigator Error:}

As part of the main investigation, three pilot studies were undertaken to assess the measurement error involved in 1) boundary identification of the tongue and the areas measured according to these boundaries using the IVS in Winnipeg and Edmonton 2) the location and measurement of study model variables and 3) linear and angular measurements obtained as a result of digitization of hard and soft tissue cephalometric landmarks.

i) Pilot Study 1: The first pilot study examined the measurement error on the part of this investigator in identifying tongue boundaries and measuring tongue areas when using the IVS tracker ball system to delineate the tongue. For 15 subjects measured in Winnipeg, two slices, selected at random, of each tongue scan series, were measured 
twice for a total of thirty slices. For 22 subjects measured in Edmonton (5 of which were also measured in Winnipeg), two slices, selected at random, of each tongue scan series, were measured twice for 12 subjects and two slices were measured four times for 10 subjects. It was decided to do more repetitions for some subjects, since the IVS in Edmonton rounded areas off to whole numbers rather than reporting areas to one decimal place as in Winnipeg. The estimation of measurement error was calculated as suggested by Chebib and Burdick (1973). The standard deviation of the error, $s$, was calculatd by:

$$
s=\sqrt{\frac{\sum_{i=1}^{n} \sum_{j=1}^{m}\left(x_{i j}-\bar{x}_{i}\right)^{2}}{n(m-1)}}
$$

where $n=$ total number of slices

$$
\begin{aligned}
& m=\text { number of repeated measures } \\
& \mathrm{X}_{i j}=\text { actual measurements } \\
& \bar{x}_{i}=\text { mean of } m \text { measurements of the ith slice. }
\end{aligned}
$$

This value was derived by subjecting the data to a one-way analysis of variance where the grouping is by $\mathrm{n}$ slices rather than by measurements. The $s$ is the square root of the error (within) mean square. The maximum error $\left(e_{p}\right)$ committed in 95 percent and 99 percent of the measurements is:

$$
e_{p}= \pm t_{(p, d f)} s
$$


where $k$ = number of readings on each slice of the final data

$$
\begin{aligned}
t= & \text { theoretical Student } t \text { value for df degrees of freedom and } \\
& \text { probability } p \\
d f= & \text { number of degrees of freedom associated with } s,[d f=n(m-1)] \\
= & 57
\end{aligned}
$$

The standard deviation of the error, 95 percent maximum error and 99 percent maximum error are shown in Table 4. The maximum error committed 99 percent of the time during boundary identification and area measurement was 0.63 square centimeters in Winnipeg and 1.98 square centimeters in Edmonton.

The systematic error, during area measurement, of the IVS in Winnipeg versus Edmonton will be discussed under "Results" (page 89 ).

For subsequent volumes obtained from area measurements of contiguous slices of tongue scan series for each subject, the error associated with each volume is calculated by:

$$
\frac{e_{p}}{\sqrt{n}}
$$

where $e_{p}=$ maximum error associated with probability $p$ for each slice $\mathrm{n}=$ number of slices used to calculate volume

The average number of slices measured for each subject was 9 slices with a range of 7 to 12 slices. Those volumes, derived from area measurement of 9 slices would be associated with a maximum error, 99 percent of the time, of 0.21 cubic centimeters for subjects measured in Winnipeg and 0.66 cubic centimeters for subjects measured in Edmon- 
Table 4. Standard deviation of the error, $95 \%$ maximum error and $99 \%$ maximum error committed during tongue boundary identification and area measurement on the Independent Viewing System (IVS) in Winnipeg and Edmonton.

\begin{tabular}{lccc}
\hline Location & $\begin{array}{c}\text { Standard Deviation } \\
\text { of Error }(\mathrm{s})\end{array}$ & $\begin{array}{c}95 \% \text { Maximu } \\
\left.\text { Error }( \pm \mathrm{cm})^{2}\right)\end{array}$ & $\begin{array}{c}99 \% \text { Maximm } \\
\left.\text { Error }( \pm \mathrm{cm})^{2}\right)\end{array}$ \\
\hline Winnipeg & 0.2302 & 0.47 & 0.63 \\
Edmonton & 0.7459 & 1.49 & 1.98 \\
\hline
\end{tabular}


ton. The error may be calculated in a similar manner for those subjects where volumes were calculated from a number of slices $(n)$ other than 9 and are listed in Table 5.

ii) Pilot Study 2: The second pilot study examined the measurement error associated with study model variables. Each of the 12 variables studied was measured twice for each of the 32 study models. In addition, the variable entitled "palatal vault height" was measured four times for 17 of the 32 study models, in order to establish the error associated with the instrument devised to measure vault height. The estimation of measurement error was calculated as suggested by Chebib and Burdick (1973) according to the formulas outlined for Pilot Study 1. The standard deviation of the error, 95 percent maximum error and 99 percent maximum error are reported in Table 6 for all the model variables used in this study. The maximum error committed in 99 percent of the measurements was 0.93 millimeters.

iii) Pilot Study 3: The third pilot study examined the error associated with linear and angular measurements as a result of digitization of hard and soft tissue cephalometric landmarks. All cephalometric points were digitized twice for 32 subjects and the estimation of measurement error calculated as suggested by Chebib and Burdick (1973) according to the formulas outlined for Pilot Study 1. Ten angular measurements and five linear measurements were selected for this purpose and the standard deviation of the error, 95 percent maximum error and 99 percent maximum error reported in Table 7. The largest maximum error committed for angular measurements was 1.38 degrees for the 
Table 5. $99:$ maximum error for volumes calculated from contiguous slices where $\mathrm{n}=$ number of slices.

\begin{tabular}{ccc}
\hline Number of Slices $(\mathrm{n})$ & $\begin{array}{c}99 \% \text { Maximum Error }\left( \pm \mathrm{tm} .^{3}\right) \\
\text { Winnipeg }\end{array}$ \\
\hline & & \\
7 & 0.2381 & 0.7484 \\
8 & 0.2227 & 0.7000 \\
9 & 0.2100 & 0.6600 \\
10 & 0.1992 & 0.6261 \\
11 & 0.1900 & 0.5970 \\
12 & 0.1819 & 0.5716 \\
\hline
\end{tabular}


Table 6. Standard deviation of the error, $95 \%$ maximum error and $99 \%$ maximum error associated with study model variables.

\begin{tabular}{lccc}
\hline \multicolumn{1}{c}{ Variable } & $\begin{array}{c}\text { Standard Deviation } \\
\text { of Error (s) }\end{array}$ & $\begin{array}{c}95 \% \text { Maximum } \\
\text { Error (tmm.) }\end{array}$ & $\begin{array}{c}99 \% \text { Maximum } \\
\text { Error (tmm.) }\end{array}$ \\
\hline Overbite & 0.1783 & & 0.26 \\
Open-bite & 0.1426 & 0.26 & 0.41 \\
Overjet & 0.2414 & 0.35 & 0.47 \\
Arch Lth. - U & 0.2205 & 0.32 & 0.43 \\
Arch Lth. - L & 0.3241 & 0.47 & 0.63 \\
Intercanine Wth. - U & 0.1487 & 0.22 & 0.29 \\
Intercanine Wth. - L & 0.1395 & 0.20 & 0.27 \\
Intermolar Wth. - U & 0.3056 & 0.44 & 0.60 \\
Intermolar Wth. - L & 0.1917 & 0.28 & 0.37 \\
Arch Circum. - U & 0.4468 & 0.65 & 0.87 \\
Arch Circum. - L & 0.4793 & 0.69 & 0.93 \\
Palatal Vault Ht. & 0.1171 & 0.16 & 0.22 \\
\hline
\end{tabular}


Table 7. Standard deviation of the error, $95 \%$ maximm error and $99 \%$ maximum error associated with selected angular and linear cephalometric variables.

\begin{tabular}{|c|c|c|c|}
\hline Variable & $\begin{array}{l}\text { Standard Deviation } \\
\text { of Error (s) }\end{array}$ & $\begin{array}{l}95 \% \text { Maximum } \\
\text { Error (tmm.) }\end{array}$ & $\begin{array}{l}99 \text { \% Maximum } \\
\text { Error (tmm.) }\end{array}$ \\
\hline SNA (deg.) & 0.2980 & 0.43 & 0.58 \\
\hline SNBa (deg.) & 0.4050 & 0.59 & 0.79 \\
\hline $\mathrm{SN}-\mathrm{OP} \quad(\mathrm{deg} \cdot)$ & 0.2049 & 0.30 & 0.40 \\
\hline PP-FOP (deg.) & 0.3760 & 0.54 & 0.73 \\
\hline ANB (deg.) & 0.2933 & 0.42 & 0.57 \\
\hline U. 1 to $\mathrm{SN}$ (deg.) & 0.5666 & 0.82 & 1.11 \\
\hline U. 1 to $\mathrm{NA}\left(\mathrm{deg}_{.}\right)$ & 0.5109 & 0.74 & 1.00 \\
\hline U. 1 to L. 1 (deg.) & 0.7092 & 1.03 & 1.38 \\
\hline FOP-GoGn (deg.) & 0.3987 & 0.58 & 0.78 \\
\hline L. I to LBMe (deg.) & 0.4483 & 0.65 & 0.87 \\
\hline ANS-PNS (mm.) & $0.03] .6$ & 0.05 & 0.06 \\
\hline $\mathrm{SN} \quad(\mathrm{nm})$. & 0.0 & 0.0 & 0.0 \\
\hline MxUL (mm.) & 0.0316 & 0.05 & 0.06 \\
\hline TAFH (nTn.) & 0.0 & 0.0 & 0.0 \\
\hline U. 1 to NA (mm.) & 0.0316 & 0.05 & 0.06 \\
\hline
\end{tabular}


variable U.I to L.I. The largest maximum error committed for linear measurements was 0.06 millimeters. The error associated with linear measurements was small.

M) Statistical Analysis:

The sample consisting of 32 subjects was divided into two groups:

Group 1 - Overbite $\geq 0$ (referred to as nonopen-bite or N.O.B.)

Group 2 - Overbite $<0$ (referred to as open-bite or O.B.)

Group 1 contained 27 subjects and Group 2 contained 5 subjects. The significance between the means for each of the ratio $\frac{\text { tongue volume }}{\text { oral volume , }}$ cephalometric variables and model variables were determined using a Student's "t" test for the two groups and are reported in the "Results" (pages 93 to 97 ).

In addition correlation coefficients between $\frac{\text { tongue volume }}{\text { oral volume }}$ and each of the cephalometric and model variables were calculated from all subject data and are discussed in the "Results" (page98). 
Results

\section{A) Instrument Error:}

In order to check the reliability of the EMI CT5005 Scanner and the Independent Viewing System, which were used to obtain the tongue scan series and accomplish area measurements prior to volume calculation, a series of error studies were conducted. These are outlined in "Materials and Methods" (sections E, F, I) and the results are as follows:

\section{CT numbers:}

As mentioned in "Materials and Methods", the EMI CT5005 Scanner is subject to day to day variation or drift, which may compromise the use of CT numbers. As part of a quality control program, the CT scanner used in this study is checked to ensure that the mean $C T$ value for a phantom scan of a water bath is consistent from day to day. A phantom scan is generated each morning and the mean and standard deviation of the water bath checked and logged. Using quality control values obtained for the days of (if available) and for two days before and after subject and cadaver scans, the mean CT number for water was found to be -0.1143 with a standard deviation of \pm 1.1411 CT numbers. In EMI units (magnifying constant $=500$ ), a change of one CT number

corresponds to a 0.2 percent change in the linear attenuation coefficient (u) (McCullough, 1977). It is generally claimed that CT systems 
are capable of resolving tissues that differ as little as 0.5 percent in $\mu$ or 2.5 in CT numbers. While 0.5 percent is an optimal value, most devices operate under conditions that yield a precision closer to 1.0 percent (5 CT numbers) (Ter-Pogossian, 1977). Therefore, the CT number drift of \pm 1.1411 will not be detectable as a density change in the image recorded by the CT scanner.

It was desirable to check that the $\mathrm{CT}$ value for a water bath was not different when using the IVS than that computed and stored by the EMI CT5005. Five phantom scans, taken at different times, were selected and the region of interest (ROI) capability of the IVS utilized to obtain the mean CT number for a water bath for each phantom scan. The mean value for water for the five phantom scans was -0.6480 with a standard deviation of \pm 0.3604 . Both this mean and standard deviation are within the standard deviation obtained for the CT number of water resulting from quality control measurements. In other words, the CT value for water was not different on the IVS, as that originally computed and stored by the scanner.

A check on the sensitivity of the CT system (i.e. CT numbers) in detecting differences in inherent contrast (chemical composition) was carried out using phantom scans and is described in Appendix II.

To check that the IVS in Edmonton reported CT numbers that were not different from those reported on the IVS in Winnipeg, the CT numbers of approximately the same area of muscle of the same five slices of the same 5 subjects were compared. As discussed in "Materials and Methods," the CT number of muscle will vary from slice to slice and 
from subject to subject. The variability of CT numbers of muscle according to 1) variation among subjects 2) variation among slices within subjects and 3) variation according to location (Winnipeg versus Edmonton), when subjected to a mixed analysis of variance, revealed the following results (see Table 8 ). The mean square reported for "among subjects" shows that the CT number for muscle does vary considerably from subject to subject. For "slices within subjects" there is also great variability but less than for "among subjects". The variability in CT number "between locations" is not significant. Differences of greater than $5 \mathrm{CT}$ numbers "between locations" would have been considered significant.

2. Area measurement:

As discussed in the "Materials and Methods", a phantom study was conducted to check that the IVS in Winnipeg reported true areas, as outlined by the tracker ball, on a one to one basis. The six objects (four circles, $E$ and ) were measured on the actual phantom five times, their areas calculated and their resultant means and standard errors are reported in Table 9 as actual phantom areas. The same areas were measured, using the IVS, on five repeated standard phantom scans and these means and standard errors are reported in Table 9 as measured phantom scans. The difference between the actual phantom areas and those obtained from measurement on the IVS range from +0.46 square centimeters to -0.07 square centimeters and was considered to be small. The circles showed larger standard errors than the $E$ and because the $E$ and were outlined more accurately 
Table 8. Results of Mixed Analysis of Variance of CT numbers for muscle as reported by the Independent Viewing System (IVS) in Winnipeg and Edmonton for each slice of the same 5 subjects.

\begin{tabular}{llc}
\hline Source of Variation & df $^{*}$ & Mean Squares of Variables \\
\hline Among subjects & 4 & 2305.8809 \\
Among slices within subjects & 20 & 411.2610 \\
Between locations & 1 & 0.0549 \\
Among subjects by location & 4 & 3.7558 \\
Among slices within subjects & 20 & 1.5872 \\
$\quad$ by location & & \\
\hline
\end{tabular}

* degrees of freedom 
Table 9. Means and standard errors obtained for Independent Viewing System (IVS) measured phantom areas and actual phantom areas and the difference.

\begin{tabular}{|c|c|c|c|}
\hline & $\begin{array}{c}\text { Measured Phantam } \\
\text { Areas }\left(\mathrm{cm}^{2}\right)\end{array}$ & $\begin{array}{l}\text { Actual Phantom } \\
\text { Areas (cm. }{ }^{2} \text { ) }\end{array}$ & $\begin{array}{c}\text { Difference } \\
\text { (Meas.- Act.) }\end{array}$ \\
\hline Circle \# 1 & $16.06 \pm 0.11$ & $15.60 \pm 0.02$ & $+0.46 \pm 0.11$ \\
\hline Circle \# 2 & $20.18 \pm 0.10$ & $19.83 \pm 0.04$ & $+0.35 \pm 0.11$ \\
\hline Circle \# 3 & $20.28 \pm 0.12$ & $20.35 \pm 0.02$ & $-0.07 \pm 0.12$ \\
\hline Circle \#4 & $\begin{array}{r}20.00 \pm 0.14 \\
2.18 \pm 0.08 \\
2.36 \pm 0.04\end{array}$ & $\begin{array}{r}19.99 \pm 0.02 \\
2.20 \pm 0.01 \\
2.37 \pm 0.01\end{array}$ & $\begin{array}{l}+0.01 \pm 0.14 \\
-0.02 \pm 0.08 \\
-0.01 \pm 0.04\end{array}$ \\
\hline
\end{tabular}


than the circles using the IVS. This is because the boundaries of the $E$ and are straight edges and pixels are arranged in rows and columns. Outlining the circles using the IVS occurs in a stepwise fashion due to the pixels. Therefore, parts of the circles were excluded from the IVS area measurements. Hence, differences between actual and measured areasfor the circles show larger standard errors. As explained previously, areas measured for high resolution scans, using the IVS in Winnipeg, were measured as if they were standard scans. A phantom study determined the magnification factor for high resolution scans to be 4.0 with a standard error of 0.05 . Therefore all areas measured on high resolution scans, using the IVS in Winnipeg, were divided by a factor of four to obtain the corrected areas.

Using a paired "t" test, statistical comparison was performed between 1) areas measurements computed in Winnipeg, using its IVS, for each slice of the tongue scan series for 5 subjects and 2) area measurements computed in Edmonton, using its IVS, for the same slices of the tongue scan series for the same 5 subjects. This was done to determine the systematic error or bias between the IVS in Winnipeg and in Edmonton. The mean difference between the IVS equipment in the two locations was 0.0868 with a standard error of 0.0797 which is not different significantly from zero. Therefore, area measurements computed in Winnipeg are not different significantly from those computed in Edmonton and measurements from the 15 subjects measured in Winnipeg and the 22 subjects measured in Edmonton are assumed comparable. All 
subjects therefore can be treated as a group.

\section{Cadaver measurement:}

Four tongue scan series were conducted for one cadaver head and three tongue scan series for two cadaver heads. Tongue areas were measured and tongue volumes were computed, using the IVS and volume formula respectively. The means and standard errors of these tongue scan series for each cadaver tongue are reported in Table 10 as the "calculated" tongue volumes. Since subject tongue scan series could not be repeated more than once due to increased radiation exposure, these "calculated" cadaver tongue volumes reflect the effect that varying head positions may have upon volume measurement. As seen from Table 10, the standard errors for each cadaver tongue are small, ranging from \pm 1.08 to \pm 1.53 cubic centimeters. Therefore, the error in volumes due to head positioning differences from subject to subject was small and did not compromise the usefulness of intersubject comparisons. These "calculated" cadaver tongue volumes were compared to volumes obtained from the three cadaver tongues after dissection and repeated measurement by fluid displacement as described previously. The means and standard errors are reported for the dissected tongues as "actual" values in Table 10 and the percentage difference from "calculated" values also reported. The smallest percentage difference between "calculated" and "actual" tongue volumes is -1.17 percent for Cadaver 1, while the largest is -10.41 percent for Cadaver 2, with Cadaver 3 somewhere in between with a percentage difference of +4.49 percent. Part of the reason that the percentage difference is higher 
Table 10. Means and standard errors for calculated cadaver tongue volumes and actual cadaver tongue volumes and the difference.

\begin{tabular}{ccccc}
\hline & $\begin{array}{c}\text { Calculated }\left(\mathrm{cm}^{3}\right) \\
\text { (Cal.) }\end{array}$ & $\begin{array}{c}\text { Actual }\left(\mathrm{cm}^{3}{ }^{3}\right) \\
\text { (Act.) }\end{array}$ & $\begin{array}{c}\text { Difference } \\
\left(\mathrm{cm}^{3}\right)^{3} \\
\text { (Cal.-Act.) }\end{array}$ & $\begin{array}{c}\text { Difference } \\
(\%) \\
\text { (Cal./Act.) }\end{array}$ \\
Cadaver 1 & $119.31 \pm 1.08$ & $120.72 \pm 0.88$ & $-1.41 \pm 1.39$ & -1.17 \\
Cadaver 2 & $45.36 \pm 1.47$ & $50.63 \pm 0.48$ & $-5.27 \pm 1.55$ & -10.41 \\
Cadaver 3 & $70.45 \pm 1.53$ & $67.42 \pm 0.84$ & $+3.03 \pm 1.75$ & +4.49 \\
\hline
\end{tabular}


for Cadaver 2 than for 1 and 3 is mathematical as it is the smallest tongue of the three and a difference of only a few cubic centimeters between "calculated" and "actual" tongue volumes represents a larger percentage difference than for a difference of a similar number of cubic centimeters for a larger tongue. The mean tongue volume for subjects in this study was 59.12 cubic centimeters with a range of 42.63 to 84.50 cubic centimeters. Tongue and oral volumes for the subjects in this study are presented in Table 14 (Appendix VIII).

\section{B) Investigator Error:}

Pilot Study 1, as discussed in "Materials and Methods" (Section L), was conducted to test the measurement error involved in boundary identification of the tongue and the areas measured according to these boundaries using the IVS in Winnipeg and Edmonton. Pilot Study 2 dealt with measurement error involved in the location and measurement of study model variables, while Pilot study 3 dealt with measurement error of linear and angular cephalometric variables. However, only Pilot Study I will be discussed here in the "Results" since this aspect pertains directly to developing a technique by which to measure tongue volume relative to oral volume and tests the reliability of this investigator in identifying tongue boundaries consistently. Model and cephalometric error studies are not new methodologies. The maximum error committed 99 percent of the time during boundary identification and area measurement was 0.63 square centimeters in Winnipeg 
and 1.98 square centimeters in Edmonton (see Table 4 in "Materials and Methods"). Investigator error was larger in Edmonton, since the IVS in Edmonton rounded areas off to whole numbers, rather than reporting to one decimal place as in Winnipeg, and therefore more repetitions were required to establish the error. These results show that tongue boundaries in any particular slice can be identified consistently for subject tongue scan series.

For subsequent volumes obtained from area measurements of a series of slices, the error of measurement associated with each volume may be calculated. For subjects with an average number of 9 slices, the volume calculated was associated with a maximum error, 99 percent of the time, of 0.21 cubic centimeters for subjects measured in Winnipeg and 0.66 cubic centimeters for subjects measured in Edmonton. The measurement error associated with volumes calculated from area measurements of slices other than 9 are listed in Table 5. These results indicate that volume errors are small.

C) Results of Statistical Analysis:

1. Results of Student's "tt" test:

After subjecting the data to a Student's "t" test, the results of which are listed in Tables 11,12 and 13 (Appendix VII), the following significant differences were found for $\frac{\text { tongue volume }}{\text { oral volume }}$, cephalometric variables and model variables between the open-bite (O.B.) 
and nonopen-bite (N.O.B.) groups.

\section{i. Tongue Volume \\ Oral Volume}

The anterior open-bite subjects had a larger mean ratio of $\frac{\text { tongue volume }}{\text { oral volume }}(90.91 \pm 0.53)$ than that for nonopen-bite subjects $(86.03 \pm 0.69)$, which was significantly different at $p<0.01$. In other words the proportion of tongue relative to the space available for the tongue was significantly larger for open-bite subjects. Therefore, open-bite subjects had less space or oral volume within which to accomodate the tongue. For individual subject ratios, see Table 14 (Appendix VIII).

ii. Cephalometric Variables:

a) Intermaxillary Ht., Lth. and Area, Tongue and Hyoid: None of the means for measurements listed under this heading in Table 12 were significantly different between the O.B. and N.O.B. groups, except Ant. I.M. Ht. which was significantly larger for $0 . B$. subjects $(6.40 \pm$ $0.16)$ as compared to N.O.B. subjects $(5.79 \pm 0.07)$.

b) Cranial Base: No significant differences were found between O.B. and N.O.B. subjects for cranial base cephalometric variables.

c) Maxilla to Cranium: The mean for the angle SN-FH was significantly larger $(p<0.05)$ for $0 . B$. subjects $(9.01 \pm 1.44)$ as opposed to N.0.B. subjects $(5.51 \pm 0.65)$. The mean SNA angle was significantly smaller 
at $p=0.01$ for $0 . B$. subjects $(77.24 \pm 1.15)$ than that for N.0.B. subjects $(82.10 \pm 0.71)$. The means for angles SN-OP and SN-FOP were signficantly larger $(p<0.01)$ for $0 . B$. subjects $(20.65 \pm 1.70 ; 18.31 \pm$ 1.39) versus N.O.B. subjects $(14.59 \pm 0.86 ; 15.86 \pm 0.96)$. There was no significant difference between these two groups for SN-UOP.

d) Mandible to Cranium: The SNB angle was smaller for $0 . B$. subjects $(73.37 \pm 2.13)$ than that for N.0.B. subjects $(77.92 \pm 0.76)$ at $p<$ 0.05 . The mean for angle SN-LOP was significantly larger $(p<0.01)$ in O.B. subjects $(21.79 \pm 2.56)$ as compared to N.0.B. $\operatorname{subjects}(8.55 \pm$ 1.06). The mean $Y$ axis angle was significantly larger $(p<0.05)$ for O.B. subjects $(66.20 \pm 2.09)$ as opposed to N.0.B. subjects $(61.65 \pm$ 0.70). The means for the angles SN-LBMe, SN-GoGn and SN-GoMe (all representing commonly used mandibular plane angles) were all significantly larger $(p<0.01)$ for $0 . B$. subjects $(45.68 \pm 3.84 ; 43.97 \pm$ $3.77 ; \quad 45.89 \pm 3.65)$ than those for N.0.B. subjects $(31.57 \pm 1.24$; $31.01 \pm 1.14 ; 32.80 \pm 1.13)$. Also the gonial angle was significantly larger $(p<0.01)$ for $0 . B$. subjects $(129.48 \pm 3.26)$ as opposed to N.0.B. subjects $(121.56 \pm 1.03)$.

e) Mandible to Maxilla: The means for the angles OP-LBMe, OP-GoGn and OP-GoMe were all significantly larger $(p<0.01)$ for $0 . B$. subjects $(25.03 \pm 2.36 ; 23.32 \pm 2.30 ; 25.24 \pm 2.21)$ than those for N.0.B. subjects $(16.98 \pm 0.83 ; 16.42 \pm 0.73 ; 18.21 \pm 0.72)$. Similarly the means for angles UOP-LBMe, UOP-GoGn and UOP-GoMe were also significantly larger $(p<0.01)$ for $0 . B$. subjects $(27.37 \pm 2.49 ; 25.66 \pm$ $2.41 ; 27.60 \pm 2.29)$ as compared to N.0.B. subjects $(14.54 \pm 0.98$; 
$13.98 \pm 0.90 ; 15.77 \pm 0.88)$. On the other hand, the means for the angles LOP-LBMe, LOP-GOGn, LOP-GOMe and FOP-LBMe, FOP-GOGn, FOP-GoMe were not significantly different for the two groups. The means for the angles PP-LBMe, PP-GoGn and PP-GoMe were larger for O.B. subjects $(36.32 \pm 3.28 ; \quad 34.60 \pm 3.22 ; \quad 36.52 \pm 3.13)$ than those for N.O.B. subjects $(24.95 \pm 1.18 ; 24.38 \pm 1.09 ; 26.17 \pm 1.06)$ and were significant at $\mathrm{p}<0.01$. The mean $P P-O P$ angle is significantly larger $(p<$ $0.05)$ for $0 . B$. subjects $(11.29 \pm 1.35)$ compared to N.0.B. subjects $(7.97 \pm 0.61)$. The mean PP-LOP and PP-FOP angles are also larger for O.B. subjects $(12.42 \pm 2.08 ; 16.47 \pm 1.49)$ than those for N.0.B. subjects $(1.93 \pm 0.86 ; 9.23 \pm 0.79)$ but at a significance level of $\mathrm{p}<0.01$.

f) Dentoalveolar: None of the angular dentoalveolar cephalometric measurements listed in Table 12 were significantly different between open-bite and nonopen-bite groups. The linear measurements U.I to NA and U.I to AP were significantly larger $(p<0.05)$ for $0 . B$. subjects $(0.69 \pm 0.07 ; 0.97 \pm 0.13)$ as opposed to N.0.B. subjects $(0.41 \pm 0.05 ;$ $0.63 \pm 0.06)$. The linear distance $U .6$ to PP was larger for $0 . B$. subjects $(2.32 \pm 0.08)$ than that for N.0.B. subjects $(2.08 \pm 0.04)$ which was significant at $p<0.05$. On the other hand, the distances 0.6 to $\mathrm{PP} \perp \mathrm{OP}$ and $\mathrm{U} .6$ to $\mathrm{PP} \perp \mathrm{FOP}$ were also larger for $0 . \mathrm{B}$. subjects $(2.37 \pm$ $0.09 ; 2.42 \pm 0.09)$ versus N.0.B. subjects $(2.10 \pm 0.04 ; 2.11 \pm 0.04)$ but were significantly different at $p<0.01$. The distance 0.6 to $\mathrm{PP} \perp$ UOP was also larger for $0 . \mathrm{B}$. subjects $(1.64 \pm 0.07)$ than that for N.0.B. subjects $(1.50 \pm 0.03)$ but at a significance level of $p<0.05$. 
Although the distance U.I to $\mathrm{SN}$ and U.I to PP were both larger for the 0.B. group, the difference was not signficant from the N.0.B. group. The linear measure L.I to AP was significantly larger $(p<0.05)$ for O.B. subjects $(0.36 \pm 0.12)$ as compared to N.0.B. subjects $(0.08 \pm$ 0.04). No significant differences were found betwen the two groups for the perpendicular distances of L.I and L.6 to the three mandibular planes (LBMe, GoGn, GoMe).

g) Vertical Proportions: Both the mean TAFH and mean LAFH were significantly larger $(p<0.01)$ for $0 . B$. subjects $(11.57 \pm 0.28 ; 6.80 \pm$ $0.24)$ as those for N.0.B. subjects $(10.74 \pm 0.10 ; 6.04 \pm 0.08)$. No significant difference was found between the mean UAFH for the two groups but the O.B. group had larger UAFH than the N.O.B. group. Although $\mathrm{PFH}$ was found to be smaller for $0 . B$. subjects, it was not significantly different from N.O.B. subjects. The ratio $\frac{U A F H}{L A F H}$ was significantly smaller $(p<0.05)$ for $0 . B$. subjects $(0.70 \pm 0.03)$ relative to N.O.B. subjects $(0.78 \pm 0.01)$.

iii. Model Variables:

None of the means for model variables, as listed in Table 13 , were different significantly between the open-bite and nonopen-bite groups, except for the variable overbite. The mean overbite for the open-bite group was $-1.86 \pm 0.59$ as compared to the nonopen-bite group, where the mean was $4.13 \pm 0.34$. 
2. Results of Correlations:

Correlation coefficients between $\frac{\text { tongue volume }}{\text { oral volume }}$ and each of the cephalometric and model variables were calculated from all the subject data. Upon examination of these correlation coefficients, it was found that out of ninety-eight correlation coefficients calculated, only five were significant at $p<0.05$, indicating the nonexistence of a relationship between $\frac{\text { tongue volume }}{\text { oral volume }}$ and each of the variables studied. It is also recognized that since these correlation coefficients were calculated from all the data (O.B. group and N.O.B. group), they are "global" (Burdick andd Chebib, 1972) and hence no great value can be attached to them. 
Discussion

This study was undertaken with the primary intent of developing a more accurate technique than those used in the past, by which tongue volume could be measured, not only in absolute terms, but more importantly, relative to the space available for the tongue. In addition, ratios of $\frac{\text { tongue volume }}{\text { oral volume }}$ were calculated for open-bite versus nonopen-bite subjects and correlations of this ratio were made with dental and craniofacial dimensions. The tongue has been implicated in the etiology of dental malocclusion and obvious clinical examples are collapse or expansion of the dental arches in cases of congenital aglossia and macroglossia. However these cases are relatively rare. More common are those in which aberrations of tongue size are less obvious and not detectable by current methods. More important than the absolute size of the tongue, is its volume relative to the space within which it is contained. Where space is adequate within the oral cavity and oropharynx, the tongue will be accomodated. However, there may be several reasons why the tongue cannot be contained within the oral space. The first would be that the tongue is large physically while the space for it is of normal dimensions. Secondly, the space within the oral cavity and oropharynx may be small physically, as in mandibular micrognathia, while the tongue is of normal size. In other instances, enlarged and inflamed tonsils within the oropharynx may simply "crowd out" the tongue. Various combinations of the above may 
be present at one time. Inadequate space for the tongue may also be due to a differential in growth between the tongue and mandible, that is, most of tongue growth is complete before the mandible and it is not until pubertal and postpubertal growth that the mandible must "catch up" and complete its growth. During this period of disproportionate growth, the tongue must be positioned relatively high and forward within the oral cavity until mandibular growth catches up and creates more room for the tongue.

Tongue Measurement:

The relationship of tongue size to the space available for it within the oral cavity and oropharynx and how this relationship affects facial form and occlusion are of current clinical interest. This investigator felt that the use of computed tomography (CT) would be the most appropriate way to attack the problem of studying tongue size relative to its space. CT allows viewing of a selected crosssection of the body or head, without interference from adjacent tissues as with conventional radiographic tomography. By analyzing a series of contiguous slices of the tongue, it should be possible to assess the three-dimensional relationship of the tongue in vivo. Other studies attempting to measure tongue size have been less successful. Vig and Cohen (1974) and Cohen and Vig (1976) presented a two-dimensional method of measuring midsagittal tongue area and intermaxillary space area using tracings of lateral cephalographs. The obvious disadvantage of this technique is that the third dimension is ignored and 
there is no acceptable way of assessing how this dimension contributes to the actual size of the tongue or surrounding space. CT does take into account the third dimension and for this reason this investigator feels it is superior to other two-dimensional methods.

In view of the problem of the third dimension, Bandy (1966), Bandy and Hunter (1969) and Takada et al. (1980) attempted to measure tongue volume. Bandy (1966) and Bandy and Hunter (1969) developed a system of fluid displacement, using a mouthpiece into which the tongue was extended, while Takada et al. (1980) took alginate impressions of the tongue in its most protruded position, together with the lower dentition. Both of these studies were limited by the extent to which the tongue could be protruded into the measuring device or impression material. Only the anterior part of the tongue, at most the anterior two-thirds to the level of the circumvallate papillae could be measured. Although an effort was made to standardize tongue protrusion for each subject in the fluid displacement study, the amount of protrusion varied from subject to subject. There was no way of ensuring that the same proportion of tongue was protruded by each subject, i.e. no posterior or inferior limits were defined. Therefore, comparison of partial tongue volumes between individuals and relating these partial volumes to dental arch measurements are inappropriate. Although Takada et al. (1980) tried also to standardize the posterior and inferior limits of the tongue and the posterior limit of the oral cavity for the purpose of intersubject comparisons, again, only the volume of the anterior portion of the tongue was measured. The posterior and 
inferior tongue portions, which are significant, were excluded. The space for the tongue was evaluated only in the oral cavity proper and did not include the oropharynx.

While the anterior part of the tongue is important for functions such as speech and manipulation of the bolus during chewing, it is the dorsum of the tongue which interacts with the airway. As the base of the tongue forms the anterior wall of the pharyngeal airway, its position in posture is determined principally by factors regulating airway maintenance. This postural function of maintaining a patent pharyngeal airway takes precedence over other oropharyngeal functions (Bosma, 1963b). This in turn must affect the maturation and development of the surrounding skeleton and occlusion (Bosma, 1963a). Therefore previous studies which ignored this critical posterior aspect of the tongue, in attempting to measure volume (Bandy, 1966; Bandy and Hunter, 1969; Takada et al., 1980) exclude its airway function. Since current thinking relates growth of the jaws and position of the teeth to postural forces of the tongue, correlations between anterior tongue and malocclusion are irrelevant. Comparisons of tongue volume made to the volume of the oral cavity proper only are also irrelevant. With the advent of CT and the ability to obtain multiple transverse sequential slices of the tongue, the volume of the tongue between slices can be estimated and the size of the whole tongue calculated. It is unnecessary to exclude any major portion of the tongue with this technique, since slices can be commenced at the level of the hard palate above the tongue and continued downward to include all of 
the lowermost portion of the tongue. Anteroposteriorly, no part of the tongue will be excluded since the field size of high resolution scans can accomodate adequately the entire length of the tongue, the dental arches, oropharynx, prevertebral muscles and part of the vertebrae. Therefore, the tongue in its entirety can be included in a series of closely spaced slices for a subject and an assessment of tongue volume obtained. Also, the surrounding space for the tongue in both the oral cavity and oropharynx are included in these same slices and an assessment of oral volume can be obtained.

Tongue Boundaries:

This investigator found generally tongue boundaries in the uppermost and lowermost slices of a tongue series less difficult to determine than midregion slices. By establishing where the soft palate, uvula, temporalis muscle, pharyngeal muscles and oropharynx bounded the tongue, posterior and posterior lateral boundaries of the tongue were defined consistently from subject to subject for uppermost slices. This was done by visual examination of the images directly on the video screen of the Independent Viewing System (IVS) and with the aid of coronal and sagittal reconstructions. As explained in "Materials and Methods", a series of transverse slices could be reconstructed in coronal or sagittal planes as indicated by the cross-hair cursor lines over the selected transverse slice. Along with the bright-up marker which was used and which appeared at the same point in the transverse, coronal or sagittal mode, boundaries were defined 
and verified. Laterally and anteriorly, the teeth, alveolar process and hard palate bounded the tongue clearly. Any air space in the oral cavity proper was seen easily, either anterior to the tongue tip, as a border around the tongue at the height of contour or centrally where the tongue was curled up at the sides with a trough in the middle. Transition zones between air and tongue were seen at the edges of the tongue.

The tongue boundaries in lowermost slices were less difficult to determine than expected. The inferior limit of the tongue was defined as the last slice in which the genioglossus muscle appeared prior to the appearance of the geniohyoid muscle. As verified through the study of anatomical texts, texts of correlative anatomy with CT scans and the cadaver dissections performed in this study, the geniohyoid muscle had wider muscle bellies than the genioglossus and could be differentiated in this way. Also, at the level of the geniohyoid muscle, the hyoglossus and styloglossus muscles were less clear or not seen at all and less of the body of the mandible was seen anteriorly. The two bellies of the genioglossus muscle were usually seen for their full length in two or sometimes three slices, depending on the thickness of the muscle for that subject. Here, the tongue took on a characteristic pear shape, its lateral, posterior and posterior lateral boundaries defined by the sublingual gland, submandibular gland, mylohyoid muscle, epiglottis and oropharynx. From subject to subject, the areas varied in magnitude, but the pear shape of the tongue and appearance of the genioglossus and geniohyoid muscles were consistent 
features.

If an extra slice was measured at the lowermost level, due to mistaking the geniohyoid muscle for the genioglossus, the affect would

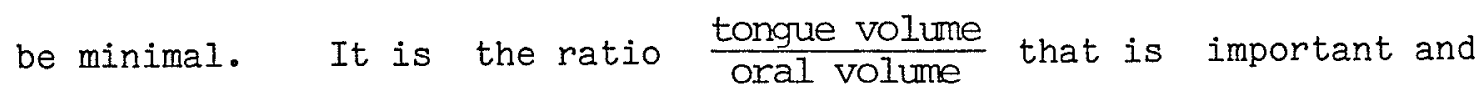
since the last two slices of a series are often similar in area (tongue and space area), the overall ratio is not affected greatly. It was at the uppermost level that should a slice be missed and the

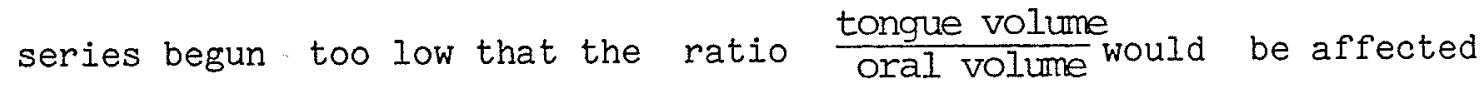
more seriously. This is due to the fact that if more space was present relative to the tongue, it was found usually in the uppermost slices. For instance, within the oral cavity, space might be seen anterior to the tongue tip, as a halo around the height of contour of the tongue or centrally in the trough of the tongue. Or the oropharynx might vary in size due to the angle at which the soft palate dropped. In this study, only one subject's tongue scan series was excluded since the first slice was begun too low and it was felt that the ratio obtained could not be compared reliably to others. In the lower levels of the tongue scan series, oral space was seldom seen and indicated that the tongue filled completely the space available for it in the floor of the mouth.

In midregion slices, anterior and lateral boundaries bounded by the teeth and alveolus, or posterior boundaries bounded by the oropharynx were relatively easy to identify; but posterior lateral boundaries were more difficult. In these slices sometimes a clear differentiation between the tongue and the muscles of the pharynx 
could not be made and sagittal reconstructions were not always helpful. A hint of temporalis or mylohyoid muscle, depending on the slice, was identified, usually at the buttressed portion of the mandible. A posterior lateral boundary could be identified as running laterally from the oropharynx to the buttressed portion of the mandible in a smooth curvature. This determination was based on subject scans where these boundaries were clearer and scans of cadavers with subsequent dissections.

Unlike previous studies, the entire tongue, including the critical posterior aspect, was measured in three dimensions and a volume measurement was obtained. Anterior, lateral, posterior, posterior lateral, upper and lower limits were defined consistently for each subject as indicated by the error study carried out on tongue boundary identification. Maximum error committed 99 percent of the time for any slice of a series during tongue boundary identification and area measurement was 0.63 square centimeters in Winnipeg and 1.98 square centimeters in Edmonton. Virtually no aspect of the tongue was excluded therefore intersubject comparisons are valid. Also a comparison of tongue volume to oral volume was appropriate, since oral space was measured also for each subject and was measured for every slice in which the tongue appeared. Boundaries of the space around the tongue were more easily defined than for the tongue itself; the only difficulty being the transiton zones between air and soft tissue, such boundaries being midlined. 
Accuracy of Volume Measurement with $\mathrm{CT}$ :

Volume measurement of the tongue appears to be feasible with CT. This is true also for oropharyngeal volume. Montgomery et al. (1979) derived volumes for the nasal airways of four cadaver heads from a series of transverse CT images and compared these to the volumes of silicone rubber casts of the same cadaver nasal airways. They found an overall percentage difference of one percent between CT-derived volume determinations and volumes of the casts. Lipton et al. (1978) used CT to obtain volume measurements of postmortem silastic casts of the human left ventricle and compared the results with those obtained by biplane radiography. The true volume of the casts was obtained using Archimedes' principle and was the basis for comparison of the two techniques. CT measurements were found to be significantly more accurate and precise. The goal of both studies was to test the accuracy of CT-derived volume determinations as a necessary step in the application of $\mathrm{CT}$ to similar volume measurements in vivo. This study deals mainly with in vivo determination of tongue and oral volumes. This is considerably more difficult, since these in vivo determinations result from dynamic systems and not static cadavers or casts. Also landmarks and boundaries, especially of the tongue, are more difficult to establish and require an in depth knowledge of the anatomy of the area. This knowledge was gained by the study of texts of anatomy and CT correlations with cross-sectional anatomy, along with cadaver dissections. The study, investigating nasal airway volumes, 
consisted of bony landmarks of the nasal wall and turbinates. Although nasal mucosa makes up a small zone of intermediate density between the bony structures and air of the cavity, boundary identification is still relatively easy. Also, silastic ventricular casts placed in a plexiglass shell for positioning on the scanner will show reasonably definite edges of the silastic next to the air within the plexiglass container, even though cardiac ventricles are irregular in shape and trabeculated.

In this study, the boundaries of the oral spaces were identified relatively easily since the blackness due to air contrasts clearly with that of soft tissue. Where there were transition zones between air and soft tissue, boundaries were midlined. Where soft tissue boundaries must be determined, similar problems, as those encountered in this study, will occur in other in vivo studies. This is especially so where soft tissues differ little in density from each other. However, as CT has the ability to demonstrate small differences in $x$-ray attenuation of tissues, it may be better than any other modality for determining soft tissue boundaries. This problem of soft tissue boundary identification, although present, does not detract from the usefulness and accuracy of CT-derived volumes. It is at present the best technique, in this researcher's opinion, for deriving volumes. In this particular study, the advantage of being able to measure the entire tongue along with its surrounding space outweighs the difficulties encountered in the determination of the soft tissue boundaries.

In this study, the actual tongue volumes were compared to CT cal- 
culated volumes for three cadaver tongues. The differences between the two (Cal. - Act.) were $-1.41,-5.27$ and +3.03 cubic centimeters. While a sample of three is not enough to establish a mean difference between actual and CT-derived volumes, it does give an indication of how closely to the actual volumes the CT-derived volumes come for a structure such as the tongue.

Methods of Measuring Areas on CT Scans:

Montgomery et al. (1979) measured nasal airways from tracings of enlarged one to one photographs of each slice or tomogram. Lipton et al. (1978) derived ventricular cast volumes by contour drawings on pixel prints, that is, a computer printout of each slice. Both methods are acceptable. However, in this study, measurements were made directly on the original image as viewed on the video screen of the IVS.

In particular, use of the computer printout in this study, would have been extremely tedious and time consuming, considering the number of subjects and slices involved. Lipton et al. (1978) found attenuation numbers fairly uniform in matrix elements definitely within or outside the cast and the contour line was averaged between the two. In this study, it was easier to visualize structures on the actual CT image, rather than viewing a computer printout of a 320 x 320 matrix for each slice. The edge of the tongue was difficult to define on a computer printout due to the nonuniformity of CT numbers. 
Tensing or Contraction of the Tongue During Scans:

Is the volume of the tongue different when it is tensed or contracted as compared to when it is in a relaxed state? It has been shown for a living muscle cell, that over a wide range of muscle lengths, the actin and myosin filament lattice maintains a constant volume i.e. muscle contraction is isovolumetric at the cellular level (Elliott et al., 1963). However, when considering muscle tissue, such as a biceps muscle, contraction of skeletal muscle mechanically affects muscle blood flow and therefore, blood volume within the muscle. The contracting muscle fibers exert external pressure on the vasculature, extruding blood from capillaries and veins, and impeding inflow from the arteries. From work done on dogs, muscle blood volume was found to be relatively low at 2.0 to 3.0 milliliters of blood per 100 grams of resting muscle (Lesh and Rothe, 1969). The average weights of the dissected cadaver tongues were 122.3, 68.1 and 48.7 grams. On the basis of Lesh and Rothe (1969), if the blood volume is decreased 60 percent during contraction, expected reductions in tongue volumes would be $2.2, \quad 1.2$ and 0.66 milliliters respectively. As the tongue is said to be well vascularized, these reductions in volumes are expected to be somewhat greater. It was not possible to determine if the tongue was tensed in subjects in this study. However, all subjects appeared at ease during tongue examinations. 
Motion Artifact, High-Attenuation Differential Artifact, Positional Changes of Tongue and Head Between Scans:

The main causes of artifacts in a CT scan are motion during the scan and large differences of attenuation values of adjacent tissues (Gonzalez et al., 1976). Motion during a scan was minimized with good cooperation and head support. Motion of a point during a scan places that point in different computed positions during the scanning cycle. This false representation produces a linear artifact called a "streak". The head can rotate in all directions, thus producing a variety of streaking outside or inside the cranial vault. Aside from head movement, which is minimal with good head support, tongue movement during the scan can be responsible also for artifact. The subjects in this study were trained prior to the tongue scan series to hold the tongue in a stable position without swallowing during each scan; to swallow between scans; and then to return the tongue to that stable position for the next scan. If a subject did swallow during a scan, it was obvious when viewing the image on the video screen. Consequently that particular scan was repeated and the bad scan deleted from the series. Fortunately this occurred rarely. Movement due to respiration is a problem when scanning the body (thorax or abdomen) but did not seem to present a problem during these tongue scan series. A small amount of tongue or head movement occurring while scans were taking place was not detectable. Any streaking which occurred could not be differentiated as resulting from minor movements rather than 
from large differences in attenuation values at the interfaces of adjacent tissues to be discussed next.

Streaking occurs also at interfaces of adjacent tissues which have markedly different linear attenuation coefficients, such as airbone or bone-soft tissue interfaces. The computer "overloads" due to the high differential values and produces the streak. This type of streaking is most noticible when scanning through the crowns of teeth, particularly if metallic restorations are present. However, the most severe streaking due to teeth or metallic restorations usually occurred through the body of the tongue and not at the upper or lowermost slices. Anterior, lateral and posterior boundaries in midregion slices (teeth, alveolar process, oropharynx) could still be identified if streaking was present. If parts of the posterior lateral boundaries of the tongue were involved, extrapolation of the boundary between streaks was necessary. Streaking artifact in this study was due mainly to bone-soft tissue or tooth-soft tissue interfaces rather than motion and could not be avoided. However, according to the error study undertaken to investigate the accuracy of tongue boundary identification (pages 75 and 92 ), area measurements were not affected seriously.

Another problem of movement was that which occurred between, rather than during, each scan or slice, i.e. the tongue was not held in the same stable position from scan to scan during a series. Positional changes, that were noticed from scan to scan when conducting tongue scan series, usually occurred between the initial slices. This 
was probably because the subject was not yet accustomed to the procedure. If this were the case, those one or two slices were repeated. This was necessary in less than 6 subjects. Obvious positional changes of the tongue missed during the scanning procedure, would have been noticed also when examining photographs of the scan series after the procedure. Fortunately, no such positional changes were found and it was not necessary to discard a scan series for this reason. A complete scan series would not have been repeated if changes in tongue position were found later. In addition, excessive head movement (usually sideways), which occurred between scans, could be seen on the video screen at the time of the procedure or when viewing photographs of the series later. However, in this study, it was found that such movement occurred at the end of a series, probably as the subject was tiring. Fortunately, such movement occurred in scans below the level of the genioglossus muscle and did not include areas that required to be measured. Therefore no scan series were discarded for this reason. No scan series with serious positional changes of the tongue or head between each scan were included in this study. If minor positional changes occurred of either the tongue or head between scans, this was not detectable. It was hoped that instruction and training of subjects prior to the procedure would minimize positional changes of the tongue. The head support minimized movement of the head, both during and between scans.

Technical errors could be responsible also for artifacts. Occurrence of these errors would be recognized by the x-ray technician con- 
ducting the scan series.

Varying Intersubject Head Positions:

Although precautions were taken to keep head position standardized from subject to subject for the tongue scan series, it was expected that variations would occur from subject to subject. However the repeated cadaver scan series showed that the standard errors of total tongue volumes due to head positioning variability were small, ranging from \pm 1.08 to \pm 1.53 cubic centimeters. As long as attempts were made to include the complete tongue in each tongue scan series for each subject, minor head variation from subject to subject should not compromise the usefulness of intersubject comparisons. That is, beginning each tongue scan series above or including the height of the contour of the tongue in the first slice and continuing down to the level of the hyoid bone would include virtually all of the tongue and its surrounding space. If tongue scan series where taken such that significant portions of the tongue or surrounding space were missed, for example at the upper or lower ends of a scan series, then variations in head position would have a more pronounced effect on calculated volumes and ultimately affect the ratio of $\frac{\text { tongue volume }}{\text { oral volume }}$. Therefore in this study care was taken to include the entire tongue. When examining photographs of tongue scan series after the scanning procedure, one series was discarded for the reason that the initial slice was begun at too low a level and excluded portions of tongue and oral space. 
Scan Increments:

Slices or scans in this study were conducted at 5.0 millimeter increments. To increase the accuracy of derived tongue and oral volumes, increments could have been decreased to 3.0 millimeters for instance. However, this increase in accuracy had to be weighed against the concomitant increase in radiation that would occur as a result of additional slices. Lipton et al. (1978) calculated volumes of ventricular casts from scan series taken at $0.5,1.0,1.5$ and 2.0 centimeter increments. They found with spacing up to 1.5 centimeters, there was no significant increase in the variance of volume measurements, while spacing of 2.0 centimeters resulted in significant errors. Therefore if increments of 0.5 to 1.5 centimeters do not significantly affect volume calculations for ventricular casts, a change of 0.5 to 0.3 centimeter increments for a study such as this could be assumed not to produce more accurate volumes.

$\frac{\text { Tongue Volume }}{\text { Oral Volume }}$ - O. B. Versus N. O. B. :

While the primary purpose of this study was to develop a technique for accurately quantifying the size of the tongue and its surrounding space, considering that the forward posturing of the tongue may be involved in the causation of anterior open-bite malocclusion, it was desirable to examine the ratio $\frac{\text { tongue volume }}{\text { oral volume }}$ of anterior 
open-bite subjects as compared to that of the rest of the sample, to examine if a difference in this ratio existed between the two groups.

Although the sample of anterior open-bite (O.B.) subjects was

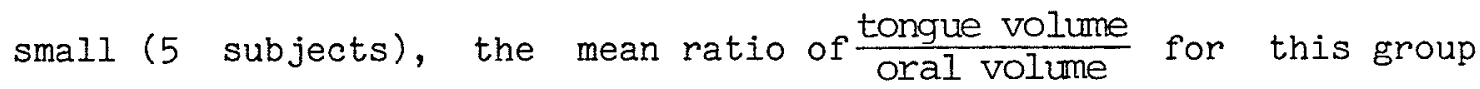
$(90.91 \pm 0.53)$ was larger than for the nonopen-bite (N.0.B.) group $(86.03 \pm 0.69)$ and was significantly different at $p<0.01$. This means that for these open-bite subjects, the proportion of tongue relative to oral volume was sigificantly greater than for non-open bite subjects. Possibly in these open-bite subjects the disparity in size between tongue volume and oral volume is great enought that the tongue cannot be contained within the oral cavity and oropharynx without impinging on the airway. Hence the compensatory mechanism seems to be to hinge open the mandible, posture the tongue forward and create an anteror open-bite as a result of tonic forward posturing of the tongue, rather than other compensatory mechanisms occurring as discussed in "Review of the Literature". The significantly larger ratio

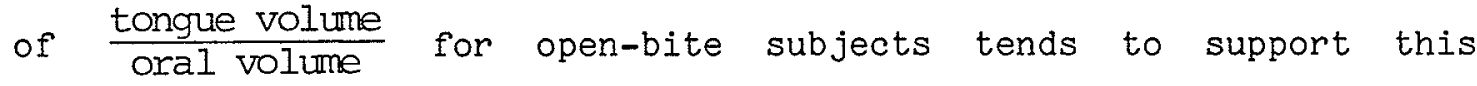
hypothesis. The open-bite subjects in this study ranged in age from 12 years 9 months to 20 years 3 months with a mean of 16 years 1 month. It might be expected to see higher ratios in younger subjects, since it is prior to the pubertal growth spurt that a disparity in tongue volume relative to oral volume is more likely to exist due to differential growth of the mandible and tongue. However this openbite sample also included subjects past their pubertal growth spurt, indicating that a disproportion of tongue volume to oral volume still 
persisted.

$\underline{\text { Cephalometric Variables }}=\underline{0} \cdot \underline{B} \cdot \underline{\text { Versus }} \underline{N} \cdot \underline{0} \cdot \underline{B} \cdot:$

Previous investigators (Subtelny and Sakuda, 1964, Isaacson et al., 1971; Nahoum, 1971, 1975; Nahoum et al., 1972; Lowe, 1980) have studied various craniofacial variables of anterior open-bite subjects and compared them to subjects who did not have anterior open-bites. This investigator also examined many of the same variables in order to compare the open-bite sample of this study with other anterior openbite samples.

The cephalometric results reported in this study for $0 . B$. subjects as opposed to N.O.B. subjects are in general agreement with these previous investigators. A more detailed discussion of these results than that which follows can be found in Appendix IX. Steep occlusal plane angles were found in this study as in other studies. The maxilla (SNA), although comparable in size (ANS-PNS, MxUL) for both groups in this study, was found to be retropositioned in $0 . \mathrm{B}$. subjects here as in other studies, except for Lowe (1980), who found it to be positioned within normal limits. In O.B. subjects, a distortion of the maxilla is often evident, as the premaxilla seems to tip up anteriorly towards the base of the skull giving the impression that the premaxillary area did not grow vertically. However Nahoum (1971) was the only investigator to find the SN-PP angle significantly smaller in $0 . B$. subjects $(p<0.001)$.

Differences were found in the shape and position of the mandible 
between the two groups in this study. In agreement with other studies, the mandible (SNB) was found to be retruded relative to the cranial base. Steep mandibular plane angles, larger gonial angles and larger $Y$ axis angles were also common findings in the $O . B$. groups of the various studies, indicating a backward rotation of the mandible.

Other studies (Subtelny and Sakuda, 1964; Lowe, 1980) have found larger spatial discrepancies between the mandible and maxilla (ANB angle), while this study did not. This may be because their studies compared O.B. subjects to normal occlusions, whereas in this study, the N.O.B. subjects included Class I, Class II and Class III malocclusions. As in other studies, the divergence of the mandible to the maxilla was indicated by larger PP-mandibular plane angles and larger OP-mandibular plane angles.

In this study, both upper and lower first permanent molars and permanent incisors were positioned similarly for 0.B. and N.O.B. subjects, except for overeruption of the maxillary first permanent molar. This agrees with Subtelny and Sakuda (1964) except they also found greater vertical development of the upper incisor. Nahoum et al. (1972) and Nahoum (1975) either found normal or undererupted maxillary incisors and decreased dentoalveolar height in the region of the mandibular first molar. Overeruption of the maxillary first molar, as seen in this study, may contribute to the backward rotation of the mandible. Or is this overeruption of the maxillary molar secondary to an opening rotation of the mandible, as demonstrated by Lowe et al. (1977) and Lowe (1978), creating an anterior open-bite? The normal 
intermittent forces of occlusion would be expected to maintain the vertical position of the teeth, but possibly because of changes in muscle length due to the opening rotation of the mandible, there is decreased muscle tension which allows the posterior teeth to erupt in anterior open-bite malocclusions. Proffit (1978) has cited work which suggests that a reduction in biting force, which allows more eruption of posterior teeth, is one factor in producing an anterior open-bite. Aside from steep mandibular and occlusal plane angles in O.B. subjects, an opening rotation of the mandible would also be reflected in increased anterior face height. This study, along with Subtelny and Sakuda (1964) and Isaacson et al. ( 1971), found greater TAFH and LAFH in O.B. subjects, while UAFH was similar to N.O.B. subjects. This resulted in a ratio of $\frac{U A F H}{\mathrm{LAFH}}$ which was significantly smaller in O.B. subjects. Lowe (1980) found no significant difference between $0 . B$. and N.O.B. subjects for UAFH, LAFH and TAFH. Lowe feels that his findings, in contrast to others, may reflect the fact that Class I skeletal open-bites are composed of two subgroups, with one subgroup exhibiting considerably larger LAFH and TAFH.

Posterior face height (Go-S or Go $\perp$ SN) has been found to be shorter in O.B. subjects in other studies. This study found a trend to decreased $\mathrm{PFH}$ but not at a level of significance.

Model Variables $=\underline{0} \cdot \underline{B} \cdot$ Versus $\underline{N} \cdot \underline{0} \cdot \underline{B} \cdot:$

Model variables were also studied in this investigation, but none were found to be significant between the two groups except overbite. 
The 0.B. group had a mean overbite of $-1.86 \pm 0.59$ which was significantly different $(p<0.01)$ from the N.O.B. group which had a mean overbite of $4.13 \pm 0.34$.

Clinical and Research Implications of $\mathrm{CT}$ :

Computed tomography offers the best method to date to assess hard and soft tissues in three dimensions in vivo. In particular, it is presently the most accurate method by which to obtain a measure of tongue volume and oral volume. This is important for those patients suspected of having a discrepancy between tongue volume and oral volume, as there is now a way of determining the extent of this discrepancy prior to attempting extensive orthodontic treatment, which could be doomed to relapse.

As the anterior open-bite sample in this study is small, future skeletal anterior open-bite patients seen at the Graduate Orthodontic Clinic at the University of Manitoba should have a tongue scan series conducted, similar to the manner described in this study, as part of the initial record taking procedure. In this way, a larger sample of anterior open-bite subjects with appropriate records can be collected.

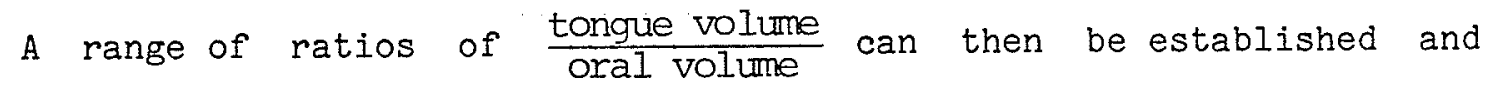
related to the degree of anterior open-bite and other craniofacial dimensions. Such a study would help determine which patients are amenable to orthodontic treatment alone with no or minimal relapse and which patients require a combination of orthodontic treatment and surgical intervention to correct the malocclusion. In anterior open-bite 
patients who have undergone orthognathic surgery, it is not known how the ratio $\frac{\text { tongue volume }}{\text { oral volume }}$ is affected. Therefore using CT to evaluate such patients before and after surgery would be extremely useful.

Evaluation of other types of orthognathic surgery, such as for Class II and Class III malocclusions, could also be undertaken with the use of CT and bone and soft tissue relationships studied both before and after surgery. In addition, Becker et al. (1976) have suggested the use of $\mathrm{CT}$ for patients with craniofacial malformations, who are to undergo orthognathic surgery, in order to evaluate periorbital muscles, optic nerves, major blood vessels and any individual variation which may be present in such patients. CT as an adjunct for treatment planning of reconstructive procedures could be extended to cleft lip and cleft palate patients, facial trauma patients and evaluating the thickness of the mandibular ramus in patients with small mandibles requiring ramal procedures (Treacher-Collins Syndrome).

There is currently interest in evaluating the soft tissue components of the temperomandibular joint (TMJ), with the use of CT, which is a noninvasive technique, rather than with arthrography, which is an invasive technique (Baker, 1981b). An initial trial procedure shows this to be possible and will be more fully investigated in a future study

Montgomery et al. (1979) have suggested that patients for whom nasal surgery is being planned to improve respiration can be evaluated with CT. CT evaluation of the nasal airway, along with measurement of nasal airflow, could help predict whether the planned surgery will. 
succeed in improving nasal respiration.

The size of adenoid tissue and the degree of nasal blockage could also be more accurately assessed using CT and compared to the lateral cephalograph which is presently used to assess the size of the adenoid tissue.

Aside from using $\mathrm{CT}$ in order to discover pathology, which is its prime purpose at present, its role could be expanded to include other areas of research and clinical application in dentistry as just discussed. 
Summary and Conclusions

The tongue has been held generally to play a dominant role in the control of tooth position. Current clinical interest has focused on the abnormal posturing of the tongue as an etiologic factor in the causation of malocclusion. In addition, aside from obvious clinical examples, whether aberrations of tongue size relative to oropharyngeal space exist, cannot be detected by current methods. Past attempts made at measuring tongue size have not been adequate as major portions of the tongue, especially the posterior aspect, have been excluded. The primary purpose of this investigation was to develop a more accurate technique than those used in the past, by which tongue volume could be measured relative to its surrounding space. With the use of computed tomography (CT), this objective appeared to be possible.

Using CT, a series of sequential transverse plane scans or slices of 5.0 millimeter increments were obtained for 32 subjects, area measurement of the tongue and oral space carried out for each slice of each subject's tongue scan series using the Independent Viewing System (IVS), and tongue and oral volumes calculated using a mathematical formula. Subsequent ratios of $\frac{\text { tongue volume }}{\text { oral volume }}$ were correlated to various cephalometric and model variables for all subjects and $\frac{\text { tongue volume }}{\text { oral volume }}$, cephalometric and model measurements compared for anterior open-bite $\left(\mathrm{O}_{\bullet} \mathrm{B}_{\bullet}\right)$ subjects versus nonopen-bite $\left(\mathrm{N}_{\bullet} \mathrm{O}_{\bullet} \mathrm{B}_{\bullet}\right)$ subjects. From analysis of the data, the following conclusions emerged. 1. Measurement of the complete tongue and its surrounding space to 
obtain tongue and oral volumes is indeed possible using CT and provides the most accurate method at present for assessing the three-dimensional relationship of the tongue and oropharyngeal space.

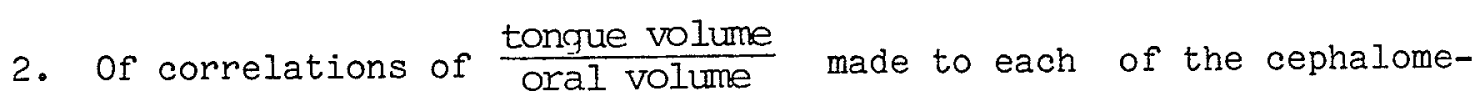
tric and model variables, only five of ninety-eight were significant at $p<0.05$, indicating that no relationship existed between $\frac{\text { tongue volume }}{\text { oral volume }}$ and each of the variables studied for the group as a whole.

3. The ratio tongue volume was found to be significantly larger $(p<0.01)$ for anterior 0.B. subjects than for N.0.B. subjects, indicating a greater proportion of tongue relative to oral volume was present in the O.B. subjects in this sample.

4. Cephalometric results for the O.B. versus N.O.B. group were found to be in general agreement with other previous investigators. O.B. subjects as compared to N.O.B. subjects exhibited:

i) steeper occlusal plane angles

ii) steeper mandibular plane angles

iii) larger palatal plane - mandibular plane angles

iv) larger occlusal plane - mandibular plane angles

v) larger gonial angles

vi) larger $Y$ axis angles

vii) retropositioned maxilla and mandible

viii) greater eruption of the maxillary first permanent molar

ix) increased total and lower anterior face height.

5. Model variables investigated were found not to be significantly different between $O . B$. and N.O.B. groups, except for the variable 
overbite.

The use of CT may be extended to other areas of research and clinical application rather than primarily to discover pathology. It is recommended that future skeletal anterior open-bite patients seen at the Graduate Orthodontic Clinic at the University of Manitoba, have a tongue scan series conducted, in order to collect a larger sample of open-bite and to establish a range of ratios $\frac{\text { tongue volume }}{\text { oral volume }}$ and correlate these with the degree of anterior open-bite and other craniofacial dimensions. 


\section{Bibliography}

Abelson, S.M., Brodie, A.G., Bronstein, I.P. and Schreiber, S.L. (1941). Muscular macroglossia. Am. J. Dis. Child. 62: $624-628$.

Alderisio, J.P. (1954). A preliminary report on the forces about the dentition. (Abstr.) Am. J. Orthod. 40: 234-235

Alderisio, J.P. and Lahr, R. (1953). An electronic technique for recording the myodynamic forces of the lip, cheek and tongue. J. Dent. Res. 32: 548-553.

Baker, C.G. (1981a). Personal communication.

Baker, C.G. (1981b). Personal communication.

Baker, R.E. (1954). Tongue and dental function. Am. J. Orthod. 40: 927-939.

Ballard, C.F. (1953). The significance of soft tissue morphology in diagnosis, prognosis and treatment planning. Trans. Eur. Orthod. Soc. pp. 143-169.

Ballard, C.F. (1957). The aetiology of malocclusion - an assessment. Dent. Pract. Dent. Rec. 8: 42-51.

Bandy, H.E. (1966). A method of studying tongue volume. M. Sc. Thesis, University of Michigan, Ann Arbor.

Bandy, H.E. and Hunter, W.S. (1969). Tongue volume and the mandibular dentition. Am. J. Orthod. 56: 134-142.

Becker, M.H., McCarthy, J.G., Chase, N., Converse, J.M. and Genieser, N.B. (1976). Computerized axial tomography of craniofacial malformations. Am. J. Dis. Child. 130: 17-20.

Bjuggren, G., Jensen, R. and Strombeck. J.O. (1968). Macroglossia and 
its surgical treatment. Scand. J. Plast. Reconstr. Surg. 2: $116-124$.

Boese, L.R. (1969). Increased stability of orthodontically rotated teeth following gingivectomy in Macaca nemestrina. Am. J. Orthod. 56: 273-290.

Bosma, J.F. (1963a). Maturation of function of the oral and pharyngeal region. Am. J. Orthod. 49: 94-104.

Bosma, J. (1963b). Oral and pharyngeal development and function. J. Dent. Res. 42: 375-380.

Boyd, E. (1980). Origins of the Study of Human Growth, University of Oregon Health Sciences Center Foundation, Ch. 34.

Brain, W.E. (1969). The effect of surgical transsection of free gingival fibers on the regression of orthodontically rotated teeth in the dog. Am. J. Orthod. 55: 50-70.

Brash, J.C., McKeag, H.T.A. and Scott, J.H. (1956). The Aetiology of Irregularity and Malocclusion of the Teeth, The Dental Board of the United Kingdom, London, pp. $\overline{199}-2 \overline{02,}$ 207-217, 438-441.

Briggs, C.P. (1965). Congenital aglossia and congenital cerebro-facial palsy (Moebius' Syndrome): Two contrasting cases. Trans. Eur. Orthod. Soc. pp. 169-179.

Brodie, A.G. (1950). Anatomy and physiology of head and neck musculature. Am. J. Orthod. 36: 831-844.

Brodie, A.G. (1952). Consideration of musculature in diagnosis, treatment and retention. Am. J. Orthod. 38: 823-835.

Brodie, A.G. (1957). Thoughts on the aetiology of malocclusion. Trans. Eur. Orthod. Soc. pp. 200-215.

Brodie, A.G. (1971). Emerging concepts of facial growth. Angle Orthod. 41: 103-118. 
Brooks, R.A. and Di Chiro, G. (1975). Theory of image reconstruction in computed tomography. Radiology 117: 561-572.

Burdick, J.A. and Chebib, F.S. (1972). Inter- and intrasubject correlations of sleep data. J. Gen. Psychol. 87: 259-276.

Campbell, P.M., Moore, J.W. and Matthews, J.L. (1975). Orthodontically corrected midline diastemas; A histological study and surgical procedure. Am. J. Orthod. 67: 139-158.

Carter, B.L., Morehead, J., Wolpert, S.M., Hammerschlag, S.B., Griffiths, H.J. and Kahn, P.C. (1977). Cross-Sectional Anatomy. Computed Tomography and Ultrasound Correlation, Appleton-CenturyCrofts, New York.

Chebib, F.S. and Burdick, J.A. (1973). Estimation of measurement error. J. Gen. Psychol. 89: 47-58.

Chebib, F.S., Cleall, J.F. and Carpenter, K.J. (1976). On-line computer system for the analysis of cephalometric radiographs. Angle Orthod. 46: 305-311.

Christensen, E.E., Curry, III, T.S. and Dowdey, J.E. (1978). An Introduction to the Physies of Diagnostic Radiology, Lea and Febiger, Philadelphia.

Cleall, J.F. (1965). Deglutition: A study of form and function. Am. J. Orthod. 5I: 566-594.

Cleall, J.F. and Chebib, F.S. (1971). Coordinate analysis applied to orthodontic studies. Angle Orthod. 41: 214-218.

Cohen, A.M. and Vig, P.S. (1976). A serial growth study of the tongue and inter-maxillary space. Angle Orthod. 46: 332-337.

Cormack, A.M. (1963). Representation of a function by its line integrals, with some radiological applications. J. Appl. Phys. 34 : 2722-2727. 
Dixon, D.A. (1960). An investigation into the influence of the soft tissues on tooth position. Dent. Pract. Dent. Rec. 10: 89-92.

Edwards, J.G. (1968). A study of the periodontium during orthodontic rotation of teeth. Am. J. Orthod. 54: 441-461.

Edwards, J.G. (1970). A surgical procedure to eliminate rotational relapse. Am. J. Orthod. 57: 35-46.

Egyedi, P. (1965). Reduction of tongue size in the surgical correction of jaw deformity. Br. J. Oral Surg. 3: 13-21.

Elliott, G.F., Lowy, J. and Worthington, C.R. (1963). An x-ray and light diffraction study of the filament lattice of striated muscle in the living state and in rigor. J. Mol. Biol. $\underline{6}$ : $295-305$.

Eskew, H.A., Charlotte, N.C. and Shepard, E.E. (1949). Congenital aglossia. Am. J. Orthod. 35: 116-119.

Falford, G.E., Ardran, G.M. and Kemp. F.H. (1956). Aglossia congenita. Arch. Dis. Child. 31: 400-407.

Gardiner, J.H. (1960). Congenital partial aglossia. Dent. Pract. Dent. Rec. 10: 83-87.

Gonzalez, C.F., Grossman, C.B. and Palacios, E. (1976). Computed Brain and Orbital Tomography, Technique and Interpretation, John Wiley and Sons, New York.

Gould, M.S.E. and Picton, D.C.A. (1962). A method of measuring forces acting on the teeth from the lips, cheeks and tongue. Br. Dent. J. 112: 235-242.

Gould, M.S.E. and Picton, D.C.A. (1964). A study of pressures exerted by the lips and cheeks on the teeth of subjects with normal occlusion. Arch. Oral Biol. 9: 469-478.

Gould, M.S.E. and Picton, D.C.A. (1968). A study of pressures exerted 
by the lips and cheeks on the teeth of subjects with Angle's Class II Division 1, Class II Division 2 and Class III malocclusions compared with those of subjects with normal occlusions. Arch. Oral Biol. 13: 527-541.

Graber, T.M. (1963). The "three M's": Muscles, malformation, and malocclusion. Am. J. Orthod. 49: 418-450.

Graber, T.M. (1966). Orthodontics = Principles and Practice, W.B. Saunders Co, Philadelphia, pp. $3 \overline{17}, 319,28 \overline{4-2} 85$.

Gwynne-Evans, E. and Tulley, W.J. (1956). Clinical types. Dent. Pract. Dent. Rec. 6: 222-235.

Harvold, E.P. (1968). The role of function in the etiology and treatment of malocclusion. Am. J. Orthod. 54: 883-898.

Harvold, E.P. and Vargervik, K. (1971). Morphogenetic response to activator treatment. Am. J. Orthod. 60: 478-490.

Harvold, E.P. Vargervik, K. and Chierici, G. (1973). Primate experiments on oral sensation and dental malocclusions. Am. J. Orthod. 63: $494-508$.

Harvold, E.P. and Tomer, B.S. Primate experiments on muscle function in jaw and dental arch morphogenesis. In preparation, as cited by Vargervik, K. (1979). Morphologic evidence of muscle influence on dental arch width. Am. J. Orthod. 76: 21-28.

Hopkin, G.B. (1967). Neonatal and adult tongue dimensions. Angle Orthod. 37: 132-133.

Hounsfield, G.N. (1973). Computerized transverse axial scanning (tomography): Part 1. Description of system. $\mathrm{Br} . \mathrm{J}$. Radiol. 46: 1016-1022.

Hovell, J.H. (1955). Recent advances in orthodontics. Br. J. Orthod. 98: $114-122$. 
Hovell, J.H. (1962). The relation of the oro-facial musculature to occlusion: Current British thought. In Vistas in Orthodontics, Kraus, B.S. and Reidel, R.A. (Eds.), Lea and Febiger, Philadephia, pp. 328-345.

Isaacson, J.R., Isaacson, R.J., Speidel, T.M. and Worms, F.W. (1971). Extreme variation in vertical facial growth and associated variation in skeletal and dental relations. Angle Orthod. 4l: 219-229.

Kelly, J.R., Sorenson, H.W. and Turner, E.G. (1978). Prosthodontic treatment for Pierre Robin syndrome. J. Prosthet. Dent. 39: $554-560$.

Köle, H. (1965). Results, experience, and problems in the operative treatment of anomalies with reverse overbite (mandibular protrusion). Oral Surg. 19: 427-450.

Kuhl, D.E. and Edwards, R.Q. (1968). Reorganizing data from transverse section scans of the brain using digital processing. Radiology 91: 975-983.

Kuroda, T. and Ohyama, K. (198I). Hypoglossia: Case report and discussion. Am. J. Orthod. 79: 86-94.

Kydd, W.L. (1957). Maximum forces exerted on the dentition by the perioral and lingual musculature. J. Am. Dent. Assoc. 55: 646-65l.

Kydd, W.L., Akamine, J.S., Mendel, R.A. and Kraus, B.S. (1963). Tongue and lip forces exerted during deglutition in subjects with and without an anterior open bite. J. Dent. Res. 42: 858-866.

Lear, C.S.C. and Moorrees, C.F.A. (1964). Measurement of oro-facial muscle forces. (Abstr.) J. Dent. Res. 43: 906.

Lear, C.S.C., Catz, J., Grossman, R.C., Flanagan, J.B. and Moorrees, C.F.A. (1965a). Measurement of lateral muscle forces on the dental arches. Arch Oral Biol. 10: 669-689. 
Lear, C.S.C., Flanagan, J.B., Jr. and Moorrees, C.F.A. (1965b). The frequency of deglutition in man. Arch. Oral Biol. 10: 83-99.

Lear, C.S.C. and Moorrees, C.F.A. (1969). Buccolingual muscle force and dental arch form. Am. J. Orthod. 56: 379-393.

Lesh, T.A. and Rothe, C.F. (1969). Sympathetic and hemodynamic effects of capacitance vessels in dog skeletal muscle. Am. J. Physiol. 217: 819-827.

Linder-Aronson, S. (1970). Adenoids: Their effect on mode of breathing and nasal airflow and their relationship to characteristics of the facial skeleton and the dentition. Acta Otolaryngol. Suppl. 265: 1-132.

Linder-Aronson, S. (1975). Effects of adenoidectomy on the dentition and facial skeleton over a period of five years. In Transactions of the Third International Orthodontic Congress, J.T. Cook (Ed.), C.V. Mosby Co., Saint Louis.

Lipton, M.J., Hayashi, T.T., Boyd, D. and Carlsson, E. (1978). Measurement of left ventricular cast volume by computed tomography. Radiology 127: 419-423.

Lowe, A.A. (1978). Mandibular joint control of genioglossus muscle activity in the cat (Felis domesticus) and monkey (Macaca irus). Arch. Oral Biol. 23: 787-793.

Lowe, A.A. (1980). Correlations between orofacial muscle activity and craniofacial morphology in a sample of control and anterior open-bite subjects. Am. J. Orthod. 78: 89-97.

Lowe, A.A., Gurza, S.C. and Sessle, B.J. (1977). Regulation of genioglossus and masseter muscle activity in man. Arch. Oral Biol. 22: $579-584$.

Lowe, A.A. and Johnston, W.D. (1979). Tongue and jaw muscle activity in response to mandibular rotations in a sample of normal and anterior open-bite subjects. Am. J. Orthod. 76: 565-576. 
Magnusson, T.E. (1972). Congenital aglossia with aplasia of several teeth and multiple impactions. Trans. Eur. Orthod. Soc. pp. $517-526$.

Marshall, C.H. (1976). Principles of computed tomography. Postgrad. Med. 60: 105-109.

McCullough, E.C. (1977). Factors affecting the use of quantitative information from a CT scanner. Radiology 124: 99-107.

McCullough, E.C., Baker, H.L., Jr., Houser, O.W. and Reese, D.F. (1974). An evaluation of the quantitative and radiation features of a scanning x-ray transverse axial tomograph: The EMI scanner. Radiology 111: 709-715.

McCullough, E.C. and Payne, J.T. (1976). Performance evaluation: CT-scanners. Presented at the International Sumposium and Course on Computerized Tomography (CT), San Juan, Puerto Rico, as cited by Christensen, E.E., Curry, III, T.S. and Dowdey, J.E. (1978). An Introduction to the Physics of Diagnostic Radiology, Lea and Febiger, Philadelphia.

McCullough, E.C. and Payne, J.T., (1978). Patient dosage in computed tomography. Radiology 129: 457-463.

Moir, R.W. (1978). A cephalometric analysis of a North American Indian tribe: A cross sectional study. M. Sc. Thesis, University of Manitoba, Manitoba.

Montgomery, W.M., Vig, P.S., Staab, E.V. and Matteson, S.R. (1979). Computed tomography: A three-dimensional study of the nasal airway. Am. J. Orthod. 76: 363-375.

Nahoum, H.I. (1971). Vertical proportions and the palatal plane in anterior open-bite. Am. J. Orthod. 59: 273-282.

Nahoum, H.I. (1975). Anterior open-bite: A cephalometric analysis and suggested treatment procedures. Am. J. Orthod. 67: 513-521. 
Nahoum, H.I. Horowitz, S.L. and Benedicto, E.A. (1972). Varieties of anterior open-bite. Am. J. Orthod. 61: 486-492.

Naidich, T.P., Pudlowski, R.M., Leeds, N.E. and Deck, M.D.F. (1978). Case report: Hypoglossal palsy: Computed tomography demonstration of denervation hemiatrophy of the tongue associated with glomus jugulare tumor. J. Comp. Assist. Tomogr. 2: 630-632.

Oldendorf, W.H. (1961). Isolated flying spot detection of radiodensity discontinuities - Displaying the internal structural pattern of a complex object. IRE Trans. Bio-med. Electronics. 8 : $68-72$.

Petterson, G. (1961). Aglossia congenita with bony fusion of the jaws. Acta Chir. Scand. 122: 93-95.

Phelps, M.E., Hoffman, E.J. and Ter-Pogossian, M.M. (1975). Attenuation coefficients of various body tissues, fluids, and lesions at photon energies of 18 to $136 \mathrm{keV}$. Radiology 117: 573-583.

Popovich, F. and Thompson, G.W. (1977). Craniofacial templates for orthodontic case analysis. Am. J. Orthod. 71: 406-420.

Proffit, W.R. (1978). The facial musculature in its relation to the dental occlusion. In Muscle Adaptation in the Craniofacial Region, D.S. Carlson and J.A. McNamara (eds.), Monograph No. 8, Craniofacial Growth Series, Center of Human Growth and Development, The University of Michigan, Ann Arbor.

Proffit, W.R., Kydd, W.L., Wilskie, G.H. and Taylor, D.T. (1964). Intraoral pressures in a young adult group. J. Dent. Res. 43 : 555-562.

Proffit, W.R., McGlone, R.E. and Christiansen, R.L. (1965). Lingual pressures against anterior and lateral areas of the palate during speech and swallowing. IADR (Abstr.) pp. 59, no. 89.

Proffit, W.R., Chastain, B.B. and Norton, L.A. (1969). Linguopalatal pressure in children. Am. J. Orthod. 55: 154-166. 
Proffit, W.R. and Norton, L.A. (1970). The tongue and oral morphology: Influences of tongue activity during speech and swallowing. ASHA Reports, No. 5, pp. 106-115.

Proffit, W.R. and Mason, R.M. (1975a). Myofunctional therapy for tongue-thrusting: Background and recommendations. J. Am. Dent. Assoc. 90: 403-411.

Proffit, W.R., McGlone, R.E. and Barrett, M.J. (1975b). Lip and tongue pressures related to dental arch and oral cavity size in Australian aborigines. J. Dent. Res. 녀: 1161-1172.

Radon, J. (1917). On the determination of functions from their integrals along certain manifolds. Berichte uber die Verhandlungen der koniglich Sashsischen Gesellschaft der Wissenschaften $\mathrm{zu}$ Leipzig. Mathematisch-Physische Klasse 69: 262-277 (ger.), as cited by Brooks, R.A. and DiChiro, G. (1975). Theory of image reconstruction in computed tomography. Radiology 117: 561-572.

Reitan, K. (1959). Tissue rearrangement during retention of orthodontically rotated teeth. Angle Orthod. 29: 105-113.

Ricketts, R.M. (1954). The cranial base and soft structures in cleft palate speech and breathing. Plast. Reconstruct. Surg. I4: 47-61.

Ricketts, R.M. (1958a). The functional diagnosis of malocclusion. Trans. Eur. Orthod. Soc. pp. 42-67.

Ricketts, R.M. (1958b). Respiratory obstructions and their relation to tongue pressure. (Abstr.) Cleft Palate Bul. $\underline{8}$ : 4-5.

Ricketts, R.M. (1968). Respiratory obstruction syndrome. Am. J. Orthod. 54: 495-514.

Rix, R.E. (1953). Some observations upon the environment of the incisors. Dent. Rec. 73: 427-441.

Scott, J. (1961). The role of the soft tissues in determining normal and abnormal dental occlusion. Dent. Pract. Dent. Rec. 11: 
$302-308$.

Shear, M. (1956). Congenital underdevelopment of the maxilla associated with partial adactylia, partial anodontia and microglossia. J. Dent. Assoc. S. Afr. 11: 78-83.

Subtelny, J.D. and Sakuda, M. (1964). Open-bite: Diagnosis and treatment. Am. J. Orthod. 50: 337-358.

Subtelny, J.D. and Sakuda, M. (1966). Muscle function, oral malformation, and growth changes. Am. J. Orthod. 52: 495-517.

Takada, K., Sakuda, M., Yoshida, K. and Kawamura, Y. (1980). Relations between tongue volume and capacity of the oral cavity proper. J. Dent. Res. 59: 2026-2031.

Takagi, Y., Gamble, J.W. and Proffit, W.R. (I967). Postural change of the hyoid bone following osteotomy of the mandible. Oral Surg. 23: 688-692.

Ter-Pogossian, M.M. (1977). Computerized cranial tomography: Equipment and physics. Semin. Roentgenol. 12: 13-25.

Tomes, C.S. (1873). The bearing of the development of the jaws on irregularities. Dental Cosmos 15: 292-296.

Vargervik, K. (1979). Morphologic evidence of muscle influence on dental arch width. Am. J. Orthod. 76: 21-28.

Vasiga, G.J. (1972). Functional occlusal plane inclination and maxillary differential vertical first molar height in Class I, II, and III, twelve-year old females. Dipl. Orthod., University of Toronto, Toronto.

Vig, P.S. and Cohen, A.M. (1974). The size of the tongue and intermaxillary space. Angle Orthod. 44: 25-28.

Walker, D.G. (1962). The tooth, the bone, and the muscle. Dent. Pract. Dent. Rec. 12: 383-387. 
Wallen, T.R. (1974). Vertically directed forces and malocclusion: A new approach. J. Dent. Res. 53: 1015-1022.

Weinstein, S. (1967). Minimal forces in tooth movement. Am. J. Orthod. 53: 881-903.

Wickwire, N.A., White, R.P. and Proffit, W.R. (1972). The effect of mandibular osteotomy on tongue position. J. Oral Surg. 30: 184-190.

Winders, R.V. (1956). A study in the devlopment of an electronic technique to measure the forces exerted on the dentition by the perioral and lingual musculature. Am. J. Orthod. 42: 645-657.

Winders, R.V. (1958). Forces exerted on the dentition by the perioral and lingual musculature duirng swallowing. Angle orthod. 28: $226-235$.

Worms. F.W., Meskin, L.H. and Isaacson, R.J. (1971). Open-bite. Am. J. Orthod. 59: 584-595. 


\section{Parental Consent Form}

\section{Dear Parent:}

In the Orthodontic Department at the University of Manitoba, we are currently conducting a study attempting to measure the size of the tongue. We believe that a large tongue may be involved in causing some dental malocclusions ( $i$. e. crooked teeth) and may also be important as regards the stability of the teeth after orthodontic treatment.

We will be conducting tongue examinations at the Health Sciences Centre using a method called computed tomography (CT). The procedure is simple, painless and lasts about 15 minutes. The subject simply lies on the examining table while the CI machine rotates about the head and conducts the scan of the tongue. The information from the scans will a]. low us to reconstruct the size of the tongue. Examinations will be conducted either on Saturday mornings or early evenings and tongue examination will be done only once. The radiation due to this method is small and is comparable to the amount of radiation your child would receive from a full mouth survey (FMS) for dental examination purposes. ( CT - range 1 - 5 rads ; FMS - 4 - 6 rads ). Maximum lead protection will cover your child during the scan.

We would greatly appreciate your permitting your child to take part in this study. However it is understood that you may withdraw your child at any time. Would you please indicate below whether or not you wish your child to participate and return the form in the attached envelope.

Thank you very much.

I grant permission for my child in this project. to participate (insert child's name)

I do not grant permission for my child cipate in this project. (insert child's name) to parti- 
Appendix II

Correlation of $\mathrm{CT}$ number of Homogeneous Materials with Optical Density

Over a wide range of attenuation coefficients, the CT number is linearly related to the effective linear attenuation coefficient $(\mu)$ (McCullough et al., 1974). However this is only applicable, in its strictest sense, for the CT number that results from a homogeneous material. For objects that have varying composition, the CT number will represent an average attenuation coefficient. A phantom study was carried out to test if this linear relationship did exist for CT numbers of different homogeneous materials. The CT numbers for a water bath and four circular objects (Circles 1, 2, 3 and 4 on phantom - see Figure 9), each consisting of a different homogeneous material, were obtained for five different phantom scans, using the Independent Viewing System (IVS) as described in "Materials and Methods". X-ray photographs were taken of two of the phantom scans and the optical density measured at five different points within the region of the water bath and four circles on each photograph, using an optical densitometer* at the Health Sciences Centre. Plotting the mean CT number obtained from the water bath and each of the four circles versus their mean optical densities results in Graph I. A straight line representing a linear relationship between CT numbers and optical density can be drawn through the five points plotted. However the relationship is not exactly linear and the five points fall approximately equally on either side of the line, rather than on it. This represents errors 
resulting mainly from variations in $x$-ray images due to processing of the $x$-ray film.

* Macbeth Division of Kollmorgen Corp., Newburgh, N. Y., U. S. A. Model No. TD502 


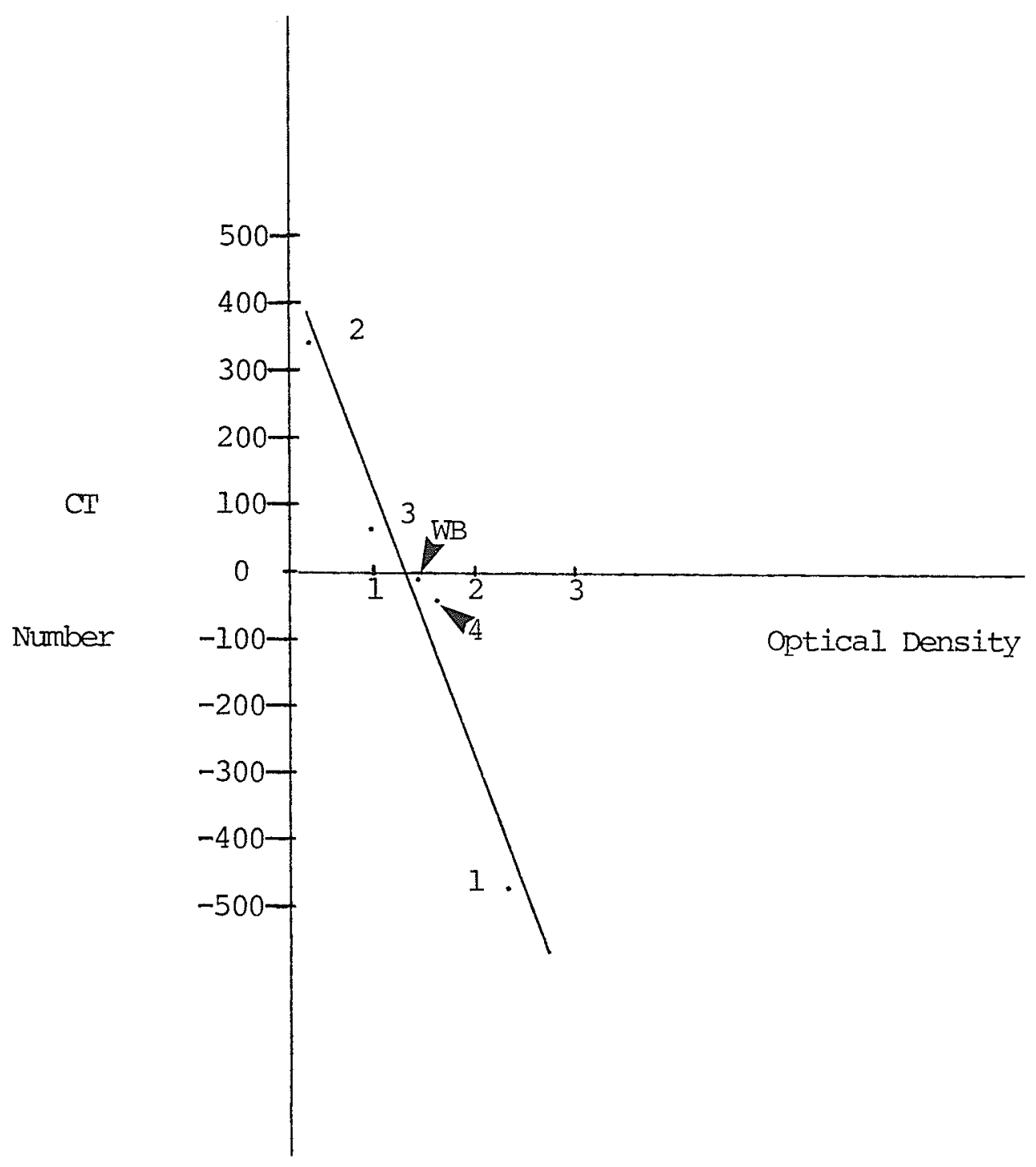

Graph 1. Plot of the CT number versus optical density for four different homogeneous materials (Circles 1, 2, 3 and 4 on the phantom) and a water bath (WB). 


\section{Formula for the Calculation of Volumes}

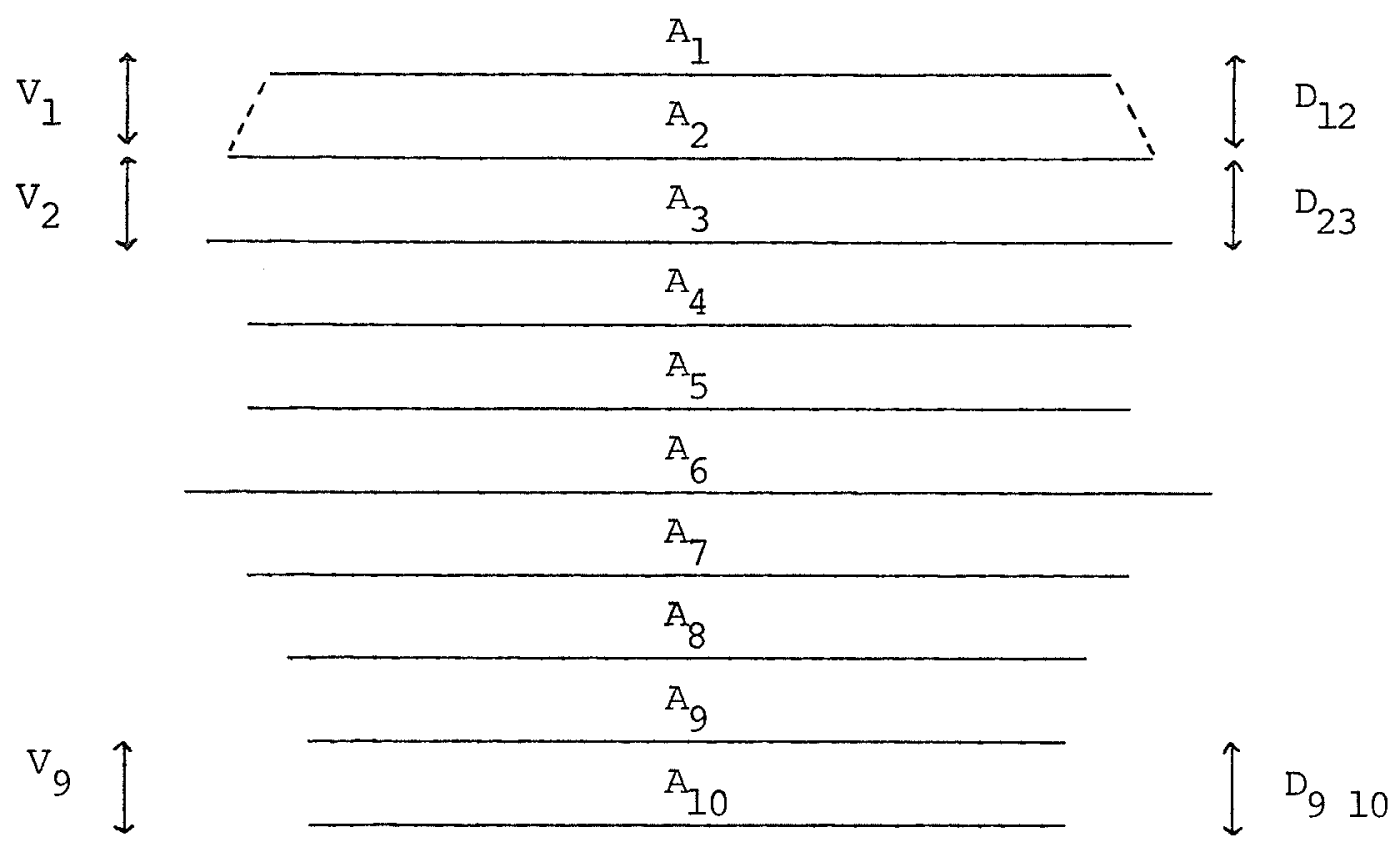

$$
\begin{aligned}
& V_{1}=\frac{A_{1}+A_{2}}{2} \quad D_{12} \\
& \text { where } V=\text { Volume } \\
& V_{2}=\frac{A_{2}+A_{3}}{2} \quad D_{23} \\
& \mathrm{~A}=\text { Area per } \\
& \text { slice } \\
& D=\text { Distance } \\
& V_{i}=\left[\frac{\left.A_{i}+A_{(i}+1\right)}{2}\right] D_{i} \\
& \text { between } \\
& \text { each slice } \\
& V_{i}=\frac{I}{2}\left[\sum A_{i} D_{i}+\sum A_{(i+I)} D_{i}\right] \\
& V=\frac{D}{2}\left[\sum A_{i}+\sum A_{(i+1)}\right] \\
& V=\frac{D}{2}\left[2 \sum_{2}^{n-I} A_{j}+A_{1}+A_{n}\right] \\
& \mathrm{i}=1, \mathrm{n}-1 \\
& i+I=2,2, n \\
& j=2, n-1
\end{aligned}
$$


Appendix IV

Definitions of Study Model Measurements

1. Overjet - Measurement in a horizontal plane between the labial aspect of the maxillary permanent central incisors and the labial surface of the mandibular permanent central incisors when the teeth are in centric occlusion. Where one maxillary central incisor was more labial to the other, both were measured and the average taken.

2. Overbite - The distance which the maxillary permanent central incisal margin closes vertically past the mandibular permanent central incisal margin when the teeth are in centric occlusion. Where one maxillary central incisor was longer than the other, both were measured and the average taken.

3. Open-bite - The distance between the incisal edges of the maxillary permenent central incisors and the incisal edges of the mandibular permanent central incisors in a vertical plane where there is lack of incisal overlap.

4. Arch Length - The distance between a line tangent to the incisors and a line tangent to the distal crown surfaces of the deciduous second molars or their permanent successors, the second premolars.

5. Intercanine Width - The distance between the canine cusp tips or when worn, between the centers of the resulting facets.

6. Intermolar Width - The distance between the mesio-lingual cusp tips of the permanent first molars.

7. Arch Circumference - The distance obtained by measuring the length of a curved soft brass wire over the buccal cusps or incisal edges of the teeth, from the distal surface of one deciduous second molar or permanent premolar to the distal surface of its antimere.

8. Palatal Vault Height - The distance in a vertical plane from the central fossae of the first permanent molars to the depth of 
the hard palate in the midline. 
Appendix $V$

Glossary of Cephalometric Landmarks and Planes

\section{Landmarks}

1. Sella (S) - The center of sella turcica as determined by inspection.

2. Nasion (N) - The midpoint of the frontonasal suture. Usually the point where the nasal suture meets the frontonasal suture.

3. Orbitale (Or) - The deepest point on the infraorbital margin of the bony orbit (bisected).

4. Anterior Nasal Spine (ANS) - The most anterior point on the maxilla at the level of the palate.

5. A point on the superior contour of the anterior nasal spine where the vertical thickness is 3.0 millimeters.

6. A point on the lower contour of the anterior nasal spine where the vertical thickness is 3.0 millimeters.

7. Subspinale ("A" point) - The deepest point on the midline contour of the alveolar process between the anterior nasal spine and the alveolar crest of the maxillary permanent central incisor.

8. A perpendicular line constructed from point "A" to the palatal plane and subsequently intersecting the bony upper contour of the anterior nasal spine region.

9. The apex of the maxillary permanent central incisor.

10. The incisal edge of the maxillary permanent central incisor (U.I).

11. The anterior point selected in order to construct the occlusal 
plane $(O P)$.

12. The incisal edge of the lower permanent central incisor (L.I).

13. The lingual shadow of the upper or lower permanent central incisor where it intersects the occlusal plane.

14. The apex of the mandibular permanent central incisor.

15. Supramentale ("B" point) - The deepest point on the midline contour of the mandible between pogonion and the alveolar crest of the mandibular permanent central incisor.

16. Pogonion $(\mathrm{Pg})$ - The most anterior point on the contour of the chin.

17. Gnathion (Gn) - The most anterior inferior point on the lateral shadow of the chin.

18. Menton (Me) - The lowest point on the symphyseal outline of the chin.

19. The most anterior point of the tongue.

20. The anterior point selected in order to construct the functional occlusal plane(FOP)-bisection of the vertical overlap of the first and second bicuspids.

21. Mesiobuccal cusp tip of the mandibular first permanent molar (L.6).

22. Mesiobuccal cusp tip of the maxillary first permanent molar (U.6).

23. Bisection of the vertical overlap of the maxillary and mandibular first permanent molars. 
24. Posterior Nasal Spine (PNS) - The most posterior point on the bony palate in the sagittal plane.

25. The most anterior inferior point on the body of the hyoid bone.

26. The most inferior point on the posterior one-third of the lower border of the mandible.

27. Gonion (Go) - The most posterior inferior point at the angle of the mandible.

28. Point where the occlusal plane (OP) intersects the posterior pharyngeal wall.

29. Basion ( $\mathrm{Ba}$ ) - The most anterior inferior point in the sagittal plane on the anterior rim of the foramen magnum.

30. Machine Porion (Po) - The top of the ear rods shadow representing the midpoint on the upper edge of the external auditory meati.

31. The most superior point on the head of the condyle.

32. Temporomandibular Joint (TMJ) - A point on the contour of the glenoid fossa indicating the maximum mandibular length as measured from prognathion (PGN).

33. Prognathion (PGN) - A point on the contour of the bony chin indicating maximum mandibular length as measured from TMJ.

\section{Planes}

A. Sella-Nasion (SN) - Constructed by a line joining sella and nasion $(1-2)$.

B. Frankfort Horizontal (FH) - Constructed by a line joining machine porion and orbitale $(30-3)$. 
C. Palatal Plane (PP) - Constructed by a line joining anterior nasal spine with posterior nasal spine (4-24).

D. Lower Occlusal Plane (LOP) - Constructed by a line joining the mesiobuccal cusp tip of the mandibular first permanent molar to the incisal edge of the mandibular permanent central incisor $(21-12)$.

E. Occlusal Plane (OP) - Constructed by a line bisecting the vertical overlap of the first permanent molars and the permanent central incisors $(23-11)$.

F. Functional Occlusal Plane (FOP) - Constructed by a line drawn through the bisection of the vertical overlap of the first permanent molars posteriorly and through the bisection of the vertical overlap of the first and second bicuspids anteriorly $(23-20)$.

G. Upper Occlusal Plane (UOP) - Constructed by a line joining the mesiobuccal cusp tip of the maxillary first permanent molar to the incisal edge of the maxillary permanent central incisor $(22-10)$.

H. Mandibular Plane - Gonion - Gnathion (GoGn) - Constructed by a line joining gonion and gnathion (27-17).

I. Mandibular Plane - Gonion - Menton (GoMe) - Constructed by a line joining gonion and menton $(27-18)$.

J. Mandibular Plane - Plane constructed by a line tangent to the posterior one-third of the lower border of the mandible and joining menton (LBMe, 26-18).

K. Facial Plane (FP) - Constructed by a line joining nasion and pogonion $(2-16)$. 
Appendix VI

Description of Linear and Angular Cephalometric Measurements

I Intermaxillary Ht. Lth. and Area, Tongue and Hyoid

Ant. I.M. Ht. (Anterior intermaxillary space height) - The length of a perpendicular from the palatal plane (4-24) to menton (18) (Vig and Cohen, 1974).

Post. I.M. Ht. (Posterior intermaxillary space height) - The length of a perpendicular from the palatal plane (4-24) to the mandibular plane (GoMe, 27-18) which passes through the point where the occlusal plane intersects the posterior pharyngeal wall (28) (Vig and Cohen, 1974).

I.M. Lth. (Length of the intermaxillary space) - Measured along the occlusal plane from the point where it intersects with the lingual shadow of the upper or lower incisor anteriorly, to where it cuts the pharyngeal wall posteriorly (13-28) (Vig and Cohen, 1974).

I.M. Area (Intermaxillary area index) $\frac{\text { Ant. I. M. Ht. + Post. I. M. Ht. }}{2} \quad X \quad$ I. M. Lth. (Vig and Cohen, 1974).

Vert. pos. of hyoid (Vertical position of the hyoid bone) - Length of a perpendicular from the palatal plane $(4-24)$ to the hyoid bone (25) (Cleall, 1965).

Hor. pos. of tongue (Horizontal position of the tongue tip) - Angle formed by the intersection of lines from the tongue tip (19) and the hyoid bone (25) both meeting at the upper bony border outline of the anterior nasal spine region (8).

II Cranial Base

SNBa - Angle formed by the intersection of the anterior cranial base 
(SN,1-2) with the posterior cranial base (SBa, 1-29).

SN - Length of the anterior cranial base from sella to nasion (1-2).

SBa - Length of the posterior cranial base from sella to basion $(1-29)$.

\section{Maxilla to}

SN-FH - Angle formed by the intersection of the anterior cranial base (SN, 1-2) with the Frankfort horizontal plane (FH, 30-3).

SNA - Angle formed by the intersection of the anterior cranial base (SN, 1-2) with a line joining "A" point to nasion (7-2).

$\mathrm{NA}-\mathrm{FH}$ - Angle formed by the intersection of a line joining "A" point to nasion (7-2) with the Frankfort horizontal plane (FH, 30-3).

Angle of Convexity (NAPg) - Angle formed by the intersection of a line joining nasion to "A" point $(2-7)$ with a line joinig "A" point to Pogonion $(7-16)$.

SN-OP - Angle formed by the intersection of the anterior cranial base (SN, 1-2) with the occlusal plane (OP, 23-11).

SN-UOP - Angle formed by the intersection of the anterior cranial base (SN, 1-2) with the upper occlusal plane (UOP, 22-10).

SN-FOP - Angle formed by the intersection of the anterior cranial base (SN, 1-2) with the functional occlusal plane (FOP, 23-20).

SN-PP - Angle formed by the intersection of the anterior cranial base (SN, 1-2) with the palatal plane (PP, 4-24).

ANS-PNS - Distance between anterior nasal spine (4) and posterior nasal spine (24) of the maxilla.

MxUL (Maxillary unit length) - Distance from temporomandibular joint 
(TMJ, 32) to a point on the lower contour of the anterior nasal spine where the vertical thickness is 3.0 millimeters (6).

IV Mandible to Cranium

Facial Angle - Inferior posterior angle formed by the intersection of the Frankfort horizontal (FH, 30-3) with the facial plane (FP, $2-16)$.

SNB - Angle formed by the intersection of the anterior cranial base (SN, 1-2) with a line joining "B" point with nasion (15-2).

SN-LOP - Angle formed by the intersection of the anterior cranial base (SN, 1-2) with the lower occlusal plane (LOP, 21-12).

$\mathrm{Y}$ axis - The anterior inferior angle where the Frankfort horizontal plane (FH, 30-3) is intersected by a line joining sella to gnathion $(1-17)$.

SN-GoGn - Angle formed by the intersection of the anterior cranial base (SN, 1-2) with the mandibular plane (GoGn, 27-17).

SN-GoMe - Angle formed by the intersection of the anterior cranial base (SN, 1-2) with the mandibular plane (GoMe, 27-18).

SN-LBMe - Angle formed by the intersection of the anterior cranial base (SN, 1-2) with the mandibular plane (LBMe, 26-18).

Gonial Angle - Angle formed by the intersection of a line joining the superior aspect of the condyle with gonion (sup. condyle Go, 31-27) with the mandibular plane (GoGn, 27-17).

Corpus Lth. - Distance from gonion (27) to gnathion (17) of the body of the mandible.

MdUL (Mandibular unit length) - Distance from temporomandibular joint (TMJ, 32) to prognathion (PGN, 33). 
$\checkmark$ Mandible to Maxilla

ANB - Angle formed by the intersection of the lines joining "A" point with nasion (7-2) and "B" point with nasion (15-2).

OP-GoGn - Angle formed by the intersection of the occlusal plane (OP, 23-11) with the mandibular plane (GoGn, 27-17).

OP-GoMe - Angle formed by the intersection of the occlusal plane (OP, 23-11) with the mandibular plane (GoMe, 27-18).

OP-LBMe - Angle formed by the intersection of the occlusal plane (OP, 23-11) with the mandibular plane (LBMe, 26-18).

UOP-GoGn - Angle formed by the intersection of the upper occlusal plane (UOP, 22-10) with the mandibular plane (GoGn, 27-17).

UOP-GOMe - Angle formed by the intersection of the upper occlusal plane (UOP, 22-10) with the mandibular plane (GoMe, 27-18).

UOP-LBMe - Angle formed by the intersection of the upper occlusal plane (UOP, 22-10) with the mandibular plane (LBMe, 26-18).

LOP-GoGn - Angle formed by the intersection of the lower occlusal plane (LOP, 21-12) with the mandibular plane (GoGn, 27-17).

LOP-GoMe - Angle formed by the intersection of the lower occlusal plane (LOP, 21-12) with the mandibular plane (GoMe, 27-18).

LOP-LBMe - Angle formed by the intersection of the lower occlusal plane (LOP, 2l-12) with the mandibular plane (LBMe, 26-18).

FOP-GoGn - Angle formed by the intersection of the functional occlusal plane (FOP, 23-20) with the mandibular plane (GoGn, 27-17).

FOP-GoMe - Angle formed by the intersection of the functional occlusal plane (FOP, 23-20) with the mandibular plane (GoMe, 27-18). 
FOP-LBMe - Angle formed by the intersection of the functional occlusal plane (FOP, 23-20) with the mandibular plane (LBMe, $26-18$ ).

PP-GoGn - Angle formed by the intersection of the palatal plane (PP, 4-24) with the mandibular plane (GoGn, 27-17).

PP-GoMe - Angle formed by the intersection of the palatal plane (PP, 4-24) with the mandibular plane (GoMe, 27-18).

PP-LBMe - Angle formed by the intersection of the palatal plane (PP, 4-24) with the mandibular plane (LBMe, 26-18).

PP-OP - Angle formed by the intersection of the palatal plane (PP, 4-24) with the occlusal plane (OP, 23-11).

PP-UOP - Angle formed by the intersection of the palatal plane (PP, 4-24) with the upper occlusal plane (UOP, 22-10).

PP-LOP - Angle formed by the intersection of the palatal plane (PP, 4-24) with the lower occlusal plane (LOP, 21-12).

PP-FOP - Angle formed by the intersection of the palatal plane (PP, 4-24) with the functional occlusal plane (FOP, 23-20).

ULD (Unit length difference) - Mandibular unit length (MdUL, 32-33) minus maxillary unit length (MxUL, 32-6).

VI Dentoalveolar

U.I-SN - Inferior posterior angle formed by the intersection of a line drawn through the long axis of the upper incisor (10-9) with the anterior cranial base (SN, I-2).

U.1-NA - The acute angle formed by the intersection of a line drawn through the long axis of the upper incisor (10-9) with a line joining "A" point to nasion $(7-2)$.

U.I to SN - Distance of a perpendicular from the upper central incisal 
edge (U.1, 10) to the anterior cranial base (SN, 1-2).

U.l to PP - Distance of a perpendicular from the upper central incisal edge (U.1, 10) to the palatal plane (PP, 4-24).

U.1 to NA - Distance of a perpendicular from the upper central incisal edge (U.1, 10) to a line joining "A" point with nasion (NA, 2-7).

U.1 to APg - Distance of a perpendicular from the upper central incisal edge $(\mathrm{U} .1,10)$ to a line joining "A" point with pogonion (APg, 7-16).

ADH (Anterior dental height) - Distance from anterior nasal spine (ANS, 4) to the upper central incisal edge (U.I, 10) along a perpendicular to the anterior cranial base (SN, 1-2).

U.6 to SN - Distance of a perpendicular from the upper molar mesiobuccal cusp tip (U.6, 22) to the anterior cranial base (SN, 1-2).

U.6 to PP - Distance of a perpendicular from the upper molar mesiobuccal cusp tip $(U .6,22)$ to the palatal plane (PP, 4-24).

U.6 to PP $\perp$ OP - Distance from the upper molar mesiobuccal cusp tip $(U .6,22)$ to the palatal plane ( $P P, 4-24)$ perpendicular to the occlusal plane (OP, 23-1I).

U. 6 to PP $\perp$ FOP - Distance from the upper molar mesiobuccal cusp tip $(\mathrm{U} .6,22)$ to the palatal plane (PP, 4-24) perpendicular to the functional occlusal plane (FOP, 23-20).

U.6 to PP $\perp$ UOP - Distance from the upper molar mesiobuccal cusp tip $(U .6,22)$ to the palatal plane (PP, 4-24) perpendicular to the upper occlusal plane (UOP, 22-10).

L.1-GoGn - Superior posterior angle formed by the intersection of a line drawn through the long axis of the lower incisor (12-14) with the mandibular plane (GoGn, 27-17).

L.1-GoMe - Superior posterior angle formed by the intersection of a 
line drawn through the long axis of the lower incisor (12-14) with the mandibular plane (GoMe, 27-18).

L.1-LBMe - Superior posterior angle formed by the intersection of a line drawn thgough the long axis of the lower incisor (12-14) with the mandibular plane (LBMe, 26-18).

L.1-NB - The acute angle formed by the intersection of a line drawn through the long axis of the lower incisor (12-14) with a line joining nasion with "B" point (NB, 2-15).

L.I to GoGn - Distance of a perpendicular from the lower central incisal edge (L.I, 12) to the mandibular plane (GoGn, 27-17).

L.I to GoMe - Distance of a perpendicular from the lower central incisal edge ( $\mathrm{L} .1,12$ ) to the mandibular plane (GoMe, 27-18).

L.I to LBMe - Distance of a perpendicular from the lower central incisal edge (L.I, 12) to the mandibular plane (LBMe, 26-18).

L.I to NB - Distance of a perpendicular from the lower central incisal edge (L.1, 12) to a line joining nasion to "B" point (NB, 2-15).

L.I to APg - Distance of a perpendicular from the lower central incisal edge ( $\mathrm{L} . \mathrm{I}, 12$ ) to a line joining "A" point with pogonion $(\mathrm{APg}, 7-16)$.

L.6 to GoGn - Distance of a perpendicular from the lower molar mesiobuccal cusp tip ( $2.6,21$ ) to the mandibular plane (GoGn, 27-17).

L. 6 to GoMe - Distance of a perpendicular from the lower molar mesiobuccal cusp tip (L.6, 21) to the mandibular plane (GoMe, 27-18).

L. 6 to LBMe - Distance of a perpendicular from the lower molar mesiobuccal cusp tip $(\mathrm{L} .6,21$ ) to the mandibular plane (LBMe, 26-18).

U.l to L.I - Posterior angle formed by the intersection of lines drawn through the long axis of the upper central incisor (9-10) and the long axis of the lower central incisor (12-14). 
VII Vertical Proportions

TAFH (Total anterior face height) - Distance between nasion ( $\mathrm{Na}, 2$ ) and menton (Me, 18).

UAFH (Upper anterior face height) - Distance between nasion ( $\mathrm{Na}, 2$ ) and a point obtained by projecting a perpendicular from the anterior nasal spine (ANS, 4) to the nasion menton line (2-18).

LAFH (Lower anterior face height) - Distance between a point obtained by projecting a perpendicular from anterior nasal spine (ANS, 4) to the nasion menton line and menton (Me, 18).

PFH (Posterior face height) - Distance from gonion to sella (Go-S, 27 $1-2)$.

PFH (Posterior face height) - Distance of a perpendicular from gonion to the anterior cranial base (Go $\perp$ SN, $27 \perp 1-2$ ).

Ramus Ht. - Distance between the superior aspect of the condyle (31) and gonion (Go, 27) measured perpendicular to the anterior cranial base (SN, 1-2).

UAFH - Ratio of upper anterior face height to lower anterior face height. 
Results of Students " $t$ " test between Group 1 - Overbite $\geq 0$ ( N.O.B. Grp. ) and Group 2 - Overbite <0 ( O. B. Grp. ) for the variables of tongue volume, cephalometric measurements and model measurements.

Table 11. Means, standard errors and level of significance between Group 1 - Overbite $\geq 0$ ( N. O. B. Grp. ) and Group 2 overbite $<0$ ( 0 . B. Grp. ) fcr the variable tongue volume

\begin{tabular}{ccccc}
\hline Variable & Grp. & Mean & Standard Error & Significance \\
\hline & 1 & 86.0348 & 0.685 & \\
$\frac{\text { Tongue Volume }}{\text { Oral Volume }}$ & 2 & 90.9100 & 0.530 & 0.005 \\
\hline
\end{tabular}


Table 12. Means, standard errors and levels of significance between Group 1 - Overbite $\geq 0$ ( N. O. B. Grp. ) and Group 2 Overbite $<0$ ( o. B. Grp. ) for cephalometric variables. Variable Grp. Mean Standard Error

I Intermaxillary Ht., Lth. and Area, Tongue and Hyoid

$\begin{array}{lllll}\text { Ant. I. M. Ht. } & 1 & 5.7874 & 0.073 & 0.003 \\ & 2 & 6.4026 & 0.155 & \\ \text { Post. I. M. Ht. } & 1 & 2.8524 & 0.098 & 0.190 \\ & 2 & 2.5150 & 0.204 & \\ \text { I. M. Ith. } & 1 & 7.2497 & 0.083 & 0.190 \\ & 2 & 7.1346 & 0.158 & \\ \text { I. M. Area } & 1 & 31.3624 & 0.722 & 0.798 \\ & 2 & 31.8350 & 1.316 & \\ \text { Vert. pos. of hyoid } & 1 & 6.2472 & 0.110 & 0.407 \\ & 2 & 6.4838 & 0.223 & \\ \text { Hor. pos. of tongue } & 1 & 28.2202 & 1.229 & 0.147\end{array}$

II Cranial Base

$\begin{array}{lrrrr}\text { SNBa } & 1 & 130.8307 & 0.830 & 0.860 \\ & 2 & 134.5570 & 1.499 & \\ \text { SN (mm.) } & 1 & 6.6701 & 0.057 & 0.172 \\ & 2 & 6.4714 & 0.070 & \\ \mathrm{SBa}(\mathrm{mm} .) & 1 & 4.3268 & 0.050 & 0.103\end{array}$

III Maxilla to Cranium

$\begin{array}{lrrrr} & 1 & 5.5067 & 0.653 & 0.046 \\ & 2 & 9.0132 & 1.439 & \\ \text { SNA } & 1 & 82.0951 & 0.707 & 0.010\end{array}$


159

\begin{tabular}{|c|c|c|c|c|}
\hline Variable & Grp. & Mean & Standard Error & Significance \\
\hline $\mathrm{NA}-\mathrm{FH}$ & $\frac{1}{2}$ & $\begin{array}{l}87.6019 \\
86.2490\end{array}$ & $\begin{array}{l}0.670 \\
0.497\end{array}$ & 0.417 \\
\hline Angle of Convexity & $\begin{array}{l}1 \\
2\end{array}$ & $\begin{array}{l}173.7192 \\
173.0356\end{array}$ & $\begin{array}{l}1.384 \\
2.874\end{array}$ & 0.849 \\
\hline $\mathrm{SN}-\mathrm{OP}$ & $\frac{1}{2}$ & $\begin{array}{l}14.5902 \\
20.6510\end{array}$ & $\begin{array}{l}0.864 \\
1.703\end{array}$ & 0.010 \\
\hline SN-UOP & $\begin{array}{l}1 \\
2\end{array}$ & $\begin{array}{l}17.0285 \\
18.3086\end{array}$ & $\begin{array}{l}0.882 \\
1.385\end{array}$ & 0.567 \\
\hline SN-FOP & $\begin{array}{l}1 \\
2\end{array}$ & $\begin{array}{l}15.8581 \\
25.8334\end{array}$ & $\begin{array}{l}0.957 \\
1.742\end{array}$ & 0.000 \\
\hline $\mathrm{SN}-\mathrm{PP}$ & $\begin{array}{l}1 \\
2\end{array}$ & $\begin{array}{l}6.6250 \\
9.3646\end{array}$ & $\begin{array}{l}0.696 \\
0.884\end{array}$ & 0.123 \\
\hline ANS-PNS (mm.) & $\begin{array}{l}1 \\
2\end{array}$ & $\begin{array}{l}5.3560 \\
5.1276\end{array}$ & $\begin{array}{l}0.052 \\
0.098\end{array}$ & 0.092 \\
\hline MxUL (m. $)$ & $\begin{array}{l}1 \\
2\end{array}$ & $\begin{array}{l}8.6417 \\
8.2510\end{array}$ & $\begin{array}{l}0.094 \\
0.125\end{array}$ & 0.104 \\
\hline IV Mandible to Cra & nium & & & \\
\hline Facial Angle & $\begin{array}{l}1 \\
2\end{array}$ & $\begin{array}{l}84.6221 \\
82.6026\end{array}$ & $\begin{array}{l}0.722 \\
1.579\end{array}$ & 0.286 \\
\hline SNB & $\frac{1}{2}$ & $\begin{array}{l}77.9154 \\
73.3718\end{array}$ & $\begin{array}{l}0.760 \\
2.130\end{array}$ & 0.032 \\
\hline SN-IOP & $\begin{array}{l}1 \\
2\end{array}$ & $\begin{array}{r}8.5511 \\
21.7864\end{array}$ & $\begin{array}{l}1.055 \\
2.557\end{array}$ & 0.000 \\
\hline$Y$ axis & $\begin{array}{l}1 \\
2\end{array}$ & $\begin{array}{l}61.6534 \\
66.2010\end{array}$ & $\begin{array}{l}0.700 \\
2.090\end{array}$ & 0.022 \\
\hline SN-GOGn & $\begin{array}{l}1 \\
2\end{array}$ & $\begin{array}{l}31.0069 \\
43.9668\end{array}$ & $\begin{array}{l}1.136 \\
3.772\end{array}$ & 0.000 \\
\hline $\mathrm{SN}-\mathrm{GOMe}$ & $\begin{array}{l}1 \\
2\end{array}$ & $\begin{array}{l}32.7990 \\
45.8888\end{array}$ & $\begin{array}{l}1.130 \\
3.646\end{array}$ & 0.000 \\
\hline $\mathrm{SN}$-IBMe & $\begin{array}{l}1 \\
2\end{array}$ & $\begin{array}{l}31.5709 \\
45.6838\end{array}$ & $\begin{array}{l}1.235 \\
3.842\end{array}$ & 0.000 \\
\hline $\begin{array}{l}\text { Gonial Angle (sup. } \\
\text { condyle-GoGn) }\end{array}$ & $\frac{1}{2}$ & $\begin{array}{l}121.5576 \\
129.4758\end{array}$ & $\begin{array}{l}1.026 \\
3.259\end{array}$ & 0.008 \\
\hline
\end{tabular}


100

\begin{tabular}{|c|c|c|c|c|}
\hline Variable & Grp. & Mean & Standard Error & Significance \\
\hline Corpus Lth. & $\begin{array}{c}\text { (GO-Gn, } \\
\text { num.) }\end{array}$ & $\begin{array}{l}6.7176 \\
6.4888\end{array}$ & $\begin{array}{l}0.107 \\
0.197\end{array}$ & 0.406 \\
\hline MdUL (mm.) & $\begin{array}{l}1 \\
2\end{array}$ & $\begin{array}{l}10.8785 \\
11.0196\end{array}$ & $\begin{array}{l}0.134 \\
0.158\end{array}$ & 0.674 \\
\hline \multicolumn{5}{|c|}{ V Mandible to Maxilla } \\
\hline ANB & $\begin{array}{l}1 \\
2\end{array}$ & $\begin{array}{l}4.1794 \\
3.8640\end{array}$ & $\begin{array}{l}0.566 \\
1.291\end{array}$ & 0.831 \\
\hline $\mathrm{OP}-\mathrm{GOG}$ & $\frac{1}{2}$ & $\begin{array}{l}16.4164 \\
23.3156\end{array}$ & $\begin{array}{l}0.730 \\
2.301\end{array}$ & 0.002 \\
\hline OP-GOMe & $\begin{array}{l}1 \\
2\end{array}$ & $\begin{array}{l}18.2086 \\
25.2374\end{array}$ & $\begin{array}{l}0.717 \\
2.209\end{array}$ & 0.001 \\
\hline OP-IBMe & $\frac{1}{2}$ & $\begin{array}{l}16.9808 \\
25.0328\end{array}$ & $\begin{array}{l}0.828 \\
2.361\end{array}$ & 0.001 \\
\hline UOP-GOGn & $\frac{1}{2}$ & $\begin{array}{l}13.9780 \\
25.6574\end{array}$ & $\begin{array}{l}0.897 \\
2.409\end{array}$ & 0.000 \\
\hline WOP-GOMe & $\begin{array}{l}1 \\
2\end{array}$ & $\begin{array}{l}15.7704 \\
27.5796\end{array}$ & $\begin{array}{l}0.877 \\
2.293\end{array}$ & 0.000 \\
\hline UOP-IBMe & $\begin{array}{l}1 \\
2\end{array}$ & $\begin{array}{l}14.5422 \\
29.3748\end{array}$ & $\begin{array}{l}0.980 \\
2.488\end{array}$ & 0.000 \\
\hline LOP-GOGn & $\begin{array}{l}1 \\
2\end{array}$ & $\begin{array}{l}22.4555 \\
22.1802\end{array}$ & $\begin{array}{l}0.862 \\
2.017\end{array}$ & 0.903 \\
\hline IOP-GOMe & $\begin{array}{l}1 \\
2\end{array}$ & $\begin{array}{l}24.2480 \\
24.1022\end{array}$ & $\begin{array}{l}0.858 \\
2.005\end{array}$ & 0.948 \\
\hline LOP-IBMe & $\begin{array}{l}1 \\
2\end{array}$ & $\begin{array}{l}23.0196 \\
23.8974\end{array}$ & $\begin{array}{l}0.949 \\
2.071\end{array}$ & 0.722 \\
\hline FOP-GoGn & $\begin{array}{l}1 \\
2\end{array}$ & $\begin{array}{l}15.1485 \\
18.1334\end{array}$ & $\begin{array}{l}0.741 \\
2.421\end{array}$ & 0.151 \\
\hline FOP-GOMe & $\begin{array}{l}1 \\
2\end{array}$ & $\begin{array}{l}16.9407 \\
20.0550\end{array}$ & $\begin{array}{l}0.719 \\
2.301\end{array}$ & 0.122 \\
\hline FOP-LBMe & $\begin{array}{l}1 \\
2\end{array}$ & $\begin{array}{l}15.7127 \\
19.8502\end{array}$ & $\begin{array}{l}0.781 \\
2.446\end{array}$ & 0.060 \\
\hline$P P-G O G n$ & $\begin{array}{l}1 \\
2\end{array}$ & $\begin{array}{l}24.3816 \\
34.6024\end{array}$ & $\begin{array}{l}1.087 \\
3.222\end{array}$ & 0.001 \\
\hline
\end{tabular}




\begin{tabular}{lcrcc}
\hline Variable & GrP. & Mean & Standard Frror & Significance \\
\hline \multirow{2}{*}{ PP-GOMe } & 1 & 26.1710 & 1.061 & \\
& 2 & 36.5240 & 3.126 & 0.001 \\
PP-IBMe & 1 & 24.9459 & 1.182 & \\
& 2 & 36.3192 & 3.278 & 0.001 \\
PP-OP & 1 & 7.9651 & 0.605 & \\
& 2 & 11.2864 & 1.345 & 0.041 \\
PP-UOP & 1 & 10.4034 & 0.587 & \\
& 2 & 8.9446 & 1.029 & 0.334 \\
PP-IOP & 1 & 1.9260 & 0.860 & \\
& 2 & 12.4220 & 2.080 & 0.000 \\
PP-FOP & 1 & 9.2331 & 0.788 & \\
& 2 & 16.4688 & 1.492 & 0.001 \\
ULD & 1 & 2.2368 & 0.110 & \\
& 2 & 2.7686 & 0.122 & 0.059
\end{tabular}

VI Dentoalveolar

\begin{tabular}{|c|c|c|c|c|}
\hline $\mathrm{U} .1-\mathrm{SN}$ & $\begin{array}{l}1 \\
2\end{array}$ & $\begin{array}{l}106.1441 \\
107.4654\end{array}$ & $\begin{array}{l}1.344 \\
2.387\end{array}$ & 0.700 \\
\hline $\mathrm{U} .1-\mathrm{NA}$ & $\begin{array}{l}1 \\
2\end{array}$ & $\begin{array}{l}24.0491 \\
30.2294\end{array}$ & $\begin{array}{l}1.355 \\
2.459\end{array}$ & 0.082 \\
\hline U.I to SN (mm.) & $\begin{array}{l}1 \\
2\end{array}$ & $\begin{array}{l}7.4222 \\
7.6744\end{array}$ & $\begin{array}{l}0.062 \\
0.212\end{array}$ & 0.153 \\
\hline U.1 to PP $\left(\mathrm{mm}_{0}\right)$ & $\begin{array}{l}1 \\
2\end{array}$ & $\begin{array}{l}2.6336 \\
2.8278\end{array}$ & $\begin{array}{l}0.038 \\
0.127\end{array}$ & 0.074 \\
\hline U.I to NA (mm.) & $\frac{1}{2}$ & $\begin{array}{l}0.4087 \\
0.6882\end{array}$ & $\begin{array}{l}0.052 \\
0.070\end{array}$ & 0.038 \\
\hline U. 1 to APg (mm.) & $\frac{1}{2}$ & $\begin{array}{l}0.6322 \\
0.9658\end{array}$ & $\begin{array}{l}0.055 \\
0.133\end{array}$ & 0.027 \\
\hline $\mathrm{ADH}$ (mm.) & $\frac{1}{2}$ & $\begin{array}{l}2.5859 \\
2.7736\end{array}$ & $\begin{array}{l}0.039 \\
0.116\end{array}$ & 0.085 \\
\hline U. 6 to $\mathrm{SN}\left(\mathrm{mm}_{0}\right)$ & $\frac{1}{2}$ & $\begin{array}{l}6.5312 \\
6.6592\end{array}$ & $\begin{array}{l}0.059 \\
0.203\end{array}$ & 0.442 \\
\hline $\mathrm{U} .6$ to $\mathrm{PP}(\mathrm{mm})$. & $\begin{array}{l}1 \\
2\end{array}$ & $\begin{array}{l}2.0814 \\
2.3194\end{array}$ & $\begin{array}{l}0.037 \\
0.083\end{array}$ & 0.019 \\
\hline
\end{tabular}


162

\begin{tabular}{|c|c|c|c|c|}
\hline Variable & Grp. & Mean & Standard Error & Significance \\
\hline $\mathrm{U} .6$ to $\mathrm{PP} \perp \mathrm{OP}\left(\mathrm{mm}_{\bullet}\right)$ & $\begin{array}{l}1 \\
2\end{array}$ & $\begin{array}{l}2.1047 \\
2.3678\end{array}$ & $\begin{array}{l}0.037 \\
0.086\end{array}$ & 0.010 \\
\hline $\mathrm{U} .6$ to $\mathrm{PP} \perp \mathrm{FOP}(\mathrm{mm})$. & $\frac{1}{2}$ & $\begin{array}{l}2.1140 \\
2.4206\end{array}$ & $\begin{array}{l}0.037 \\
0.088\end{array}$ & 0.003 \\
\hline $\mathrm{U} .6$ to $\mathrm{PP} \perp \mathrm{UOP}(\mathrm{mm})$. & $\begin{array}{l}1 \\
2\end{array}$ & $\begin{array}{l}1.5000 \\
1.6426\end{array}$ & $\begin{array}{l}0.025 \\
0.068\end{array}$ & 0.038 \\
\hline L.1-GoGn & $\begin{array}{l}1 \\
2\end{array}$ & $\begin{array}{l}95.0601 \\
89.6508\end{array}$ & $\begin{array}{l}1.315 \\
2.624\end{array}$ & 0.117 \\
\hline L. 1-GoMe & $\begin{array}{l}1 \\
2\end{array}$ & $\begin{array}{l}93.2676 \\
87.7288\end{array}$ & $\begin{array}{l}1.302 \\
2.678\end{array}$ & 0.107 \\
\hline L. 1-LBMe & $\begin{array}{l}1 \\
2\end{array}$ & $\begin{array}{l}94.4959 \\
87.9336\end{array}$ & $\begin{array}{l}1.322 \\
2.724\end{array}$ & 0.062 \\
\hline L. I-NB & $\begin{array}{l}1 \\
2\end{array}$ & $\begin{array}{l}23.9825 \\
26.9900\end{array}$ & $\begin{array}{l}1.083 \\
2.856\end{array}$ & 0.301 \\
\hline I. 1 to GoGn (nm.) & $\begin{array}{l}1 \\
2\end{array}$ & $\begin{array}{l}3.6480 \\
3.7212\end{array}$ & $\begin{array}{l}0.046 \\
0.169\end{array}$ & 0.577 \\
\hline L.I to GoMe (mm.) & $\begin{array}{l}1 \\
2\end{array}$ & $\begin{array}{l}3.8333 \\
3.9050\end{array}$ & $\begin{array}{l}0.047 \\
0.165\end{array}$ & 0.590 \\
\hline L. I to IBMe (mm.) & $\begin{array}{l}1 \\
2\end{array}$ & $\begin{array}{l}3.8419 \\
3.9072\end{array}$ & $\begin{array}{l}0.046 \\
0.165\end{array}$ & 0.616 \\
\hline L. I to NB (mm.) & $\begin{array}{l}1 \\
2\end{array}$ & $\begin{array}{l}0.4222 \\
0.5998\end{array}$ & $\begin{array}{l}0.040 \\
0.173\end{array}$ & 0.143 \\
\hline L.1 to APg (mm.) & $\begin{array}{l}1 \\
2\end{array}$ & $\begin{array}{l}0.0829 \\
0.3614\end{array}$ & $\begin{array}{l}0.040 \\
0.120\end{array}$ & 0.015 \\
\hline L. 6 to GoGn (mm.) & $\begin{array}{l}1 \\
2\end{array}$ & $\begin{array}{l}2.6918 \\
2.8322\end{array}$ & $\begin{array}{l}0.046 \\
0.112\end{array}$ & 0.248 \\
\hline L. 6 to GoMe (mm.) & $\begin{array}{l}1 \\
2\end{array}$ & $\begin{array}{l}2.8054 \\
2.9436\end{array}$ & $\begin{array}{l}0.048 \\
0.113\end{array}$ & 0.274 \\
\hline L. 6 to LBMe (mm.) & $\begin{array}{l}1 \\
2\end{array}$ & $\begin{array}{l}2.8622 \\
2.9534\end{array}$ & $\begin{array}{l}0.048 \\
0.119\end{array}$ & 0.476 \\
\hline U.1 to I.I & $\frac{1}{2}$ & $\begin{array}{l}127.7885 \\
118.9166\end{array}$ & $\begin{array}{l}1.737 \\
4.615\end{array}$ & 0.062 \\
\hline
\end{tabular}


163

\begin{tabular}{|c|c|c|c|c|}
\hline Variable & Grp. & Mean & Standard Error & Significance \\
\hline \multicolumn{5}{|c|}{ VII Vertical Proportions } \\
\hline TAFH (mm.) & $\begin{array}{l}1 \\
2\end{array}$ & $\begin{array}{l}10.7371 \\
11.5664\end{array}$ & $\begin{array}{l}0.096 \\
0.281\end{array}$ & 0.003 \\
\hline UAF'H (mm.) & $\begin{array}{l}1 \\
2\end{array}$ & $\begin{array}{l}4.6932 \\
4.7632\end{array}$ & $\begin{array}{l}0.050 \\
0.101\end{array}$ & 0.585 \\
\hline IAFH (mm.) & $\begin{array}{l}1 \\
2\end{array}$ & $\begin{array}{l}6.0440 \\
6.8032\end{array}$ & $\begin{array}{l}0.080 \\
0.239\end{array}$ & 0.001 \\
\hline PFH (Go-S,mm.) & $\begin{array}{l}1 \\
2\end{array}$ & $\begin{array}{l}7.1444 \\
6.7854\end{array}$ & $\begin{array}{l}0.105 \\
0.242\end{array}$ & 0.198 \\
\hline $\mathrm{PFH} \quad(\mathrm{GO} \perp \mathrm{SN}, \mathrm{mm})$. & $\begin{array}{l}1 \\
2\end{array}$ & $\begin{array}{l}6.9989 \\
6.5354\end{array}$ & $\begin{array}{l}0.109 \\
0.257\end{array}$ & 0.111 \\
\hline 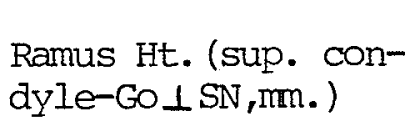 & $\begin{array}{l}1 \\
2\end{array}$ & $\begin{array}{l}5.3883 \\
5.3566\end{array}$ & $\begin{array}{l}0.098 \\
0.187\end{array}$ & 0.899 \\
\hline$\frac{\mathrm{UAFH}}{\mathrm{IAFH}}$ & $\begin{array}{l}1 \\
2\end{array}$ & $\begin{array}{l}0.7802 \\
0.7032\end{array}$ & $\begin{array}{l}0.013 \\
0.025\end{array}$ & 0.030 \\
\hline
\end{tabular}


Table 13. Means, standard errors and levels of significance between Group 1 - Overbite $\geq 0$ ( N. O. B. Grp. ) and Group 2 Overbite $<0$ ( O. B. Grp. ) for model variables.

\begin{tabular}{|c|c|c|c|c|}
\hline Variable & Grp. & Mean & Standard Error & Significance \\
\hline overjet & $\begin{array}{l}1 \\
2\end{array}$ & $\begin{array}{l}5.4519 \\
5.0830\end{array}$ & $\begin{array}{l}0.560 \\
0.658\end{array}$ & 0.792 \\
\hline Overbite & $\begin{array}{l}1 \\
2\end{array}$ & $\begin{array}{r}4.1310 \\
-1.8600\end{array}$ & $\begin{array}{l}0.341 \\
0.590\end{array}$ & 0.000 \\
\hline Arch Lth. -U & $\begin{array}{l}1 \\
2\end{array}$ & $\begin{array}{l}28.8002 \\
29.3720\end{array}$ & $\begin{array}{l}0.561 \\
0.902\end{array}$ & 0.688 \\
\hline Arch Lth. -I & $\frac{1}{2}$ & $\begin{array}{l}23.6431 \\
21.9180\end{array}$ & $\begin{array}{l}0.454 \\
0.896\end{array}$ & 0.147 \\
\hline Intercanine Wth. - L & $\frac{1}{2}$ & $\begin{array}{l}26.5536 \\
25.8740\end{array}$ & $\begin{array}{l}0.467 \\
0.873\end{array}$ & 0.571 \\
\hline Intermolar wth. -U & $\begin{array}{l}1 \\
2\end{array}$ & $\begin{array}{l}38.1131 \\
38.3230\end{array}$ & $\begin{array}{l}0.649 \\
2.170\end{array}$ & 0.907 \\
\hline Intermolar wth. - I & $\begin{array}{l}1 \\
2\end{array}$ & $\begin{array}{l}33.8122 \\
33.2690\end{array}$ & $\begin{array}{l}0.638 \\
1.610\end{array}$ & 0.748 \\
\hline Arch Circum.-U & $\begin{array}{l}1 \\
2\end{array}$ & $\begin{array}{l}78.5903 \\
78.1610\end{array}$ & $\begin{array}{l}1.108 \\
2.829\end{array}$ & 0.884 \\
\hline Arch Circum.-I & $\begin{array}{l}1 \\
2\end{array}$ & $\begin{array}{l}67.3924 \\
64.4790\end{array}$ & $\begin{array}{l}0.883 \\
2.244\end{array}$ & 0.218 \\
\hline Palatal Vault Ht. & $\begin{array}{l}1 \\
2\end{array}$ & $\begin{array}{l}16.2193 \\
17.1540\end{array}$ & $\begin{array}{l}0.411 \\
1.224\end{array}$ & 0.405 \\
\hline
\end{tabular}




\section{Appendix VIII}

Table 14. Tongue Volume, Oral Volume and $\frac{\text { Tongue Volume }}{\text { Oral Volume }}$. 100\% for Group 1 - Overbite $\geq 0$ ( N. O. B. Grp. ) and Group 2 - Overbite $<0$ ( O. B. Grp. ).

\begin{tabular}{|c|c|c|c|c|}
\hline Subject & Grp. & $\begin{array}{c}\text { Tongue Jolume } \\
\left(\mathrm{cm}^{3}\right)\end{array}$ & $\begin{array}{l}\text { Oral Vqlume } \\
\left(\mathrm{cm}^{3}\right)\end{array}$ & $\begin{array}{c}\text { Tongue Volume } \\
\text { Oral Volume } \\
(100 \%)\end{array}$ \\
\hline C. Ad. & 1 & 49.34 & 56.20 & 87.79 \\
\hline B. Al. & 1 & 55.70 & 66.25 & 86.79 \\
\hline B. Bo. & 1 & 84.50 & 95.75 & 88.25 \\
\hline C. Ca. & 1 & 60.00 & 69.25 & 86.64 \\
\hline D. Ch. & 1 & 60.95 & 72.23 & 84.38 \\
\hline M. De. & 1 & 61.00 & 75.00 & 81.33 \\
\hline D. Fu. & 1 & 48.42 & 56.82 & 85.22 \\
\hline D. Ge. & 2 & 56.83 & 62.99 & 89.51 \\
\hline S. Gr. & 1 & 59.12 & 66.39 & 89.05 \\
\hline R. Had. & 1 & 45.00 & 53.00 & 84.91 \\
\hline M. Han. & 1 & 50.00 & 59.50 & 84.03 \\
\hline Y. He. & 1 & 60.13 & 67.32 & 89.32 \\
\hline W. Jac. & 2 & 62.52 & 67.85 & 92.14 \\
\hline I. Jan. & 1 & 47.25 & 63.50 & 74.41 \\
\hline J. Ki. & 1 & 66.97 & 75.46 & 88.75 \\
\hline P. Ii. & 2 & 55.10 & 60.05 & 91.76 \\
\hline K. Mat. & 1 & 54.75 & 65.25 & 83.90 \\
\hline D. May & 1 & 53.25 & 64.00 & 83.20 \\
\hline N. Maz. & 1 & 59.73 & 68.35 & 87.39 \\
\hline T. MO & 1 & 76.19 & 85.94 & 88.65 \\
\hline C. Pi. & 2 & 72.49 & 79.36 & 91.34 \\
\hline C. Po. & 1 & 62.38 & 69.88 & 89.27 \\
\hline T. Qu. & 1 & 74.50 & 82.75 & 90.03 \\
\hline R. Ri. & 1 & 68.88 & 78.88 & 87.32 \\
\hline B. Ro. & 1 & 70.00 & 78.00 & 89.74 \\
\hline D. Sc. & 1 & 46.50 & 57.50 & 80.87 \\
\hline
\end{tabular}




\begin{tabular}{llccc}
\hline Subject & Grp. & $\begin{array}{c}\text { Tongue Vqlume } \\
\left(\mathrm{cm}^{3}\right)\end{array}$ & $\begin{array}{c}\text { Oral Volume } \\
\left(\mathrm{cm}^{3}\right)\end{array}$ & $\begin{array}{c}\text { Tongue Volume } \\
\text { Oral 188 lum })\end{array}$ \\
\hline C. Ter. & 2 & 59.50 & 66.26 & 89.80 \\
C. Tes. & 1 & 53.00 & 63.00 & 84.13 \\
D. Th. & 1 & 71.61 & 82.97 & 86.31 \\
M. To. & 1 & 42.63 & 51.63 & 82.57 \\
D. Wo. & 1 & 52.57 & 58.63 & 89.66 \\
L. Yo. & 1 & 63.73 & 71.58 & 89.03 \\
& & & & \\
\hline
\end{tabular}


Appendix IX

Discussion of Cephalometric Variables Between Group $1=$ Overbite $\underline{0}$

$(\underline{N} \cdot \underline{0} \cdot \underline{B} \cdot \underline{\text { Group })}$ and Group $\underline{2}=$ Overbite $\underline{0}(\underline{0} \cdot \underline{B} \cdot \underline{\text { Group }})$

A more detailed discussion of the cephalometric results reported in this study (Table 12, Appendix VII) for open-bite (0.B.) subjects as opposed to nonopen-bite (N.O.B.) subjects is presented here, while a shorter summarized version appears in the "Discussion". The openbite sample of this study was compared with the open-bite samples of other investigators (Subtelny and Sakuda, 1964; Isaacson et al., 1971; Nahoum, 1971, 1975; Nahoum et al., 1972; Lowe, 1980).

Evaluating the cranial base in this study, no difference was found between the O.B. and N.O.B. groups in the angulation of the cranial base or in the length of either posterior or anterior cranial base, although Subtelny and Sakuda (1964) found the posterior cranial base $(\mathrm{SBa})$ to be significantly shorter $(p<0.05)$ in $0 . B$. subjects.

Although the skeletal maxilla was comparable in size in the two groups in this study (ANS-PNS, MXUL), the SNA angle was found to be smaller at the one percent level of significance in the $0 . \mathrm{B}$. group, indicating that the base of the maxilla is retropositioned in relation to the anterior cranial base in $O . B$. subjects. This finding is in agreement with Subtelny and Sakuda (1964). Isaacson et al. (1971) also found decreased SNA angles in their high mandibular plane group, which also exhibited anterior O.B. malocclusion. However Lowe (1980) found SNA angles to be within normal limits for his $0 . B$. sample.

In $0 . B$. subjects, a distortion of the maxilla is often evident, 
as the premaxilla seems to tip up anteriorly toward the base of the skull giving the impression that the premaxillary area did not grow vertically. Although Nahoum (1971) did find the SN-PP angle to be significantly smaller $(p<0.001)$, Subtelny and Sakuda (1964), Lowe (1980) and this investigator found no significant difference in SN-PP between $0 . B$. and N.O.B. groups.

The commonly used occlusal plane (OP) is constructed by a line bisecting the vertical overlap of the first permanent molars and the permanent central incisors. Nahoum et al. (1972) have drawn two occlusal planes for $0 . B$. subjects by extending a line from the mesiobuccal cusp tips of the first permanent molars to the incisal edges of either the maxillary or mandibular central incisors (Upper occlusal plane - UOP; Lower occlusal plane - LOP). This investigator has used these planes plus an additional one, the functional occlusal plane (FOP), as defined by Vasiga (1972), which is constructed through the bisection of the vertical overlap of the first permanent molars and first and second bicuspids. In this study the SN-OP angle was found to be larger for the $0 . B$. group at the one percent level of significance which is in agreement with Subtelny and Sakuda (1964), Isaacson et al. (1971) and Lowe (1980). The SN-UOP angle showed little variation between the two groups studied in this investigation while SN-LOP was significantly greater $(p<0.01)$ for the $0 . B$. group. This is in agreement with Nahoum (1972). The SN-FOP angle is also significantly larger $(p<0.01)$ in the $0 . B$. sample studied here. Therefore steep occlusal plane angles were exhibited whether using OP, LOP or FOP. 
The SN-UOP angle was similar for O.B. and N.O.B. subjects.

Differences were found in the shape and position of the mandible between the two groups in this study. The SNB angle was significantly smaller $(p<0.05)$ in the $0 . B$. group indicating that the mandible was retruded relative to the cranial base. Subtelny and Sakuda (1964) also found decreased SNB angles at $p<0.001$. Isaacson et al. (1971) found decreased SNB angles in the high mandibular plane group. Lowe (1980) found no significant difference between his $0 . B$. and normal sample, although the SNB angles were smaller for 0. B. subjects.

Three different commonly used mandibular planes were examined in this study (LBMe, GoGn, GoMe) and all mandibular plane angles, using any of the three mandibular planes, were found to be significantly larger $(p<0.01)$ in the $0 . B$. group in this study. This is in agreement with Subtelny and Sakuda (1964), Nahoum (1971), Nahoum et al., (1972) and Lowe (1980), who also found significantly larger mandibular plane angles in $0 . B$. subjects. Although this investigator found no significant difference in corpus length (GoGn) or ramus height (superior aspect of the condyle to Go), the relationship of the body to the ramus of the mandible was found to be distorted as evidenced by larger gonial angles in $0 . B$. subjects at a significance level of less than one percent. Significantly increased gonial angles were also found by Subtelny and Sakuda (1964) and Nahoum (1972) for 0.B. subjects. In conjunction with increased mandibular plane angles and increased gonial angles, the $Y$ axis angle was also found to be significantly larger $(p<0.05)$ in this study's O.B. group, indicating a 
backward rotation of the mandible. Subtelny and Sakuda (1964) also found significantly larger $\mathrm{Y}$ axis angles in their 0.B. sample.

Examining the relationship of the mandible to the maxilla, this study found no significant difference in the ANB angle between $0 . B$. and N.O.B. subjects. Isaacson et al. (1971) found ANB angles to be similar for subjects with low, average or high mandibular plane angles. However both Subtelny and Sakuda (1964) and Lowe (1980) found significantly larger ANB angles in their 0.B. samples, indicative of greater spatial discrepancy of the mandible to the maxilla. This may be because their studies compared anterior open-bite subjects to normal occlusions, whereas in this study, the nonopen-bite subjects included Class I, Class II and Class III maloclusions.

Subtelny and Sakuda (1964), Nahoum (1971), Nahoum et al., (1972), Lowe (1980) and this investigator all found the PP-mandibular plane angles to be significantly larger $(p<0.01)$ in $0 . B$. subjects, indicating the divergence of the mandible from the maxilla. In addition, this study found both the OP-mandibular plane angle and UOP-mandibular plane angle to be significantly larger $(p<0.01)$ in $0 . B$. subjects, while no significant difference was found for the LOP-mandibular plane angle or FOP-mandibular plane angle between the two groups. This indicates that in $0 . B$. subjects, the mandible diverges from the maxilla (as represented by the $O P$ and UOP), whereas the LOP, being intimately related to the mandible, will show comparable angulations to the mandibular plane in both groups. The FOP-mandibular plane angle, although larger in $O . B$. subjects, was not significantly different 
from the N.O.B. group.

It has been thought that lack of development of the premaxillary region might be one factor in the etiology of anterior open-bite malocclusion. However, this study showed no significant difference between the O.B. and N.O.B. groups in the linear distances of the upper incisor from either SN or PP, indicating that vertical development of the premaxillary area is similar in both groups. On the other hand, this study found greater vertical development in the maxillary molar region of $0 . B$. subjects as indicated by significantly greater distances of $U .6$ to $P P(p<0.05), U .6$ to PP $\perp O P(p=0.01), U .6$ to PP $\perp$ FOP $(p<0.0 I)$ and U.6 to PP $\perp \operatorname{UOP}(p<0.05)$. The distance U.6 to $\mathrm{SN}$ was also larger in $\mathrm{O} . \mathrm{B}$. subjects but not significant. Subtelny and Sakuda (1964) found significantly greater vertical development of both the maxillary incisor and molar region (relative to $P P$ ), while Nahoum et al. (1972) found significant under-eruption of the maxillary incisor relative to $\mathrm{SN}$ but not to $\mathrm{PP}$, with maxillary molar development (relative to $\mathrm{SN}$ and $\mathrm{PP}$ ) similar to the control group.

Considering vertical development of the mandibular molar and incisor regions, this study found no significant difference between O.B. and N.O.B. subjects in the distances of L.I and L.6 to the mandibular plane. This agrees with Subtelny and Sakuda (1964), Nahoum (1971), Nahoum et al. (1972) except that Nahoum et al. (1972) and Nahoum (1975) found the dentoalveolar height of the mandibular first molar to be significantly decreased in $0 . B$. subjects.

The angulation of the upper incisor relative to $\mathrm{SN}$ and $\mathrm{NA}$, angu- 
lation of the lower incisor relative to the mandibular plane and NB and the interincisal angle were found to be not significantly different for the two groups in this study.

In this study, O.B. subjects were found to have significantly greater $(p<0.01)$ TAFH and LAFH, while UAFH was similar to the N.O.B. group. This resulted in a ratio of $\frac{\text { UAFH }}{\mathrm{LAFH}}$ which was smaller in $0 . \mathrm{B}$. subjects at a significance level of less than one percent. The results of Subtelny and Sakuda (1964) agree with the above and were found to be significant $(p<0.001)$. Isaacson et al. (1971) also found increased TAFH and LAFH in subjects with high mandibular plane angles. Nahoum (1971) found that while LAFH was significantly greater in $0 . B$. subjects, UAFH was significantly shorter and TAFH was similar or slightly longer compared to the control sample. Nahoum's ratio of $\frac{\mathrm{UAFH}}{\mathrm{I} A \mathrm{AFH}}$ was significantly smaller $(\mathrm{p}<0.001)$ in $0 . \mathrm{B}$. subjects. Lowe (1980), on the other hand, found no significant difference between his O.B. and control sample for UAFH, LAFH and TAFH. Lowe feels that his findings, in contrast to others, may reflect the fact the class I skeletal open-bites are composed of two subgroups, with one subgroup exhibiting considerably longer LAFH and TAFH.

Nahoum et al. (1972) have found $\mathrm{PFH}$ (Go-S) to be significantly shorter in $0 . B$. subjects. While in this study there was no significant difference between the two groups, the 0.B. group did show a trend towards decreased PFH. Isaacson et al. (1971) found PFH (Go $\perp$ SN) was decreased in subjects with high mandibular plane angles. In agreement with larger LAFH in O.B. subjects, Ant. I.M. Ht. 
(anterior intermaxillary height), a measurement devised by Vig and Cohen (1974), was also found to be significantly larger $(p<0.01)$ in O.B. subjects in this study. While Post. I.M. Ht. (posterior intermaxillary height) was not significantly different between the two groups, the O.B. group did show a tendency to smaller values for this measurement. Also I.M. Lth. (intermaxillary length) showed a tendency to be smaller in $0 . B$. subjects, but not at a level of significance, indicating that anteroposteriorly there is decreased space for the tongue within the oral cavity and oral pharynx. 